\title{
Like Thermodynamics before Boltzmann. On the Emergence of Einstein's Distinction between Constructive and Principle Theories
}

\author{
Marco Giovanelli \\ Forum Scientiarum - Universität Tübingen, \\ Doblerstrasse 3372074 Tübingen, Germany \\ marco.giovanelli@uni-tuebingen.de
}

How must the laws of nature be constructed in order to rule out the possibility of bringing about perpetual motion?

Einstein to Solovine, undated

\begin{abstract}
In a 1919 article for the Times of London, Einstein declared the relativity theory to be a 'principle theory', like thermodynamics, rather than a 'constructive theory,' like the kinetic theory of gases. The present paper attempts to trace back the prehistory of this famous distinction through a systematic overview of Einstein's repeated use of the relativity theory/thermodynamics analysis after 1905. Einstein initially used the comparison to address a specific objection. In his 1905 relativity paper he had determined the velocity-dependence of the electron's mass by adapting Newton's particle dynamics to the relativity principle. However, according to many, this result was not admissible without making some assumption about the structure of the electron. Einstein replied that the relativity theory is similar to thermodynamics. Unlike the usual physical theories, it does not directly try to construct models of specific physical systems; it provides empirically motivated and mathematically formulated criteria for the acceptability of such theories. New theories can be obtained by modifying existing theories valid in limiting case so that they comply with such criteria. Einstein progressively transformed this line of the defense into a positive heuristics. Instead of directly searching for new theories, it is often more effective to search for conditions which constraint the number of possible theories. The paper argues that the latter was the strategy that led Einstein to most of his major successes. The constructive/principle theories opposition should be considered not only as abstract classification of theories, but also as Einstein's attempt to formulate a sort of 'logic of discovery.' The paper argues that most of Einstein's scientific successes were obtained by following the principle strategy. Most of his failures happened when he was forced to fall back to the constructive strategy.
\end{abstract}

Keywords: Albert Einstein • Constructive theories $\bullet$ Principle theories $\bullet$ Relativity theory $\bullet$ Electron theories $\bullet$ Scientific discovery

\section{Introduction}

Toward the end of 1919, in a two-column contribution for the Times of London, Einstein (1919d) famously declared relativity theory to be a 'principle theory,' like thermodynamics, rather than a 'constructive theory,' like the kinetic theory of gases. Over the last decade, this distinction has attracted considerable attention in both the historically and the theoretically-oriented scholarship in the philosophy of physics. On occasion of the centenary of the publication of this brief but iconic article, it might be a good opportunity to try to reconstruct its history and assess its philosophical meaning. As it turns out, its popularity in today scholarship has somewhat hindered the appreciation of its core message. Contemporary philosophers of physics have often believed to have found in Einstein's sparingly phrased remarks support for their philosophical agenda. However, Einstein's concerns by writing the article bore probably only a mild relation to those of the participants in today philosophical debate. This paper will argue that the difficulties of understanding the constructive/ principle theories opposition is a consequence of its ambiguity. Einstein's article was, so to speak, at the same time the outline of (a) an ars iudicandi that supply criteria for justification of existing theories; (b) an ars inveniendi, a set of techniques for the discovery of new theories starting from the known ones. The reason of this ambiguity must be probably sought in the motivations with which the 1919 London Times was written. As this paper will try to show, the article should be read not as much as an abstract philosophical reflection, but as a personal testimony of a practicing physicist. As Einstein once wrote jokingly to his friend Paul Ehrenfest, he was, with few others, a principle-pincher 
(Prinzipienfuchser) ${ }_{1}^{1}$ ready to squeeze as much as possible from few fundamental principles, rather than a profligate virtuoso, squandering his calculation mastery in trifling puzzle solving (Einstein to Ehrenfest, Sep. 18, 1925; CPAE Vol. 15, Doc. 71; see Seth, 2010 ch. 6).

It was a paper by Francisco Flores, 1999 that, more than two decades ago, attracted new attention toward Einstein's distinction between constructive/principle theories by recasting in it into the opposition between two forms of explanations, bottom-up and top-down explanations (see also Dieks, 2009; Dorato, 2011; Felline, 2011). Soon thereafter, Jeffrey Bub (2000) suggested that quantum mechanics, just like special relativity, could be regarded as a 'theory of principles.' If the latter was a modification of Newton-Galilei kinematics that satisfied the light and relativity postulates, matrix mechanics was a modification of classical kinematics that complied with the quantum postulate and the correspondence principle. Quantum mechanics could be then seen as a set of information-theoretic constraints on possible dynamical theories (Clifton, Bub, and Halvorson, 2003). This proposal remained marginal in the literature about the foundation of quantum mechanics. However, at about the same time, Einstein's distinction between principle and constructive theories became the center of a vaster and still living philosophical debate on the foundation of spacetime theories. When Einstein compared the relativity principle to the second principle of thermodynamics, it was argued, he meant to complain about its lack of explanatory power of special relativity. Einstein ultimately aimed to find a deeper-level theory that 'explains' the relativistic kinematics, just like Ludwig Boltzmann had relied on the kinetic theory to 'explain' the increase of entropy. Thus, Harvey Brown and Oliver Pooley (2006) famously suggested that special relativity should ultimately take the form of a constructive theory about the material structure of rods and clocks, not differently from Hendrik A. Lorentz's ether theory. Michel Janssen and Yuri Balashov (2003) reacted by claiming that special relativity had actually already found its 'Boltzmann'; Hermann Minkowski had already transformed it into a constitutive theory, a theory about the geometrical structure of spacetime; it is the latter that explains why rods and clocks behave like they do (Stevens, 2014). The claim that in Einstein's view 'principle theories' lack explanatory power has been challenged by Marc Lange (2014).

Partly stimulated by this debate, historically oriented scholarship has attempted to clarify Einstein's principle/constructive theories opposition (Howard, 2005) by showing how it was deeply rooted in the $19^{\text {th }}$-century physics' emphasis on the role of general principles (Howard, 2007, see also Stachel, 2000), an epistemological position well-exemplified by the work of Hermann von Helmholtz (Bevilacqua, 1993). Indeed, the German-speaking physics community appears at first sight as being traversed by the fault line between a principle-based, phenomenological approach defended by Gustav Kirchhoff or Max Planck and a model-based approach of British ascendancy pursued, for example, by Boltzmann (Darrigol, 2018). Nevertheless, Planck agreed with Boltzmann in rejecting the anti-atomism of Ernst Mach and of the energetists, like Wilhelm Ostwald and Georg Helm (Deltete, 1999. Deltete, 2012). However, Planck sided with Pierre Duhem (Bordoni, 2017) against Boltzmann in indicating thermodynamics and not mechanics as model for scientific practice (Wolff, 2010). The differences between the two fractions were ultimately far from being clear-cut. Nevertheless, at the turn of the century, Lorentz (Frisch, 20052011 see also) and Henri Poincaré (Darrigol, 1995b), the other major protagonists of the relativity revolution, could present the opposition between the 'physics of principles' and the 'physics of models' as commonplace. In a similar vein, in the early $20^{\text {th }}$-century, Arnold Sommerfeld opposed a 'physics of problems,' a style of doing physics based on concrete puzzle solving, to the 'practice of principles' defended by Planck (Seth, 2010). As early as 1909, Philip Frank (1909), relying on a distinction introduced by Abel Rey (1908), had already explicitly classified relativity as a 'conceptual theory' like thermodynamics, rather than a 'mechanical theory' like the kinetic theory of gases. In 1910, Philip Lenard (1911) made a similar remark but using the less common opposition between two types of models, phenomenological models (relations between measurable quantities) and proper models (aether, electrons, etc.).

Thus, following the philosophical debate, one might be lead to the conclusion that Einstein's 1919 distinction between principle and constructive theories represents Einstein's fundamental insight into the nature of spacetime. On the contrary, by reading the historical literature, one might get the opposite impression that Einstein's distinction was a rather unoriginal variation on a threadbare $19^{\text {th }}$-century theme, with which, by 1919 , every working physicist was familiar (see, e.g., Sommerfeld, 1915). Both stances grasp indeed part of the truth but also miss what, in my view, is the fundamental point. As a matter of fact, in spite of the recent spike of interest, Einstein's principle/constructive theory distinction is often referred to but little investigated. Even the most wellinformed accounts (Ryckman, 2017. 9ff.) ultimately linger mainly on Einstein's somewhat colorless formulation of the principle/constructive theory opposition in Einstein's 1919 Times paper. However, Einstein had used the comparison between relativity theory and thermodynamics in a more incisive way much earlier amid the polemics surrounding special relativity, and returned to it on other occasions till the end of his life. The textual evidence is, however, rather scanty and made by isolated and elliptic remarks that have often been used and abused to underpin this or that philosophical pronouncement. This paper aims to fill what I think is a gap in Einstein

\footnotetext{
${ }^{1}$ A Pfennigfuchser is a 'penny pincher'.
} 
scholarship and offer a more systematic overview of this documentary material.

As a result, the paper hopes to show that the constructive/principle opposition is, neither the pre-figuration of the modern antithesis between dynamical and geometrical explanations in spacetime theories, nor yet another instance of the reoccurring distinction between phenomenological and model-based theories. To properly understand Einstein's 'theory of theories' (Flores, 1999), one has to disentangle the two threads of its fabric. Einstein introduced not only a distinction between two types of theories, but also between two strategies for finding theories (Balashov and Janssen, 2003). To prove this point with some 'philological' accuracy, however, it is necessary to collect and interpret a somewhat confusing maze of historical documents. To prove this point with some 'philological' accuracy, however, it is necessary to collect and interpret a somewhat confusing maze of historical documents. As an orientation, it is useful to organize the available textual evidence in three successive phases. In the first phase (1), during the Swiss years (1905-1914), Einstein mostly used the relativity theory/thermodynamics analogy as a negative defensive argument to address the concerns of his opponents. Only in the second phase (2), during his Berlin years (1914-1919), Einstein started to transform the comparison into a positive heuristics guiding the discovery processes. In the third phase (3), in his late Princeton years (1933-1955), Einstein attempted to reinterpret the historical path to special relativity as an application of such heuristics. The present paper will analyze at length phase (1) (part 1 and part 2 ) and show how it leads to phase (2) (part 3 ), and thus, ultimately, to the 1919 article for the London Times. Phase (3) (part 4) offers Einstein's own retrospective overview on matter from his later years, and it allows to extract a more clear-cut philosophical lessons from the analysis of the historical material.

More in details, the paper will show how Einstein started to compare relativity theory to 'thermodynamics before Boltzmann,' somewhat in passing, on two occasions, between the Spring of 1907 (section 1.3) and the beginning of 1908 (section 1.4), to answer a rather specific objection. Einstein had pretended to derive the dependence of the 'electron"s mass on velocity by adapting Newton's dynamics of charged point particles to the relativity principle (section 1.2). If Planck and Minkowski embraced and defended such derivation, Einstein had to justify it against who, like Ehrenfest, Sommerfeld and Abraham who deemed it as unsatisfactory. Einstein tried to argue that, if compared to previous electron theories, special relativity was like thermodynamics, a theory based on empirical principles that forgoes any assumption about the structure of matter or radiation but puts constraints on them. The argument, however, convinced only those who were already convinced. Even relativists like Max Born continued to deem necessary to search for a relativistic electromagnetic electron model (section 2.1) that would explain mass-velocity dependence (section 2.2). Only at the beginning of 1911, Max Laue (soon to be ennobled in von Laue) was able to prove that no model of the electron was necessary since any closed system in equilibrium behaves like a point particle in relativity theory (section 2.3 ). Einstein's view that the relativity principle was nothing but a constraint on the laws of nature became mainstream. In the Berlin period, while moving to gravitational research, Einstein transformed his defense strategy into a 'logic' of physical discovery (section 3.1). The 1919 London Times article, written in occasion of the experimental confirmation of the theory, lend Einstein the possibility to organize these reflections into the distinction between constructive and principle theories (section 3.2). During the Princeton years (section 4.1), Einstein was requested by several interlocutors to reconstruct the path that lead him to his 1905. He suggested that he had consciously decided to develop relativity following the example of thermodynamics because of his skepticism towards the exact validity of Maxwell equations. The Lorentz transformations are not the 'byproduct' of Maxwell equations or of any particular dynamical law; they are 'constraints' that all laws of nature have to satisfy.

Since the documentary evidence is relatively little, this paper is mostly devoted to inserting this material into the proper historical setting. In the history of science, an argument is often a counterargument (Beller, 1999). To understand the meaning of Einstein's relativity theory/thermodynamics analogy, one needs to grasp in response to which objections the analogy was introduced. In particular, it is necessary to understand how relativity theory was initially received (Walter, 2018) against the background of fin de siècle electron theories (Illy, 1981 Janssen, 2006: Miller, 1981) and their experimental verification (Cushing, 1981 Hon, 1995 Potters, 2019). However, the reader should be warned that the goal of the paper is not to reconstruct the technical aspects of these theories, which have been treated elsewhere in great details (see, e.g., Miller, 1986). On the contrary, this paper aims to highlight the epistemological discussions that gravitated around those technical details. Since, especially in early relativistic debate, the epistemological considerations of physicists were rarely exposed systematically, they have to be disentangled from the thicket of their technical concerns. It was after this debate that Einstein transformed the thermodynamics/relativity theory analogy from a classification of theories that are already available into a taxonomy of theories still to be discovered. As it has been rightly pointed out, the distinction between constructive and principle theories is, at the same time, a distinction between two types of theories (Flores, 1999) and two types of strategies of finding new theories (Balashov and Janssen, 2003).

Although the terminology is somewhat fluid, the average German-speaking physicist of the 'Einstein's generation' (Staley, 2008) conceived a physical theory (particle dynamics, hydrodynamics, electrodynamics, 
etc.) as a set of dynamical laws, which usually take the form of total (Newton's laws of motion) or partial differential equations (Maxwell equations, Euler equations, etc.). Given certain specific conditions that have to be introduced by hand, such equations might have solutions which approximate the behavior of observable (pendulum, inviscid fluid, etc.) or theoretical (the ether, the electron, the solar system, etc.) target physical systems. Such solutions might be called models (Bilder or Modelle) of the physical system (heliocentric model of the solar system, mechanical models of a gas, mechanical or electromagnetic models of a resonator, etc.). One might need more than one theory to construct a model (electromechanical model of the atom). Theories are 'successful,' if they allow constructing Bilder that accounts for a specific domain of observable phenomena (thermal, electromagnetic, gravitational phenomena, etc.). If this is not the case, one might try to construct more adequate models within the same theory (one thinks, for example, of the long list of mechanical models of the ether). Physics, some argued, must ultimately seek to construct a Weltbild, a 'model of the world' encompassing the totality of the phenomena, possibly based on a single theory (mechanical, electromagnetic worldview, etc.).

If available theories systematically fail to deliver models of observable phenomena (black-body radiation, ether-drift experiments, etc.), physicists might be forced to search for new ones that, by preserving the successes of the old theories, incorporate the new available evidence. Without making any claim of originality, Einstein pointed out that, in such circumstances, physicists use to apply two different stratagems. They might attempt to modify the theory's dynamical laws by trial and error in the hope that some new theory allows for the construction of more adequate models of the phenomena ('constructive strategy'). However, in proceeding in this way, one is often spoiled for choice among a large number of possible alternatives. In these circumstances, Einstein considered advisable to proceed indirectly and first restrict the number of possibilities. One singles out well-established empirical facts (no perpetuum mobile, no ether-drift, etc.) that can be expressed mathematically in the form of general principles (energy principle, relativity principle, etc.). These principles serve as constraints that all laws of nature have to satisfy if those facts have to hold. If some well-established dynamical law fails the compatibility-test, one can find a new law by modifying it so that it conforms to the principle. One can then establish whether these newly found dynamical laws allow for solutions that can serve as models for the observable phenomena.

In spite of his reputation of being a positivist, there is good evidence that Einstein always considered the goal of physics as the construction models. Physicists, as Einstein puts it on a few occasions, are like someone who tries to understand how a watch works but cannot open its unbreakable case (Einstein, 1925b Einstein and Infeld, 1938 33). They are not content of predicting the behavior of the visible parts of the watch, they want to understand why they behave as they do. Since the case of the watch is sealed, to understand how the watch works they need to formulate a 'theory' that allows constructing a hypothetical 'model' of its internal mechanism. The problem is how such a theory can be discovered. One might proceed in a constructive way, by trying out different models in the hope to end up with a good one; however, one might also proceed indirectly, by first searching for a principle that restricts the number of allowable models from the outset. The constructive/principle opposition was Einstein's attempt at a sort of 'logic of discovery' (Schickore, 2018), an outline of two strategies for searching for constructive theories. Most, if not, all of Einstein's scientific successes were obtained by following the principle strategy (in particular special and general relativity theory). Most, if not all, of his failures, happened when he was forced to fall back to the constructive strategy (from his early nonlinear non-Maxwellian electrodynamics to his late unified field theories). The constructive/principle opposition might be in itself disappointingly unoriginal. Nevertheless, it allows us to share a glimpse behind the wizard's curtain, to unveil his most successful trick: instead of directly searching for new theories, first search for the "formal conditions which constraint [einschränken] the number of possible theories" (Einstein to Seelig, Jan. 1, 1952; EA 8-311).

\section{Part 1}

Relativity theory was initially considered as a contribution to a debate about which model of the electron better explains the variability of the mass of the electron observed in experiments on cathode and $\beta$-rays. Einstein started to use the relativity theory/thermodynamics analogy to address the concerns about his derivation of the velocity-dependence of the mass of the 'electron.' Ehrenfest, Sommerfeld, and Abraham argued that Einstein's derivation was at least incomplete without making some hypotheses about the shape, charge distribution, and the nature of the mass of the electron. On the contrary, Planck and Minkowski endorsed Einstein's derivation as the result of the relativistic modification of Newtonian dynamics for charged point particles. Einstein's relativity/thermodynamics analogy did not seem to have won over the skeptics.

\subsection{Einstein and The Dynamics of the Slowly Accelerating 'Electron'}

In the spring of 1905 , in a famous letter, the 26 -year-old Einstein promised his friend Conrad Habicht four papers (Einstein to Habicht, May 18, 1905; CPAE Vol. 5, Doc. 72). As is well-known, Einstein defined the first of the series dealing radiation and energy property of light as "very revolutionary" (Einstein to Habicht, May 18, 1905; CPAE Vol. 5, Doc. 72). At that time, there was little doubt that matter should be described by Newtonian mechanics of discrete point particles, whereas vacuum Maxwell electrodynamics was based on the idea of the continuous field. The latter, Einstein pointed out, was so successful is accounting for optical phenomena, that it "will probably 
[whohl] never be replaced by another theory" (Einstein, 1905d, 132). Nevertheless, it could not be excluded that the even vacuum Maxwell equations were only macroscopic equations and that the microstructure of radiation was actually granular. Several phenomena, like black-body radiation, photo-luminescence seemed more easily explained by this assumption. Thus, Einstein wanted to explore this possibility, although he could not produce "any model [Bildes] for the production and propagation of radiation" (Einstein, $1905 \mathrm{~d}$ 137). The result was obtained by using as a heuristic guide the so-called 'Boltzmann's principle' relating the entropy of a system and the probability of its state. Einstein's strategy in this paper was analogous to the one he used in the second and third paper he mentioned to Habicht (Einstein to Habicht, May 18, 1905; CPAE Vol. 5, Doc. 72)-respectively the one based on his dissertation (Einstein, 1905a) on new method for measuring the size of atoms (Einstein, 1906c) and Brownian motion (Einstein, 1905c): calculate the fluctuations of observable macroscopic parameters (temperature, energy, density, etc.) in order to gain information about the micro-structure of matter or radiation (Norton, 2006).

The fourth paper that Einstein announced in the latter was "only a rough draft", unrelated to Einstein's statistical work. It was "an electrodynamics of moving bodies which employs a modification of the theory of space and time" (Einstein to Habicht, May 18, 1905; CPAE, Vol. 5, Doc. 72). In particular, Einstein imagined that Habicht would have appreciated the "purely kinematic part" of paper (Einstein to Habicht, May 25, 1905; CPAE Vol. 5, Doc. 72). The paper, which Einstein submitted in June 1905, opened, as we all know, with the celebrated magnet and a conductor thought experiment. The experiment was meant to show that Maxwell-Lorentz electrodynamics, "when applied to moving bodies" leads "to asymmetries which do not appear to be inherent in the phenomena" (Einstein, 1905d 891). A similar case of a non-observable asymmetry in Maxwell-Lorentz theory, Einstein pointed out, was the systematic failure of ether drift experiments of the first order in singling out a rigid reference body, among those in relative rectilinear uniform motion. This seems to suggest that "phenomena of electrodynamics as well as of mechanics possess no properties corresponding to the idea of absolute rest" (Einstein, 1905d 891). In all coordinate systems in which the equations of mechanics are valid, so are the laws of electrodynamics and optics. Einstein raised "this conjecture [...] to the status of a postulate" (Einstein, 1905d 891). He then added "another postulate apparently incompatible with the first", namely the source-independence of light speed (Einstein, 1905d. 892) which is implied by a wave theory of light (Norton, 2004).

Einstein famously removed such incompatibility by introducing a new kinematics of the rigid body in parallel uniform translation. There were good reasons to proceed in this way. Electrodynamics, and in general "each and any theory", concerns "the relations between rigid bodies (coordinate systems), clocks, and electromagnetic processes" (Einstein, 1905e 892). The position of a particle, the values of the components of a field, such as the electric or magnetic field, are usually defined with respect to three spatial and one time coordinates. In turn, a Cartesian coordinate scaffold is nothing but a rigid body endowed with clocks synchronized using electromagnetic signals. In the first part of the paper, by showing how this synchronization procedure would give different results in frames in uniform parallel translation, Einstein was able to provide a new kinematic definition of what counts as a rigid body. In geometry, a body is defined as rigid, if the distance between its points remains unchanged. However, in kinematics, measurements of length are supposed to be between simultaneous points. In the old kinematics, two points that are simultaneous in a body at rest were expected to be simultaneous on a body in motion. Einstein denounced this assumption as unanalyzed prejudice. By questioning the basic assumptions of Newtonian kinematics-the absolute character of temporal simultaneity and of spatial configurations-, Einstein could remove the apparent incompatibility of the two postulates. He introduced a new set of transformation equations (which turned out to be identical to those found by Lorentz) connecting the coordinates $x, y, z, t$ and $x^{\prime}, y^{\prime}, z^{\prime}, t^{\prime}$ of an event with respect to two rigid coordinate systems $K$ and $K^{\prime}$ in relative uniform translational motion. These equations satisfy the condition that the velocity of light has the same value $c$ with respect to the two systems (Einstein, 1905e 897ff.):

$$
t^{\prime}=\gamma\left(t-\frac{v}{c^{2}} x\right) \quad x^{\prime}=\gamma(x-v t) \quad y^{\prime}=y \quad z^{\prime}=z
$$

where $\gamma=1 / \sqrt{1-\beta^{2}}$ and $\beta=v / c$. Once one interprets the variables $x, y, z, t$ as the results of rods-and-clocks measurements, these transformation equations have direct physical consequences. A rigid spherical body of radius $a$, if measured in the rest system $K$, would appear like an ellipsoid with axis $a \sqrt{1-\beta^{2}}, a, a$ if measured from the frame $K^{\prime}$ in uniform parallel translation along the $x$-axis (Einstein, 1905e 903). A clock moving uniformly with velocity $v$ with respect to a reference system $K$ runs, as observed from this system $1 / \sqrt{1-\beta^{2}}$ slower than an identical clock that is at rest with respect to this system. These implications are, in principle, empirically testable. One needs only to find systems in nature that, when taken as the rigid rods and clocks of the theory, bear out its predictions.

After having "derived the necessary propositions of the kinematics that corresponds to our two principles", Einstein proceeded "to show their application" to the available dynamical laws (Einstein, 1905e 907). The first set of laws considered by Einstein were Maxwell-Hertz equations for empty space. Einstein showed that if these 
equations are valid with respect to $K$ they are also valid in the system $K^{\prime}$, if the transformations law of $\mathbf{E}$ and $\mathbf{B}$ with respect to the Lorentz transformation are defined as follows:

$$
\begin{array}{rr}
E_{x}^{\prime}=E_{x} & B_{x}^{\prime}=B_{x} \\
E_{y}^{\prime}=\gamma\left(E_{y}-\frac{v}{c} B_{z}\right) & B_{y}^{\prime}=\gamma\left(B_{y}+\frac{v}{c} E_{z}\right) . \\
E_{z}^{\prime}=\gamma\left(E_{z}+\frac{v}{c} B_{y}\right) & B_{z}^{\prime}=\gamma\left(B_{z}-\frac{v}{c} E_{y}\right)
\end{array}
$$

A similar reasoning can be applied to Maxwell equations with sources, if the transformations law for charges $\rho$ and currents $\rho \mathbf{u}$ are appropriately defined. These equations, together with the assumption that the charge is "unchangeably attached to small rigid bodies (ions, electrons), form the basis of Lorentz's electrodynamics and optics of moving bodies" (Einstein, 1905d 916; my emphasis). Einstein could then demonstrate, that "with our kinematic principles take as a basis, the electrodynamic foundation of Lorentz's theory of the electrodynamics of moving bodies agrees with the principle of relativity". Only the interpretation of the dynamical variables appearing in these equations has changed.

After having derived further results in optics and electrodynamics, in the final §10 of the paper, Einstein applied the new kinematics to another dynamical law, Newton's equations of motion for an electron moving under the influence of an electromagnetic field. Einstein considers a coordinate system $K$ 'at rest' and a system $K^{\prime}$ moving with constant velocity $v$ in the positive $x$-direction. Let an electron be at rest at the origin of a system $K^{\prime}$. In $K^{\prime}$ the electromagnetic force on the electron will be purely electric. Thus, Newton's second law takes the form:

$$
\begin{aligned}
& m \frac{d^{2} x^{\prime}}{d t^{\prime 2}}=q E_{x}^{\prime} \\
& m \frac{d^{2} y^{\prime}}{d t^{\prime 2}}=q E_{y}^{\prime} \\
& m \frac{d^{2} z^{\prime}}{d t^{\prime 2}}=q E_{z}^{\prime},
\end{aligned}
$$

where $x^{\prime}$, the coordinates of the particle in the reference system $K^{\prime}$, as functions of time $t^{\prime}$, and $E_{x}^{\prime}$ the component of electromagnetic field with respect to $K^{\prime}$, relative to which the electron is at momentary rest. Using eq. [1] and eq. [2]. Einstein derived the equations in the frame $K$ with respect to which the electron moves at speed $\mathbf{v}$. The final result of this computation was:

$$
\begin{array}{r}
\frac{d^{2} x}{d t^{2}}=\frac{e}{\mu} \frac{1}{\gamma^{3}} E_{x} \\
\frac{d^{2} y}{d t^{2}}=\frac{e}{\mu} \frac{1}{\gamma}\left(E_{y}-\frac{v}{c} B_{z}\right) \\
\frac{d^{2} z}{d t^{2}}=\frac{e}{\mu} \frac{1}{\gamma}\left(E_{z}+\frac{v}{c} B_{y}\right) .
\end{array}
$$

In this way, Einstein obtained the expression for the force on an electron moving in an electromagnetic field as a consequence of the principle of relativity ${ }^{2}$ Differently from classical particle dynamics, the ratio of force to acceleration is not the same for the component parallel to the velocity as for those perpendicular to it; moreover, this ratio depends upon the velocity and is not constant as in pre-relativistic dynamics. The coefficient of proportionality between force and acceleration is the mass $\mu$. Thus, "following the usual approach" (Einstein, 1905e 918), Einstein calculated the 'longitudinal' mass $\mu_{\|}$and the 'transverse' mass $\mu_{\|}$of a slowly accelerating electron under the influence of an electromagnetic field. To this purpose, Einstein wrote the equations eq. [4] in the equivalent form:

$$
\begin{array}{r}
\mu \gamma^{3} \frac{d^{2} x}{d t^{2}}=e E_{x}=e E_{x}^{\prime} \\
\mu \gamma^{2} \frac{d^{2} y}{d t^{2}}=e \gamma\left[E_{y}-\frac{v}{c} B_{z}\right]=e E_{y}^{\prime}, \\
\mu \gamma^{2} \frac{d^{2} z}{d t^{2}}=e \gamma\left[E_{z}+\frac{v}{c} B_{y}\right]=e E_{z}^{\prime}
\end{array}
$$

where $e E_{x}^{\prime}, e E_{y}^{\prime}, e E_{z}^{\prime}$ are the components of the ponderomotive force. To give physical significance to these equations, Einstein made the following assumptions: (1) he defined $e \mathrm{E}^{\prime}$, the force measured (e.g., by a spring

${ }^{2}$ This is, of course, nothing but Lorentz force law, which appears as a consequence of the relativity principle. 
balance at rest) in $K^{\prime}$, as 'the force exerted on the electron'; (2) he maintained the Newtonian definition of force, according to which the acceleration of a particle is proportional to the applied force and mass $m$ is the constant of proportionality; (3) he assumed that the acceleration must be measured in $K$ with respect to which the electron is moving Under these conditions, he obtained the following values of the coefficients of proportionality between mass and acceleration:

$$
\mu_{\perp}=\frac{\mu}{\left(\sqrt{1-\beta^{2}}\right)^{3}} \quad \mu_{\|}=\frac{\mu}{1-\beta^{2}} .
$$

In other words, the electron's mass $\mu$ rises with velocity inversely as $\left(\sqrt{1-\beta^{2}}\right)^{3}$ and the transverse mass as $1-\beta^{2}$. However Einstein was aware that with "a different definition of force and acceleration we should naturally obtain other values for the masses" (Einstein, 1905e 919). The force which acts on the electron was measured in the system $K^{\prime}$ in which the electron is at rest, while the acceleration in the rest system $K$. This interpretation is advantageous since only measured quantities occur. However, by changing the right-hand side of eq. [5] one could have also chose $e \mathbf{E}$ as a definition of force in terms of $K$. One would have obtained a different expression for the transverse mass. Whatever one decides, as Einstein remarked, "these results concerning mass are also valid for ponderable material points" in general, since "a ponderable material point can be made into an electron (in our sense) by adding to it an arbitrarily small electric charge” (Einstein, 1905e 919; my emphasis). As we shall see, the expression 'electron (in our sense)' entails what is maybe the most peculiar feature of Einstein's derivation.

Finally, Einstein calculated the kinetic energy $W$ of the electron. The latter corresponds to the work done by an electrostatic force to increase the velocity of the electron (Einstein, 1905e 919). Assuming that electron emits not radiation (that is in the case of slow acceleration), the work done on an electron by the electrostatic force $e F_{x}$ acting parallel to the $x$-direction in accelerating an electron during the infinitesimal time interval $d t$ is given by $F_{x} d x$. Einstein used the expression of the longitudinal force $F_{x} \gamma_{3} a_{x}$. Integrating, $d W=F_{x} d x=\mu \gamma^{3} v d v$ from rest to speed $\mathbf{v}$, Einstein found that the kinetic energy $W$ of an electron of mass $\mu$ is given by:

$$
W=\int e F_{x} d x=\int_{0}^{v} \mu \gamma^{3} v d v=\mu c^{2}\left[\frac{1}{\sqrt{1-\beta^{2}}}-1\right] .
$$

Using the longitudinal mass $\mu \gamma^{3}$, Einstein obtained the now familiar expression $\mu c^{2}(\gamma-1)$. The integration constant $\mu c^{2}$ assures that, for small velocities, the classical expression for the kinetic energy $\mu=\frac{1}{2} m v^{2}$ is recovered. On the contrary, the kinetic energy approaches infinity as the velocity $v$ approaches the velocity of light $c$. Also this result, Einstein emphasized, "must be valid for ponderable masses as well" charged or uncharged, microscopic or macroscopic (Einstein, 1905e, 919).

In this way, besides the 'direct' consequences of the new kinematics (length contractions, the relativistic composition of velocity, etc.), Einstein was able to obtain 'indirect' consequences (the variability of mass with velocity), by applying the new kinematics to some well-established laws of nature valid for low velocities. In the case of the dynamics of the slowly accelerating electron, the possibility of an experimental test of such relativistic effects was at hand. In particular, it was possible to measure the functional relationship between three quantities concerning fast moving electrons: the generating potential or or the kinetic energy of electrons, their deflectability by an electric field, and their deflectability by a magnetic field (Einstein, 1905e 920). "These three relations are a complete expression of the laws by which the electron must move according to the theory presented here" (Einstein, 1905e 921). At this point, a nearly unnoticeable semantic change occurs. The 'electron in our sense of the word' is replaced by the 'electron' in the 'in the usual sense of the word,' that is the negative charged particle moving at high velocity in cathode rays. The latter 'electrons,' because of their high velocity, indeed offer the only concrete possibility to test the predicted variability of mass. As we shall see, this semantic ambiguity of the word 'electron' will be the source of numerous confusions in the early reception of relativity theory (Einstein, 1905e 921).

At the end of September of 1905, a few months after publishing this famous papers Einstein (1905b), made the first step to go beyond the dynamics of particles towards the dynamics of the rigid body. "One more consequence of the electrodynamic paper has also occurred to me," he wrote to Habicht. "The argument is amusing and attractive, but I can't tell whether the Lord isn't laughing about it and playing a trick on me" (Einstein to Habicht, Jun. 28, 1905; CPAE Vol. 5, Doc. 28). Einstein's derivation offers a first example of the relativistic complications inherent in moving from the dynamics of point particles to that of an extended system. Einstein started from the assumption of the validity Maxwell-Hertz equations for empty space (which also imply the constancy of $c$ ) and the relativity principle. He considered the change in kinetic energy of a rigid body when it emits a pair of light pulses in opposite directions from the perspective of two inertial systems. Einstein made the crucial, but problematic assumption that the kinetic energy of rigid body "depends on the velocity exactly like the kinetic energy of the electron" (Einstein, 1905b; see Ohanian, 2009). Since process must satisfy the energy principle with respect to 
both the rest and the moving coordinate systems, Einstein concluded that, when a body releases the energy $L$ in the form of radiation, its mass decreases by $L / c^{2}$ (Einstein, 1905b 641). In this way, starting from Maxwell equations and the principle of energy conservation, Einstein obtained a further consequence of the application of the new kinematics: the inertia of a body depends upon its energy content, that is a change in energy results in a change in mass (Einstein, 1905b 641). Although $L / c^{2}$ is very small with respect to the mass, in principle, Einstein considered possible to test this implication of the relativity principle, since a noticeable decrease in mass should occur in bodies whose energy content is variable to a high degree, like radium.

\subsection{The Early Reception of Relativity Theory against the Background of Electron Theory}

\subsubsection{Kaufmann on Abraham's, Lorentz-Einstein's, and Bucherer's Electron Theories}

The young Einstein was initially disappointed by the "icy silence" with which his relativity paper was received (Pais, 1982 150). Planck, during the winter semester of 1905/1906, presented Einstein's electrodynamics of moving bodies at the physics colloquium in Berlin (Pais, 1982, 150). However, Einstein's (1905e) relativity paper was cited for the first time only in November of 1905 by the Göttingen experimentalist Walther Kaufmann (1905 954) in a short note presented at the plenary session of the Prussian Academy of Sciences. The note reported on Kaufmann's new experiments using Becquerel rays or $\beta$-rays emitted by radioactive substances. In earlier reports (Kaufmann, 1901 1902a b), Kaufmann had shown that the mass of the electrons moving in the $\beta$-rays of radium considerably grows as their velocity approaches that of light. The law of growth was in excellent agreement with the formulas calculated by Kaufmann's colleague in Göttingen Max Abraham (Abraham, 1902b c 1903) on the assumption that the electron was a rigid sphere, uniformly charged over its surface or its volume. Thus, this result "at first seems to justify the conclusion that the question after the constitution of the electron is definitely solved" (Kaufmann, 1905 950). However, this conclusion was premature.

As Kaufmann pointed out, a recent investigation by Lorentz, "led to the surprising result that an agreement with my previous experiments can be achieved using totally different assumptions on the electron" (Kaufmann, 1905 951). As is well known, Lorentz claimed that one could account for the failure of ether-drift experiments by constructing a deformable model of the electron. The model led to a different formula for the dependence of the electromagnetic mass on velocity, which was, however, compatible with Kaufmann's early observations. In this context, Kaufmann mentioned a "theory of electrodynamics recently published by Mr. A. Einstein [1905]". According to Kaufmann "Einstein's theory lead to consequences formally identical to those of Lorentz's theory" (Kaufmann, 1905 954; my emphasis). Although, none of this literature was cited in Einstein's (1905e) relativity paper, it was natural for Kaufmann to read its final section §10 as yet another contribution to an already wellestablished debate about the structure of the electron. Kaufmann gave a brief summary of electron theory in a longer version of his report published in the Annalen der Physik (Kaufmann, 1906). I will follow Kaufmann's presentation, adding some details that will be relevant to understand the reception of his paper.

Electron theory assumed that, in the space empty of matter and electricity, the Maxwell-Hertz equations hold, connecting the $t$ wo fields $\mathbf{E}$ and $\mathbf{B}$ to its sources, charges and currents. They postulate a system of reference $K$, the ether frame, in which plane electromagnetic waves in all directions spread with the same speed $c$. Motions related to this frame of reference are called absolute motions. The charge density $\rho$ is attached to discrete positive and negative particles called 'electrons.' In this sense, each electrical current of density $\rho \mathbf{v}$ is a convective current of moving electrons, which excites the same magnetic field B as the equivalent conduction current of Maxwell-Hertz's theory. The Newtonian force that electromagnetic field exerts on the electrons is additively composed of forces, acting in the electric field upon resting electrons, and in the magnetic field upon moving electrons $\mathbf{F}=\rho(\mathbf{E}+\mathbf{v} \times \mathbf{B})$ (the so-called Lorentz force law). Although the ether had no movable parts, it was still useful to think that the electron experiences a force because of the differential action of the so-called Maxwell stresses $\sigma_{i j}$ acting on it. By exerting forces on charged particles, electromagnetic fields change their kinetic energy. Energy conservation imposes that the energy supplied to the charged particle must have come from the electromagnetic field. The latter is assumed to possess an energy density $w$ (that is energy for unit volume) which is composed additively of an electric and a magnetic term $w=1 / 2\left(\mathbf{E}^{2}+\mathbf{B}^{2}\right)$. It was postulated that, when the density of energy increases in some places and decreases in other places, the flux of electromagnetic energy is given by $c$ times the vector product of $\mathbf{E}$ and $\mathbf{B}$, the so-called Poynting vector $\mathrm{S}=\mathrm{E} \times \mathbf{B}$.

According to Maxwell equations, a charged body produces through its charge an electric field $\mathbf{E}$, and through its motion, a magnetic field B. In turn, the latter, via Lenz law, increasingly 'tries to slow' the charged body's acceleration quicker it travels. Since 'inertial mass' is nothing but resistance to the change of velocity, a charged body should behave as if it had an 'apparent' mass in addition to the 'real' mechanical mass. A similar 'added mass' can also be found in hydrodynamics. When a body moves in a fluid, it drags some amount of fluid near it, so that it has a higher resistance to acceleration than the same sphere in a vacuum. For a macroscopic charged body, the additional 'electromagnetic' mass is negligible, but in the case of electrons, it was of the order of magnitude of the 
observed mass. The hypothesis could be made that the entire inertia of the electron was electromagnetic. In this way, mechanics could be reduced to electrodynamics (electromagnetic worldview), after the numerous attempts to reduce electrodynamics to mechanics had failed (mechanical worldview) (Wien, 1901, see McCormmach, 197ob). Early experimental tests made using cathode rays-beams of electrons in a vacuum glass tube moving from the negatively charged electrode (cathode) to positively charged electrode (anode)-did not confirm such variability of inertial mass. This negative result, Kaufmann continued, was, however, not surprising, since the velocity of electrons in cathode rays was too small to cause a noticeable mass-increase (Kaufmann, 1906 488-489).

Thus, new experiments were performed using $\beta$-rays emitted by the radium. The latter share the same properties of cathode rays (they are magnetically and electrically deflectable, and they carry an electric charge). However, their penetration capability is much higher, so that it can be assumed that electrons in $\beta$-rays move much faster (up to $90 \%$ the velocity of light). By examining how such rays are deflected by electric and magnetic fields, it was believed to be possible to establish to what extent this 'apparent' electromagnetic mass increases a function of the electron's speed respect to the 'real' mechanical mass. As Kaufmann (1906) recalled, this new experiments (Kaufmann, 1901) indeed confirmed not only a "very large mass-variability" in the electron, but also "that the portion of pure electromagnetic mass is very great if not overwhelming in comparison to a possible mechanic or material mass" (Kaufmann, 1906 488). However, "[ $\mathrm{t}]$ he attempt to calculate both shares did not lead to a substantial result" (Kaufmann, 1906 488-489).

Kaufmann had calculated the formula for the variation of the apparent mass of the electron from its electromagnetic field energy $W_{\mathrm{EM}}$. As long as an electron is at rest, only the electric energy is present $\frac{1}{2} \mathbf{E}^{2}$; when it moves, it generates a magnetic field, adding some magnetic energy to it $\frac{1}{2} \mathbf{B}^{2}$. As a consequence of this increase, the field energy of a moving electron is larger for a moving electron than for one that is at rest. If we are to put the electron in motion by an external force $\mathbf{F}$, we must not only produce the ordinary kinetic energy $1 / 2 m v^{2}$ but, in addition to this, the part of the electromagnetic energy that is due to the velocity. The kinetic energy of a particle is equal to the total work produced by the force that accelerates it. The effect of the self-field of the electron will, therefore, that a larger amount of work is required to accelerate a charged particle, than an ordinary material particle. Kaufmann relied on previous calculations for the total energy $W_{\mathrm{EM}}$ of a spherical body with a uniform surface distribution of charge in slow, uniform straight-line motion ${ }^{3}$ The change $d W_{\mathrm{EM}}$ of the kinetic energy of an electron is equal to the work $\mathrm{F} d x$ produced by an external force when the electron undergoes a displacement $d x$. Since $d x=v d t$, one can write $\mathbf{F}=(1 / v)(d W / d t)$. Defining mass as the ratio between force and acceleration Kaufmann could calculate the electromagnetic mass $\mu$ of an electron from its field energy :

$$
\mu=\frac{1}{v} \frac{d W_{\mathrm{EM}}}{d v} .
$$

From this formula, Kaufmann obtained the dependence on velocity of an electromagnetic mass of fast moving spherical electron 4 However, an agreement with the experimental observations could be obtained only by assuming that only a part of the electron mass was an 'apparent' variable electromagnetic $\mu$, and while another constant part was a mechanical (or 'real') mass $m$.

Abraham-a strong supporter of the electromagnetic worldview-challenged Kaufmann's pessimistic conclusion. The energy principle allows only to calculate "the 'longitudinal' mass" (Kaufmann, 1906, 489), the ratio of the force in the direction of motion to the acceleration in the same direction. However, a transverse force changing the electron's direction does not perform any work and does not change the energy of the electron; thus Kaufmann's calculation did not account for "the 'transverse' mass" of the electron (Kaufmann, 1906, 489). The forces in Kaufmann's experiment were exerted perpendicularly to the electrons' direction of motion; thus the transverse, and not the longitudinal, mass had to be taken into account. The latter could be derived not from the field-energy, but from the field momentum. Abraham suggested that, in order to save the principle of action and reaction in electrodynamics, the electromagnetic field should be attributed, in addition to the energy density

${ }^{3}$ According to Searle's (1897), the electromagnetic energy of a slowly moving spherical body of radius $a$ is

$$
\frac{e^{2}}{2 a}\left[\frac{1}{\beta} \ln \left(\frac{1+\beta}{1-\beta}\right)-1\right]
$$

Thus, the energy increases with velocity.

${ }^{4}$ Kaufmann's (1902b) expression for the apparent electromagnetic mass of a fast moving electron is derived from Searle's (1897) formula (footnote 3):

which reduces to

$$
\mu=\frac{1}{v} \frac{d W_{\mathrm{EM}}}{d v}=\frac{3}{4} \frac{\mu}{\beta^{2}}\left[-\frac{1}{\beta} \ln \left(\frac{1+\beta}{1-\beta}\right)+\frac{2}{1-\beta^{2}}\right],
$$

for the electron at rest.

$$
\frac{2}{3} \frac{e^{2}}{a c^{2}}
$$


$w_{\mathrm{EM}}$, a momentum density $\mathrm{g}_{\mathrm{EM}}$ (a momentum per unit volume) which is $1 / c^{2}$ times the Poynting vector $\mathrm{S}$, so that $\mathrm{S}=c^{2} \mathbf{g}_{\mathrm{EM}}$. The nine components of the Maxwell stresses are nothing the flow of the $x-, y-$ and $z$-components of the electromagnetic momentum density in the $x-, y-$ and $z$-direction. The force acting on the electrons enclosed in a portion of space produces an increase in momentum, which is balanced by a decrease of the field momentum. The total momentum of a moving electron $\mathrm{G}_{\mathrm{EM}}$ is obtained by integrating $\mathbf{g}_{\mathrm{EM}}$ from the surface of the electron to infinity $\mathrm{G}_{\mathrm{EM}}=\int \mathrm{g}_{\mathrm{EM}} d V$. In the case of an electrons in uniform velocity or quasi-stationary motion one can define force as product of mass and acceleration. One then obtains a different dependence of mass on velocity, if one divides the electromagnetic momentum for the velocity (longitudinal mass $\mu_{\|}$), or if one differentiate it with respect to the $v$ (transverse mass $\left.\mu_{\|}\right)$:

$$
\mu_{\|}=\frac{d \mathrm{G}_{\mathrm{EM}}}{d v} \quad[8 \mathrm{a}] \quad \mu_{\perp}=\frac{\mathrm{G}_{\mathrm{EM}}}{v}
$$

If one still insists on defining mass in the usual way as the coefficient of proportionality between mass and acceleration, the electromagnetic mass is not a scalar, but a 'tensor triple' (Abraham, 1905b, 194).

Abraham aimed to derive the dynamics of the electron, that is, its motion in a given external field, without resorting to the principles of mechanics, by assuming that the momentum of the electron was nothing but the momentum of its self-field. To this purpose, Abraham showed how the electron dynamics like the dynamics of particles, could be put into a Lagrangian form at least for electron moving uniformly or in quasi-stationary motion, for slow accelerations. If the motion of the electrons is given at the instants $t_{0}$ and $t_{1}$, the law of the motion during the interval can be found, can be derived from the principle of least action, by assuming that the time integral of the electromagnetic Lagrangian $L_{\mathrm{EM}}$ (the difference between kinetic and potential energies) of a moving electron is an extremum. $L_{\mathrm{EM}}$ is obtained by spatial integration of the difference between electric and magnetic energies densities over the whole volume external to the surface of the electron. From the Lagrangian, one can calculate (as in classical mechanics) the momentum $\mathrm{G}_{\mathrm{EM}}=d L / d \mathbf{v}$ (the derivative of the Lagrangian with respect to the velocity) and the energy $W_{\mathrm{EM}}=\mathrm{vG}_{\mathrm{EM}}-L$ of the electron. ${ }^{5}$ From the energy, one can calculate the longitudinal mass (eq. [7]) and from the momentum, both, the longitudinal (eq. [8a]) and the transverse mass (eq. [8b]). As Kaufmann explained, in order to calculate the Lagrangian $L_{\mathrm{EM}}$ of the electron in motion, Abraham had to make a "basic kinematic assumption" (Kaufmann, 1906 489), that is, he had to construct a specific model of a purely electromagnetic electron.

The electron must be extended, otherwise the electromagnetic field energy would become infinite. The field of the electron spread indefinitely outside of this volume, whereas within this volume, is either zero (surface charge distribution) or decreases according to a certain law (volume charge distribution) (Kaufmann, 1906, 489). Since equal charges repel each other, the charge was supposed to be attached to a material framework that counterbalances the enormous electrostatic repulsive pressure. However, if the volume of the electron is constant, no 'work of deformation' is done by such forces in changing its shape, and therefore they could be neglected. The rate of work of external forces Fv in accelerating the electron is equal to the rate of increase of the total electromagnetic energy. Thus, according to Abraham, only if the electron is rigid, a purely electromagnetic foundation of mechanics is possible, in which kinetic energy reduced to field energy. However, although the electron is not capable of any change of its shape, its behavior still depend s on which shape it has. If an electron is moving along the $x$-axis with velocity $v_{x}$, the motion of the corresponding electric field $\mathrm{E}$ gives rise to a magnetic field $B$, and their coexistence leads both to an energy flow $S$ and to electromagnetic momentum $\mathrm{G}_{\mathrm{EM}}$. These two vectors are parallel to the velocity $\mathbf{v}_{x}$ only if the distribution of charge is symmetrical around an axis parallel

${ }^{5}$ Abraham wrote the Lagrangian $(L=T-U)$ of the electromagnetic field as:

$$
L_{e}=\frac{1}{2} \int_{t_{0}}^{t_{1}}\left(\mathbf{B}^{2}-\mathbf{E}^{2}\right) d V,
$$

where the electron's kinetic energy is defined by $T=\frac{1}{2} \int \mathrm{B}^{2} d V$ and its potential energy $U=\frac{1}{2} \int \mathrm{E}^{2} d V$. Integration are taken to extend over the volume $V$ external to the surface of the electron. From the Lagrangian one can calculate the energy and momentum of the electron's electromagnetic field as in classical mechanics:

$$
\mathrm{G}_{\mathrm{EM}}=\frac{d L_{\mathrm{EM}}}{d v}=\int \mathbf{E} \times \mathbf{B} d V \quad W_{\mathrm{EM}}=v \frac{d L_{\mathrm{EM}}}{d v}-L_{\mathrm{EM}}=\frac{1}{2} \int\left(\mathbf{E}^{2}+\mathbf{B}^{2}\right) d V .
$$

An electron model is consistent with Lagrangian mechanics, only if the energy-derived and the momentum-derived longitudinal mass have the same value:

$$
\frac{1}{v} \frac{d W}{d v}=\frac{d G_{\mathrm{EM}}}{d v}=\frac{d^{2} L}{d v^{2}}
$$


to the $x$-axis. The electron's momentum has only one non-vanishing component $G_{x}$. If, on the contrary, the possibility of any non-symmetrical distribution of charge is left open, then the components $G_{y}$ and $G_{z}$ will, in general, not vanish. In order to guarantee the force-free motion of an electron in any direction, it was natural for Abraham to introduce the further assumption that the electron is spherical.

Thus, ultimately, Abraham constructed the electron as a "microscopic model [Bild]" (Kaufmann, 1906, 489) of a macroscopic charged spherical rigid body, like a pith-ball that has been brought into contact with the pole of an induction machine. The electron was simply supposed to obey the classical kinematics of the rigid body with its six degrees of freedom, and behave as a macroscopic charged rigid body would behave according to Maxwell's vacuum electrodynamics and Lorentz force law (Kaufmann, 1906 489). When $\beta$ is small, the energy of the moving sphere can be considered as equal to the mass at rest in the ether system $K$. It can be shown that the electromagnetic mass $\mu$ of a spherical electron is $4 / 3$ its electrostatic energy $W_{\mathrm{EM}}{ }^{6}$ In order to calculate the electromagnetic field of a rigid spherical electron in motion, Abraham resorted to a well-known artifice. He calculated the corresponding electrostatic field of an auxiliary electron at rest in the comoving system $K^{\prime}$, whose dimensions differ in a determinate manner from that of the real electron. Since the auxiliary electron is at rest, there are no magnetic field contributions, and we have only to calculate only the electric field. A rigid spherical electron of radius $a$ moving parallel to $x$-axis with respect to $K$, becomes, by substituting $x^{\prime}=x / \sqrt{1-\beta^{2}}$, a prolate ellipsoid at rest with axis $a / \sqrt{1-\beta^{2}}, a, a$ in the comoving system $K^{\prime}$. Abraham computed the electrostatic energy $W_{\mathrm{EM}}^{\prime}$ of such an auxiliary electron. The Lagrangian $L_{\mathrm{EM}}^{\prime}$ of the auxiliary electron at rest depends only $W_{\mathrm{EM}}^{\prime}$. The Lagrangian $L_{\mathrm{EM}}$ of the real spherical electron moving with respect to $K$ can be then determined as $L_{\mathrm{EM}}=L_{\mathrm{EM}}^{\prime} \sqrt{1-\beta^{2}}=W_{\mathrm{EM}}^{\prime} \sqrt{1-\beta^{2}}$. As we have mentioned, from the Lagrangian, one can obtain the momentum as $d L_{\mathrm{EM}} / d v$. From the momentum $\mathrm{G}_{\mathrm{EM}}$, using eq. [8a] Abraham calculated the formula for the transverse and longitudinal mass according to eq. [10] and obtained quite complicated logarithmic formulas.

After Abraham found the formula "for the transverse mass," Kaufmann continued, "the experiments have been repeated with improved setup, with the result that the measuring results are sufficiently represented by Abraham's formula" (Kaufmann, 1906 489). This was interpreted as an indication of a purely electromagnetic mass of the electron..$^{7}$ "The question after the constitution of the electron seemed to be decided" (Kaufmann, 1906

${ }^{6}$ The electric field $\mathrm{E}$ at radius $a$ is given by $\mathrm{E}=e / a^{2}$. Thus, integrating over the space outside of the electron (from $a$ to $\infty$ ), using the volume element $4 \pi r^{2} d r$, one obtains the electrostatic energy of a spherical electron:

$$
W_{\mathrm{EM}}=\int w_{\mathrm{EM}} d V=\int \frac{1}{2} \mathrm{E}^{2} d V=\frac{1}{2} \int_{\infty}^{a} \frac{e^{2}}{a^{4}} 4 \pi a^{2}=\frac{1}{2} \frac{e^{2}}{a c^{2}} .
$$

If the electron is moving through space with a uniform velocity slow compared that of light, associated with this moving electron there is a magnetic field $\mathbf{B}$, and thus a momentum density $\mathbf{g}_{\mathrm{EM}}=\mathrm{Sc}^{2}$. Using the law of Biot and Savart, $\mathbf{B}=\mathbf{E} \times \mathbf{v}$ and elementary vector identities, the momentum density of an electron moving moving along the $x$-axis is $g_{x}=v\left(E_{y}+E_{z}\right)$. For a spherically symmetric electron, $\int E_{z}^{2} d V=\int E_{y}^{2} d V=\int E_{z}^{2} d V=\frac{1}{3} \int \mathbf{E}^{2} d V$. Thus, $E_{y}+E_{z}=\frac{2}{3} \mathbf{E}^{2}$, where again $\mathbf{E}=e / a^{2}$. Integrating over the whole space outside of the electron:

$$
\mathrm{G}_{\mathrm{EM}}=\int \mathrm{g}_{\mathrm{EM}} d V=\int \frac{2}{3} \mathbf{E}^{2} d V=\mathbf{v} \int_{\infty}^{a} \frac{2}{3} \frac{e^{2}}{a^{4}} \cdot 4 \pi a^{2} d r=\frac{2}{3} \frac{e^{2}}{a c^{2}} \mathbf{v} .
$$

The momentum in the field is proportional to $\mathbf{v}$. We can, therefore, call this coefficient of the velocity the electromagnetic mass $\mu$ of the electron. By comparing footnote 6 and eq. [9] one obtains

$$
\mu=\frac{2}{3} \frac{e^{2}}{a c^{2}}=\frac{4}{3} \frac{1}{2} \frac{e^{2}}{a c^{2}}=\frac{4}{3} W_{\mathrm{EM}} .
$$

All theories of the electron agree on this value for the electromagnetic mass of a spherical electron at rest or moving slowly. It is obvious that this result in contrast with the relativistic formula.

${ }^{7}$ Abraham could already find the electrostatic energy of the prolate ellipsoid in the literature:

$$
W_{\mathrm{EM}}^{\prime}=\frac{1}{2} \int \mathrm{E}^{\prime 2} d V^{\prime}=\frac{1}{2} \frac{e^{2}}{a} \cdot \frac{\sqrt{1-\beta^{2}}}{2 \beta} \cdot \ln \left(\frac{1+\beta}{1-\beta}\right) .
$$

The Lagrangian of the moving electron is deduced from the electrostatic energy of the auxiliary electron at rest:

$$
L_{\mathrm{EM}}=\frac{1}{2} \int d \mathrm{E}^{\prime} d V^{\prime}=-W_{\mathrm{EM}}^{\prime} \sqrt{1-\beta^{2}}=-\frac{1}{2} \frac{e^{2}}{a} \frac{1-\beta^{2}}{2 \beta} \ln \left(\frac{1+\beta}{1-\beta}\right) .
$$

Energy and momentum of a moving spherical electron can be derived from the Lagrangian as shown in footnote 5

$$
\mathrm{G}_{\mathrm{EM}}=\frac{d L_{\mathrm{EM}}}{d v}=\frac{1}{2} \frac{e^{2}}{a c \beta}\left\{\left(\frac{1+\beta^{2}}{2 \beta}\right) \ln \left(\frac{1+\beta}{1-\beta}\right)-1\right\} \quad W_{\mathrm{EM}}=v \frac{d L_{\mathrm{EM}}}{d v}-L=\frac{1}{2} \frac{e^{2}}{a}\left\{\frac{1}{\beta} \ln \left(\frac{1+\beta}{1-\beta}\right)-1\right\} .
$$

Finally, from the energy and momentum, one can derived the longitudinal and and from the momentum transverse mass of the fast moving electron:

$$
\mu_{\|}=\frac{d \mathrm{G}_{\mathrm{EM}}}{d v}=\frac{1}{v} \frac{d W}{d v}=\frac{1}{2} \frac{e^{2}}{a c^{2}} \cdot \frac{1}{\beta^{2}}\left\{-\frac{1}{\beta} \ln \left(\frac{1+\beta}{1-\beta}\right)+\frac{2}{1-\beta^{2}}\right\} \quad \mu_{\perp}=\frac{\mathrm{G}_{\mathrm{EM}}}{v}=\frac{1}{2} \frac{e^{2}}{a c^{2}} \cdot \frac{1}{\beta^{2}}\left\{\left(\frac{1+\beta^{2}}{2 \beta}\right) \ln \left(\frac{1+\beta}{1-\beta}\right)-1\right\} .
$$

Note that the, the energy-derived and momentum derived longitudinal mass have the same numerical value.. 
490). The possibility of an electrodynamic foundation of mechanics appeared to be at hand. Abraham's (1903) dynamics of the electron was further developed by a group of Göttingen-based theoreticians who tried to move beyond the case of quasi-stationary translational motion. Since the electron has a finite extension, it should be capable also of rotation. Thus, Karl Schwarzschild (1903a 1903b 1903c), on the basis of his variational formulation of electrodynamics, extended Abraham's analysis to the case of quasi-stationary rotation. At the of 1903, Gusatv Herglotz (1903) calculated the field electron in arbitrary rotation. Arnold Sommerfeld (1904a 1904b 1905) was able to derive many of these results from a unified scheme, obtaining a what he called a 'supermecahnics' in which force-free oscillations and rotational motions were predicted that were excluded by 'ordinary mechanics' (Hertz, 1904 see Pyenson, 1985 117-129).

However, Kaufmann continued, in a 1904 paper, Lorentz (1903-1904), in the attempt "to remove the difficulties which still existed in the optics of moving bodies", suggested "somewhat modified fundamental assumptions about the electron and also about the molecular forces acting in-between the material body particles" (Kaufmann, 1906 490). Lorentz showed that Maxwell-Lorentz equations remain invariant under certain transformations (the Lorentz transformations). One could account for the negative results of ether-drift experiments (of second and higher orders), by assuming that, as prescribed by such transformations, the "dimensions of all physical bodies, including their individual molecules and electrons" contract by a factor of

$$
l=l_{0} \sqrt{1-\beta^{2}},
$$

while the transverse directions remain unchanged (Kaufmann, 1906, 490). Lorentz added the further assumption that "all molecular forces change in the same way with velocity as the electrostatic forces, and that the 'masses' of mechanics are changing the same way as the electromagnetic mass of the electron" (Kaufmann, 1906, 490-491). Like Abraham's electron, Lorentz's electron is a sphere with radius $a$ when at rest in ether system $K$; but it becomes an oblate ellipsoid $a \sqrt{1-\beta^{2}}, a$, $a$ when moving with uniform velocity with respect to $K$. Adopting the usual calculation trick, by substituting $x$ with $x^{\prime}=x \sqrt{1-\beta^{2}}$, Lorentz found that the auxiliary electron is again a sphere of radius $a$ at rest in the comoving system $K^{\prime}$. Lorentz calculated the electromagnetic momentum of such an auxiliary electron by integrating $\int \mathrm{g}_{\mathrm{EM}} d V^{\prime}$ over the volume outside of the sphere; by transforming back the result to $K$ he obtained the electromagnetic momentum $\mathrm{G}_{\mathrm{EM}}$ of the real contracted electron in motion through the ether. From it, it was straightforward to determine the velocity-dependence of the longitudinal and transverse mass in the usual way as in eq. [8a] and eq. [8b] ${ }^{8}$ Lorentz's formula was much simpler than the logarithmic relations found Abraham and it was compatible with Kaufmann early observations.

However, (Abraham, 1904a 1905b) immediately challenged Lorentz's deformable, electromagnetic electron. "Lorentz's deformation of the electron requires an expenditure of work" (Kaufmann, 1906 493). As a consequence of this 'work of deformation' of the constraining forces, the rate of work of the external forces in accelerating the (Fv) is not equal to the rate of increase of the total electromagnetic energy. Thus, the energy-derived longitudinal mass eq. [7] differs from the momentum-derived longitudinal mass eq. [8a] calculated by Lorentz. However, consistent equations should not deliver two different values for the same quantity ${ }^{9}$ In order, "to avoid a contradiction with

${ }^{8}$ Lorentz (1904) calculated the field $\mathrm{E}^{\prime}, \mathrm{B}^{\prime}$ for the system $K^{\prime}$ in which the auxiliary spherical electron is at rest. Then, the transformation formulas give the field $\mathbf{E}, \mathbf{B}$ in the system $K$, with respect to which the real contracted electron is moving. The $x$-component of the momentum of the moving electron along the $x$-axis with respect to $K$ has the form:

$$
G_{x}=\int\left(E_{y} B_{z}-E_{z} B_{y}\right) d V
$$

One then calculates the fields E' and B' and volume element $V^{\prime}$ with respect to $K^{\prime}$. In $K^{\prime}$ the auxiliary electron is at rest and there is no magnetic field $\mathrm{B}=0$. Since the auxiliary electron in $K^{\prime}$ is spherical, on integrating, the $y$ and $z$ components vanish, while the $x$ component becomes

By performing calculations analogous to those in footnote 6 one obtains:

$$
G_{x}=\frac{\beta}{c \sqrt{1-\beta^{2}}} \int\left(E_{y}^{\prime 2}+E_{z}^{\prime 2}\right) d V^{\prime} .
$$

$$
\mathrm{G}_{\mathrm{EM}}=\frac{2}{3} \frac{e^{2}}{a c^{2}} \frac{\mathbf{v}}{\sqrt{1-\beta^{2}}}=\frac{4}{3} \frac{1}{2} \frac{e^{2}}{a c^{2}} \frac{\mathbf{v}}{\sqrt{1-\beta^{2}}} .
$$

This is the formula electromagnetic momentum of a moving flattened electron. From it, Lorentz derived the values of the dependence of its longitudinal and transverse mass on velocity:

$$
\mu_{\|}=\frac{d \mathrm{G}_{\mathrm{EM}}}{d v}=\frac{4}{3} \frac{e^{2}}{2 a c^{2}}\left(\frac{1}{\sqrt{1-\beta^{2}}}\right)^{3} \quad \mu_{\perp}=\frac{\mathrm{G}_{\mathrm{EM}}}{v}=\frac{4}{3} \frac{e^{2}}{2 a c^{2}} \frac{1}{\sqrt{1-\beta^{2}}},
$$

for low velocity $\mu=\mu_{\perp}=\mu_{\|}$is $4 / 3$ is the electrostatic energy $W_{\mathrm{EM}}^{\prime}$ of a spherical electron.

${ }^{9}$ Abraham showed that the Lagrangian formalism is inconsistent with Lorentz electron model. The value of $\mathrm{G}_{\mathrm{EM}}$ derived by differentiating the Lagrangian with respect to the velocity, is not equal to the one calculated by Lorentz via direct spatial integration of the $\mathrm{g}_{\mathrm{EM}}$. According to Abraham (1905a) the Lagrangian of a moving deformable electron is: 
the energy law”, one must introduce an additional “inner potential energy' of the electron” of non-electromagnetic origin (Kaufmann, 1906 493-494). Instead of the work of an unknown inner energy of the electron, one could also introduced "unknown universal external pressure following Poincaré's proposal" (Kaufmann, 1906, 494) ${ }^{10}$ In this way, the total energy of a moving Lorentz electron would be not simply the electromagnetic energy, but the sum of the latter and the potential energy of the non-electromagnetic constraining forces. Under this assumption, the internal energy is again unaltered during a process of acceleration of a deformable electron. However, the "electromagnetic foundation of the mechanics of the electron by that also of mechanics in general" would be proved to be impossible (Kaufmann, 1906 494; my emphasis). Thus, if the new measurements should demonstrate the validity of Lorentz's theory, the ambitious project of an electromagnetic worldview should be abandoned (Kaufmann, 1906 494; my emphasis).

As Kaufmann (1906 494) pointed out, in order to obviate the need for additional non-electromagnetic energy and yet to maintain a theory of deformable electrons, a third electron model was suggested by Alfred Bucherer (1905) at the end of 1905 (see also Bucherer, 1904). Bucherer assumed that the spherical electron with radius $a$ at rest in the ether is, like that of Lorentz, deformed into an oblate ellipsoid. However, the perpendicular dimensions are increased, so that the electron's volume remains constant: $a{\sqrt{1-\beta^{2}}}^{\frac{2}{3}}, \quad a{\sqrt{1-\beta^{2}}}^{-\frac{1}{3}}, \quad a{\sqrt{1-\beta^{2}}}^{-\frac{1}{3}}$. As a consequence, Bucherer's auxiliary electron in the comoving system is again a sphere, but of dilated radius $a{\sqrt{1-\beta^{2}}}^{2 / 3}$. Since no work is done by the external forces accelerating the electron to change its volume, the total electromagnetic energy of Bucherer's electron remains constant. The value of the longitudinal mass calculated on the basis of eq. [7] coincides with the values found with eq. [8a] On the basis of this deformable, but incompressible electron model, Bucherer obtained yet another set of formulas for the velocity-dependency of the electron mass. However, the model could remove only some second-order effects of the absolute motion through the ether. "At this time, Bucherer is still concerned with the discussion of this question" (Kaufmann, 1906 494). Nevertheless, Kaufmann deemed appropriate to compare his experimental results with Bucherer's predictions as well.

Thus, only Lorentz's electron model could account for the experimental undetectability of ether-drift to any order in $\beta$, but, at expenses of an electromagnetic foundation of mechanics. However, Kaufmann regarded Lorentz's theory as quite unsatisfying from an epistemological point of view. It postulated that the electron is deformed, and its mass varies as a function of its absolute velocity with respect to the ether. However, such velocity has no physical meaning in Lorentz's theory. "It is now very remarkable," Kaufmann added, that, "starting from very different assumptions, Einstein recently arrived at results, which are in agreement with those of Lorentz for what the consequences accessible to observation are concerned", but avoiding the "mentioned difficulties of the epistemological kind" (Kaufmann, 1906 491-4922). Kaufmann, as it would soon become common in the debate, started to speak of the Lorentz-Einstein deformable electron and pit it against Abraham's rigid electron. However, Kaufmann offered a quite penetrating account of the difference between Lorentz's and Einstein's strategies of finding the same result.

Einstein, put the relativity postulate "at the top" (Kaufmann, 1906 492; my emphasis), and showed that the "the kinematics of the rigid body must necessarily be changed" (Kaufmann, 1906 492; my emphasis), if the postulate has to hold also for electrodynamics. On the contrary, "Lorentz only shows that his hypotheses" (Kaufmann, 1906 492; my emphasis) about the electron shape, the nature of the molecular forces, etc., happen to "lead to the desired

$$
L_{\mathrm{EM}}=-W_{\mathrm{EM}}^{\prime} \sqrt{1-\beta^{2}}=\frac{1}{2} \frac{e^{2}}{a c^{2}} \sqrt{1-\beta^{2}},
$$

where $W_{\mathrm{EM}}^{\prime}$ is the electromagnetic energy of the auxiliary spherical electron at rest in the comoving system $K^{\prime}$. Thus, the momentum calculated from the Lagrangian is:

$$
\frac{d L_{\mathrm{EM}}}{d v}=\mathrm{G}_{\mathrm{EM}}=\frac{1}{2} \frac{e^{2}}{a} \frac{1}{\sqrt{1-\beta^{2}}} \mathbf{v},
$$

which amounts to $3 / 4$ the value of momentum $\mathrm{G}_{\mathrm{EM}}$ found by Lorentz by direct integration. Thus the Lagrangian scheme is inconsistent:

$$
\frac{1}{v} \frac{d W}{d v}=\frac{d G_{\mathrm{EM}}}{d v}+\frac{1}{v}\left(\mathrm{G}_{\mathrm{EM}}-\frac{d L}{d v}\right) .
$$

where $\mathrm{G}_{\mathrm{EM}}$ is value of the electron momentum found by Lorentz..

${ }^{10}$ Kaufmann was probably one of the first German physicists to cite Poincarés four-page summary of his dynamics of the electron which appeared on the June 5, 1905 issue of the Comptes rendus of the Paris Academy of science. There, Poincaré had pointed out that Lorentz theory could be made consistent by assuming that the "deformable and compressible electron is subjected to a kind of constant external pressure whose work is proportional to the variations of the volume" (Poincaré, 1905 1506). This inward pressure would balance the outward pressure on the surface of the electron due to the electric repulsion between charges. The assumption of such a external pressure leads to a corresponding energy of non-electromagnetic origin. The sum of the $W_{\mathrm{EM}}+W_{\mathrm{COE}}$ remains constant, if the electron is accelerated by external forces. In his Palermo-memoir, Poincaré (1906) showed that, if one adds $1 / 3$ non-electromagnetic energy to the Lagrangian of Lorentz electron, the latter becomes $L_{\mathrm{EM}}+L_{\mathrm{COE}}=4 / 3 W_{\mathrm{EM}}^{\prime} \sqrt{1-\beta^{2}}$, instead of $L_{\mathrm{EM}}=W_{\mathrm{EM}}^{\prime} \sqrt{1-\beta^{2}}$. Thereby, the consistency of Lorentz model with Lagrangian formalism is reestablished (Bracco and Provost, 2009). Poincaré paper was published only in January 1906 (Poincaré, 1906) and Kaufmann could not know the details of Poincaré's argument. 
result", the undetectability of absolute motion, "without excluding that the same can also be achieved in another way" (Kaufmann, 1906 492; my emphasis). Thus, while Lorentz had constructed a suitable dynamical model of a non-rigid electron, Einstein had changed the general kinematic definition of a rigid body in general. The proper relativistic analogue to Lorentz's deformable electron model is a 'relativistically rigid electron.' For both, Lorentz and Einstein, a spherical electron at rest becomes an ellipsoid when it moves with respect to rest system $K$, and it is again a sphere with respect to a system $K^{\prime}$ comoving with the electron. However, by taking seriously Lorentz' $\mathrm{s}$ "local time" " (Kaufmann, 1906 492; my emphasis), as the real time, Einstein abolished the distinction between the 'real' deformed electron in motion with respect to $K$ and the 'auxiliary' spherical electron at rest in the comoving system $K^{\prime}$. The perspective of $K^{\prime}$ was declared as just as 'good' as that of $K$, and both electrons are 'real.'

After this brief introduction to the electron-theoretical debate, Kaufmann proceeded to compare the three available formulas for the dependence of the electron's transverse mass on the velocity with his experimental data. All three theories (Abraham, Lorentz-Einstein, Bucherer) started from the natural assumption that, when $\beta$ tends to 0 , the electron is spherical, and its mass $\mu$ is $4 / 3$ its electrostatic energy $W_{\mathrm{EM}}$. When $\beta$ becomes closer to 1 , the transverse mass increases $\mu_{\perp}=\mu \cdot \phi(\beta)$, where $\phi$ is a function of velocity. The form of $\phi$ form is different depending on the basic assumption concerning the constitution of the electron. In Kaufmann's notation:

$$
\begin{array}{rr}
\text { I. (Abraham) } \Phi(\beta)= & \frac{3}{4} \frac{1}{\beta^{2}}\left(\frac{1+\beta^{2}}{2 \beta} \cdot \lg \frac{1+\beta}{1-\beta}-1\right) \\
\text { II. (Lorentz) } \Phi(\beta)= & \left(1-\beta^{2}\right)^{-1 / 2} \\
\text { III. (Bucherer) } \Phi(\beta)= & \left(1-\beta^{2}\right)^{-1 / 3} .
\end{array}
$$

Kaufmann pointed out that "[ $\mathrm{t}]$ he theory of Einstein leads to the same formula as the one of Lorentz" (Kaufmann, 1906 530; my emphasis). It should be noticed that Einstein, differently from Lorentz, had found that the transverse mass of the moving electron was $1 / \sqrt{1-\beta^{2}}$ the rest mass and not $1 /{\sqrt{1-\beta^{2}}}^{2}$. However, Kaufmann seems to have been the first to point out that this discrepancy depended on Einstein's definition of force as mass times acceleration, and in particular on his choice to measure force with respect to to the system $K^{\prime}$ comoving with electron, and the acceleration with respect to rest system $K$. If the components of both the force and the acceleration are both measured in $K$, one obtains that the rest mass is $1 /{\sqrt{1-\beta^{2}}}^{2}$, the same value obtained by Lorentz. Thus, "the difference of his equation for the electron's mass from that of Lorentz" was not essential (Kaufmann, 1906, 530). In one case, one measures the force as $e \mathbf{E}^{\prime}$ in terms of $K^{\prime}$, in the second as $e \mathbf{E}$ in terms $K$. Both sets of equations express the same physical phenomena and predict the same paths of the electrons in the experimental apparatus.

However, Kaufmann's comparison between the predicted values and the observable results lead him to a seemingly unappealable conclusion. While his experimental results could not decide between Abraham's and Bucherer's models, they "resolutely speak against the correctness of Lorentz's and therefore also Einstein's theory" (Kaufmann, 1906 534; my emphasis). Thus, Kaufmann considered them "refuted" (Kaufmann, 1906, 534). As a consequence, "the attempt to base the entire physics, including electrodynamics and optics, on the principle of relative motion, must be denoted for the time being as unsuccessful" (Kaufmann, 1906, 534). One could maintain the relativity principle and obtain agreement with Kaufmann's results, only if

qtMaxwell's equations for the bodies are rest are modifiedbereits die Maxw ellschen Gleichungen fur ruhende Korper modifizieren miiBte toward a emission theory of light as it was suggested by Walther Ritz ${ }^{11}$ "a step to which hardly anyone will decide himself for the time being" (Kaufmann, 1906 534). Thus, Kaufmann preferred to concede that physical laws must be formulated respect "to a very special coordinate system, which we call the absolutely resting aether" (Kaufmann, 1906, 534). Although, the influence of the motions of bodies respect to the aether could not be determined by electrodynamic or optical experiments, "the impossibility of such a demonstration may not be concluded" (Kaufmann, 1906, 534).

\subsubsection{Early Reactions to Kaufmann's Data}

Many at that time considered the question settled by Kaufmann's results. Lorentz himself had confessed to Poincaré that he was at his wits' end (Lorentz to Poincaré, Mar. 8, 1906; SCHAL Vol. 1, Doc. 140). The younger generation of physicists was ready to move on. In a paper finished in March, Paul Ehrenfest-a Viennese physicist working on radiation theory (Ehrenfest, $1906 \mathrm{~b} \mathrm{a}$ ) in Göttingen-, without bothering to mention Einstein's theory, took for granted that Kaufmann's results had definitively refuted "Lorentz deformable electron" (Ehrenfest, 1906c 302). Only the choice between "Abraham's rigid electron and Bucherer-Langevin's deformable electron" remained open (Ehrenfest, 1906c 302). The stability of Abraham's electron was granted by the "purely kinematic conditions of rigidity" (Ehrenfest, 1906c 302). Abraham had shown that Lorentz's deformable electron was unstable since its

\footnotetext{
${ }^{11}$ Although, the name is not mentioned, I surmise that Kaufmann is referring to Ritz, who was a student of his in Göttingen. Ritz wanted to modify Maxwell equations in order to save the relativity principle. See below footnote 23
} 
internal potential energy increased by volume's reduction. Although the stability of Bucherer-Langevin's electron had not been properly investigated, Ehrenfest suspected that it "was probably unstable" as well (Ehrenfest, 1906c 302).

An electron of spherical shape in equilibrium at rest, with either surface or volume charge distribution, has a maximum of potential energy respect to any shape of the same volume. Thus, once Bucherer-Langevin the electron is deformed at constant volume, it cannot get back to its original shape. As as a consequence, such electron would dissolve under the effect of its electrostatic repulsive forces (Ehrenfest, 1906c 302-303). Ehrenfest's reflections are a good example of how, for Göttingen-based electron-theorists, the dynamics of the electron were inseparable from specific details about the shape, charge distribution, that is from a proper "dynamics of the deformation" that addressed the problem of the electron stability (Ehrenfest, 1906c 303; fn. see Klein, 1970 148-150 for more details). As Kaufmann had explained to Ehrenfest in private conversation, one could avoid this problem only by abandoning the assumptions that electron's energy and momentum were purely electromagnetic. One needed to postulate axiomatically that, the electron, whatever its constitution, satisfies the energy principle and the center-of-mass theorem (Ehrenfest, 1906c 303; fn. )

It was an older physicist like Planck who, at the session of March 23, 1906 at the Deutsche Physikalische Gesellschaft, invited the physics community not to be too hasty in drawing conclusions from "the new important measurements of W. Kaufmann” (Planck, 1906a 136). Planck insisted that the Lorentz-Einstein relativity principle presented significant advantages that made it worth further investigation (see Goenner, 2010 Goldberg, 1976). By keeping Newtonian equations of mechanics, Lorentz could show that it happens to be impossible to detect the earth's motion through the ether in any order of $v / c$, if one constructs a suitable semi-electromagnetic model of the electron, the deformable electron, with a suitable velocity dependence of mass. Planck preferred to follow Einstein's opposite strategy. The goal was "to determine the form of the fundamental equations of mechanics, which take the place of the usual Newtonian equations of motion of a free mass point [...] if the relativity principle should have general validity" (Planck, 1906a 137; my emphasis). According to this principle, those simple equations are only valid for a slowing moving mass points, whereas for point particles moving with arbitrary velocity $v^{2}=v_{x}^{2}+v_{x}^{2}+v_{x}^{2}$ they need to be modified.

Planck, like Einstein, considered a special case in which one knows the connection between the components of the moving force in both reference frames, i.e., the effect of an electromagnetic field in vacuum on a charged mass point $m$ and charge $e$. Planck derived Einstein's eq. [4] in the rest system $K$. However, at the end of his derivation, he made an essential additional move. By "a simple rotation of the coordinate axes" (Planck, 1906a 139), he managed to write the equations of motion in a way in which their symmetry becomes manifest:

$$
\begin{aligned}
& \frac{m a_{x}}{\sqrt{1-\beta^{2}}}=e\left(\mathbf{E}_{x}-\frac{e v_{x}}{c^{2}} v_{x} \mathbf{E}_{x}+v_{y} \mathbf{E}_{y}+v_{z} \mathbf{E}_{z}\right)+\frac{e}{c}\left(v_{y} \mathbf{B}_{z}-v_{z} \mathbf{B}_{y}\right) \\
& \frac{m a_{x}}{\sqrt{1-\beta^{2}}}=e\left(\mathbf{E}_{x}-\frac{e v_{x}}{c^{2}} v_{x} \mathbf{E}_{x}+v_{y} \mathbf{E}_{y}+v_{z} \mathbf{E}_{z}\right)+\frac{e}{c}\left(v_{y} \mathbf{B}_{z}-v_{z} \mathbf{B}_{y}\right) \\
& \frac{m a_{x}}{\sqrt{1-\beta^{2}}}=e\left(\mathbf{E}_{x}-\frac{e v_{x}}{c^{2}} v_{x} \mathbf{E}_{x}+v_{y} \mathbf{E}_{y}+v_{z} \mathbf{E}_{z}\right)+\frac{e}{c}\left(v_{y} \mathbf{B}_{z}-v_{z} \mathbf{B}_{y}\right) .
\end{aligned}
$$

Since these equations have a symmetric form relative to the three spatial variables, no quantities appear, which could be interpreted as longitudinal and transverse components. By scalar multiplication of eq. [10] by $v$, Planck obtained:

$$
e\left(v_{x} \mathbf{E}_{x}+v_{y} \mathbf{E}_{z}+v_{z} \mathbf{E}_{x}\right)=\frac{m\left(v_{x}^{2}+v_{y}^{2}+v_{z}^{2}\right)}{{\sqrt{1-\beta^{2}}}^{3 / 2}},
$$

substituting eq. [11] into eq. [10]

$$
\begin{aligned}
& F_{x}=e\left(E_{x}+v_{y} B_{z}-v_{z} B_{y}\right) \\
& F_{y}=e\left(E_{y}+v_{z} B_{x}-v_{x} B_{z}\right) \\
& F_{z}=e\left(E_{z}+v_{x} B_{y}-v_{y} B_{x}\right)
\end{aligned}
$$

where $F_{x}, F_{y}, F_{z}$ are defined as follows:

$$
\frac{d}{d t}\left\{\frac{m v_{x}}{\sqrt{1-\beta^{2}}}\right\}=F_{x}, \quad \frac{d}{d t}\left\{\frac{m v_{y}}{\sqrt{1-\beta^{2}}}\right\}=F_{y}, \quad \frac{d}{d t}\left\{\frac{m v_{z}}{\sqrt{1-\beta^{2}}}\right\}=F_{z},
$$

Planck defines force as the rate change of momentum rather than product of mass and acceleration. The relativity principle requires that the momentum assumes a more complicated form $G=\gamma m \mathbf{v}$ instead of the Newtonian form 
$m \mathbf{v}$ which is valid only for low velocity. The ambiguity of Einstein's formulation disappears. The right-hand side of eq. [12] is the only compatible with Planck's definition of force eq. [13] "These equations," Planck concluded his derivation, "contain the solution of the problem": "they form that generalization of Newton's equations of motion, which is required by the principle of relativity" (Planck, 1906a 137; my emphasis).

Finally, Planck showed, how this equations of motion of classical mechanics can be derived from the principle of least action, the Hamilton's principle. The real motion of a particle between two arbitrary times is distinguished by the condition that the integral:

$$
\int_{t_{0}}^{t_{1}}(\delta H+A) d t=0
$$

has an extremal value. In these equations, $\delta$ is arbitrary displacement of the independent coordinates (and velocities). $H$ correspond the what Planck, using a nomenclature introduced by Hermann von Helmholtz (1887), called the 'kinetic potential' of a system $H=T-U$ (that is its Lagrangian) and $A$ is the work corresponding to the variation $\delta$ which would be done on the system by the external forces. From the principle of least action one can derive one the following equations of motion:

$$
\frac{d}{d t}\left(\frac{\partial H}{\partial v_{x}}\right)=F_{x} \quad \frac{d}{d t}\left(\frac{\partial H}{\partial v_{y}}\right)=F_{y} \quad \frac{d}{d t}\left(\frac{\partial H}{\partial v_{z}}\right)=F_{z},
$$

which corresponds to Lagrange's equations in the pre-relativistic mechanics. The relativistic equations differ only in the definition of $H$. By assuming the invariance of $H / \sqrt{1-\beta^{2}}$ under Lorentz transformations, Planck showed how one can determine the dynamics of a single mass point in a vacuum with arbitrary velocity $v$, provided one knows something about the kinetic potential of the mass point for for low velocities. In the latter case, for a single particle acted on by external forces, the kinetic potential may be taken as the kinetic energy $1 / 2 m v^{2}$. In relativistic point dynamics, the kinetic potential takes the form $-m c^{2} \sqrt{1-\beta^{2}}$, an expression which, except for an additive constant, becomes identical with $1 / 2 m v^{2}$ in the limiting case. From the kinetic potential one can calculate the energy $W$ and momentum $\mathrm{G}$ of a free point particle moving with arbitrary velocity $\mathbf{v}$ :

$$
\begin{gathered}
\mathrm{G}=\frac{\partial H}{\partial q}=-\frac{v H}{c \sqrt{c^{2}-v^{2}}} \\
W=v_{x} \frac{\partial H}{\partial v_{x}}+v_{y} \frac{\partial H}{\partial v_{y}}+v_{z} \frac{\partial H}{\partial v_{z}}-H=\mathbf{v} \frac{\partial H}{\partial q}-H=-\frac{H}{\sqrt{1-\beta^{2}}}
\end{gathered}
$$

If one wants (see Planck, 1908b), one can reintroduce the distinction between the transverse mass $m_{\perp}=\mathrm{G} / v$ and the longitudinal mass $m_{\|}=d \mathrm{G} / d \mathbf{v}$ the longitudinal mass. For $v=0$, we have, $m=m_{\|}=m_{\perp}=H / c^{2}$. If one replaces in the above expressions the constant $H$ by the constant $m$, the momentum and energy of the free particle becomes:

$$
\mathrm{G}=\frac{m v}{\sqrt{1-\beta^{2}}} \quad W=\frac{m c^{2}}{\sqrt{1-\beta^{2}}}=m c^{2}+\frac{1}{2} m v^{2}+\cdots
$$

The familiar expression for the kinetic energy ordinary mechanics appears as expected as an approximate value. As we have seen $G / v$ is called the transverse mass $\mu_{\perp}$ and $d G / d v$ the longitudinal mass $\mu_{\|}$of the point

$$
m_{\perp}=\frac{m}{\sqrt{1-\beta^{2}}} \quad m_{\|}=\frac{m}{\left(\sqrt{1-\beta^{2}}\right)^{3 / 2}}
$$

In this way, Planck showed that, by modifying classical particle dynamics so that it complies with the relativity principle, he could recover the same values for the longitudinal and transversed mass obtained by Lorentz introducing a deformable electron model. However, the relativistic approach, according to Planck, had one clear advantage with respect to the one pursued by electron-theorists.

Planck's relativistic momentum was not the momentum of the electromagnetic field, but the momentum of a mass point in general, nor was Planck's kinetic potential $H$ was the electromagnetic Lagrangian. Thus, Planck's derivation was more general and encompassing. For this reason, Planck did not attach "decisive importance", to the fact that, according to the relativity principle, a moving electron would be subject to a "specific work of deformation" necessary to to flatten the electron (Planck, 1906a 137; my emphasis). One can postulate that the total energy of Lorentz's electron is unaltered during a process of acceleration, if one includes in the total energy, not only the electromagnetic energy, but also the potential energy of the constraining forces. In this way, the work of external forces in accelerating the electron can be "added to the balance [rechnen] of the kinetic energy 
of the electron"; the question of electron's constitution could be provisionally ignored (Planck, 1906a 137). It is true that, in this way, "[ $\mathrm{t}$ ]he question of an electrodynamic explanation of inertia remains open" (Planck, 1906a 137). However, the Einstein/Planck approach had the advantage that one does not have "to ascribe to the electron neither a spherical shape [Kugelgestalt] nor even any other shape in order to arrive at a certain dependence of inertia on speed" (Planck, 1906a 137; my emphasis).

As in the case of Einstein, Planck was fundamentally indifferent with regard to the question of the structure of the electron. 'Electrons' in cathode or $\beta$-rays were only relevant as tools to test the new relativistic equations for the motions of charged point particles in general under the influence of the electromagnetic field. In August, Einstein (1906b) himself suggested a different experimental setting for the determination of the ratio of the transverse and the longitudinal mass of of three models of the electrons (Abraham, Bucherer, and 'Lorentz and Einstein'). However-just promoted to technical expert, third class, at the Bern patent office-, he could not participate in the discussion on Kaufmann's experiments at the annual general meeting of the Gesellschaft Deutscher Naturforscher und Ärzte held in mid-September in Stuttgart. Using the formalism developed in the previous paper, in his Stuttgart address, Planck (1906c) compared the available electron theories by calculating the kinetic potential H of Abraham's Kugeltheorie (eq. [16a]) and Lorentz-Einstein's Relativtheorie (eq. [16b])--the two theories that were more developed:

$$
H=-\frac{3}{4} \mu c^{2} \frac{1}{\beta^{2}}\left[\frac{1+\beta^{2}}{2 \beta} \log \left(\frac{1+\beta}{1-\beta}\right)-1\right]
$$

$$
H=-\mu c^{2}\left(\sqrt{1-\beta^{2}}-1\right) .
$$

From $H$ one can then determine the conjugate momentum and energy of Abraham's and Lorentz's electron. As we have mentioned, three quantities concerning cathode and $\beta$-rays are accessible to precise observation: the potential difference producing the velocity of the rays (generating potential), the electrostatic deflection, and the magnetic deflection. From the formula for the momentum, Planck calculated the theoretical values the magnetic and the electrical deflectability for each electron model, and compared them with the values observed by Kaufmann (Planck, 1906b §3 and §4). Planck concluded that Abraham’s theory was indeed closer to Kaufmann’s experimental data. However, according to Planck this could not be interpreted as a final confirmation of Abraham Kugeltheorie and refutation of Lorentz' Relativtheorie. To draw this conclusion, the deviations of the theoretical numbers between Lorentz and Abraham's theory should be smaller than the deviations of any theoretical number from the observed ones. However, this is not the case (see Cushing, 2000 ch. 15).

A discussion was held after Planck's (1906c) lecture, to which among others, Kaufmann, Bucherer, Abraham, and Sommerfeld participated (Planck, 1906c). Kaufmann was glad that Planck had obtained the same theoretical values using a different method. For what the observed values were concerned, Kaufmann insisted that the deviations of the observed theoretical values were $10-12 \%$ for Lorentz theory, and only only $3-5 \%$ for Abraham. Planck pointed out that both results were nevertheless outside the observational error. However, Abraham, provoking the hilarity of the other attendees, commented that since "the deviations from the Lorentz theory are at least twice as big”, his theory represented Kaufmann's numbers "twice as well as the relativity theory", that is, the Lorentz-Einstein electron model (in Planck, 1906c 761). But, most of all, Lorentz-Einstein theory was not an "electromagnetic theory" (in Planck, 1906c 761), since the Lorentz-electron was not stable without adding a non-electromagnetic force. As as consequence, "cathode rays cannot be considered purely electromagnetic processes" (in Planck, 1906c 761).

"Abraham is right", Planck commented (Planck, 1906c 761). The choice between Abraham's Kugeltheorie and Lorentz-Einstein Relativtheorie was ultimately the choice between the electromagnetic worldview and the principle of relativity. Planck regarded the first approach as far too adventurous; by contrast, Sommerfeld did not share the "pessimistic viewpoint of Mr. Planck" (Planck, 1906c 761). The 38-year-old Sommerfeld famously replied to the ten years older Planck by predicting that physicists under forties would prefer the "electrodynamics postulate," while those over forty the, "mechanical-relativistic postulate" (Planck, 1906c 761). This tongue-in-cheek remark well represents the hopes that electrodynamic worldview continued to raise among most physicists working on electron-theory; relativity theory's silence towards the nature of the mass of electron appeared, by contrast, as a concession to the ponderable mass, and thus to the old-fashioned mechanical worldview. The same opinion was expressed elsewhere by Abraham (in Witte, 1906 785). Indeed, Einstein's theory did not provide any explanation for variability of mass just like Newton's theory did not provide any explanation of its constancy.

\subsection{The Einstein-Ehrenfest Debate}

A few weeks later, in November of 1906, Sommerfeld wrote to Wien-a supporter of the deformable electron model (Wien, 1904b) - that, after a more careful study, he had become “very impressed" by Einstein's theory 
(Sommerfeld to Wien, Nov. 23, 1906; ASWB Vol. 1, Doc. 102). However, Sommerfeld disabused Wien of his opinion that "Einstein's theory eliminates the electromagnetic mass" (Sommerfeld to Wien, Nov. 23, 1906; ASWB. Vol. 1, Doc. 102). By contrast, he thought that Einstein's mass $\mu$ “[i]n the last $\S^{12}$ can also be of electromagnetic origin". Einstein's point was that "the variability of $\mu$ does not speak against ponderable mass" (Sommerfeld to Wien, Nov. 23, 1906; ASWB Vol. 1, Doc. 102; my emphasis). In general, however, "the foundations of Einstein's theory must be further built before one can treat arbitrary electrons' motions” (Sommerfeld to Wien, Nov. 23, 1906; ASWB Vol. 1, Doc. 102; my emphasis). To Lorentz, Sommerfeld expressed the same surprise that Einstein's theory could reach the same mass velocity dependence by adopting an entirely different "epistemological starting point" (Sommerfeld to Lorentz, Dec. 12, 1906; ASWB Vol. 1, Doc. 103). As we have mentioned, Sommerfeld had written extensively on Abraham's theory, making complicated calculations concerning the behavior of rigid electrons in arbitrary translations and rotations. In spite of all difficulties-as he told to his students in a series of lectures on electron theory that he held in the Winter-Semester 1906/1907-he still firmly believed that "electromagn. foundations of mechanics [was] the Zukunftsmusik" (Sommerfeld, 1906-1907; see Seth, 2010, 31ff.).

\subsubsection{Ehrenfest's Objection and Einstein's Reply}

Planck regarded Einstein's silence toward the nature of mass, shape and charge distribution of the electron as the distinct advantage of the latter's approach. By contrast, Sommerfeld saw in this attitude a way to evade the fundamental question. This opinion was probably widespread among Göttingen electron theorists. In March of 1907, the above mentioned Ehrenfest submitted to the Annalen der Physik a short note (Ehrenfest, 1907) which meant to challenge Einstein's electron-theoretical agnosticism celebrated by Planck (1906c). Ehrenfest might have been convinced by his discussions with Kaufmann (Ehrenfest, 1906c 303, fn.) or by Planck's remark in his paper that one could forgo to introduce a specific 'dynamics of deformation.' It is sufficient to assume that the total energy of the electron is constant, by taking into account the potential energy of the constraining forces. However, Ehrenfest could still not accept Planck's claim that no assumption about the shape of the electron was necessary. Ehrenfest relied on Abraham (1903 174)'s observation that a force-free motion of a rigid extended electron is not possible if the electron is not symmetric. If a three-dimensional ellipsoid moves in uniform translation in a direction oblique to its main axis, the total linear momentum is not parallel to the velocity. Because of the 'equal areas law' (Flächensatz)-that is the conservation of angular momentum-, the angular momentum will change even in translatory motion ${ }^{13}$ A similar issue emerges in the Lorentz-Einstein theory.

"Lorentzian relativity-electrodynamics as it was formulated by Mr. Einstein," Ehrenfest continued, "is considered quite generally as a complete system [abgeschlossenes system]" (Ehrenfest, 1907, 204). Therefore, the theory could not remain silent towards this issue, but it should be able to answer this question "by pure deduction" (Ehrenfest, 1907, 204). The theory does not make any assumption about the shape of the electron at rest. Thus, let us assume that the electron at rest has a non-spherical and non-ellipsoidal shape. The question arises whether a force free-motion of such electron in any direction is possible. If it is not, then the theory should exclude the existence of such electrons "in favor of a new hypothesis" (Ehrenfest, 1907 204). If such motion is indeed possible, then it is necessary to show how the force-free motion of non-symmetric electrons "can be derived from the Einsteinian system, without the use of entirely new axioms" (Ehrenfest, 1907, 205). In a footnote, Ehrenfest also suggested a possible solution of the problem. Since the Lorentz-electron is not stable without introducing "internal deformation forces", one could "give up the (purely electromagnetic) energy- and angular momentum conservation theorem [Flächensatz]" and keep only the center of mass theorem. If the electron is not purely electromagnetic, the torque and momentum due to electrostatic forces could be compensated by the torque and momentum due to the deformation forces ${ }^{14}$

Einstein finished drafting his reply a few weeks later in mid-April. His, counter-objection represents Einstein's first epistemological considerations about the status of the relativity principle that has been preserved and includes the first use of relativity/thermodynamics analogy:

The principle of relativity, or, more exactly, the principle of relativity together with the principle of the constancy of velocity of light, is not to be conceived as a 'complete system,' [abgeschlossenes System] in fact, not as a system at all, but merely as a heuristic principle [ein heuristisches Prinzip] which, when considered by itself, contains only statements about rigid bodies, clocks, and light signals. It is only by requiring [fordert] relations between otherwise seemingly unrelated laws that the theory of relativity provides additional statements. [...] Thus, we are not dealing here at all with a 'system' in which the individual laws are implicitly contained and from which they can be found by deduction alone, but only with a principle that (similar to the second law of the theory of heat) permits the reduction of certain laws to others (Einstein, 1907a 206; my emphasis).

\footnotetext{
${ }^{12} \S 10$ of Einstein, $1905 \mathrm{e}$

${ }^{13}$ Conservation of areal velocity is equivalent to the conservation of angular momentum.

${ }^{14}$ Indeed, Ehrenfest seems to have already realized that the problem was analogous to that of the Trouton-Noble experiment; see Janssen, 19952003
} 
The phrasing of this famous passage contains in nuce some of the essential elements of Einstein's opinion about this matter over the years. The relativity principle, together with the light principle of the velocity of light is to be considered a heuristic principle, like the second principle of thermodynamics. The new kinematics of the rigid body derived from them contains only the transformation equations between space and time coordinates, conceived as measured with rods and clocks synchronized by using electromagnetic signals. To obtain further results, one requires that such formal dependence between spatial and temporal coordinates must be satisfied by some well-tested individual laws of nature (Maxwell field equations, Newton's particle dynamics, etc.). Thus, the relativity principle not only it is not a complete system, as Ehrenfest argued, but it is not even a system containing the individual laws of nature. It is only a principle which demands that otherwise unrelated laws of nature have to satisfy a common condition, i.e., that they should not change their form under a certain set of coordinate transformations. If this is not the case, the laws of nature are not acceptable and must be changed, leading to a new natural law. However, this new law cannot be obtained by pure deduction; one needs an already existing natural law valid in the limiting case as a starting point.

Einstein provided a concrete example of the difference between a 'complete system' and a 'heuristic principle' by giving a brief review of "the theory of the motion of electrons" (Einstein, 1907a 207). In previous electrontheoretical work, when one did not rely on the principle of relativity, in order "to obtain the laws of motion of electrons by electrodynamic methods, one found it necessary to make more specific assumptions on the distribution of electricity so that the problem is not an undetermined one" (Einstein, 1907a 207; my emphasis). As we have seen, a concentration of negative or positive charge is not a stable system. Thus, charge has to be attached to some "(rigid) scaffold" (Einstein, 1907a 207) which is nothing but a small rigid body. detailed hypothesis about the its shape, charge distributions, etc. had to be made to perform the actual calculations. However, Einstein remarked, these assumptions about the structure of the electron were in general quite unsatisfying. The framework corresponds to the introduction of non-electrodynamic forces which balance the electrodynamic ones. Thus, the "laws that govern the motion of such a structure cannot be derived from electrodynamics alone" (Einstein, 1907a 207), against the dictates of the electromagnetic worldview. In this sense, Abraham's model was not better than Lorentz's one.

With the help of the principle of relativity, by contrast, one can proceed in a different way. One starts "from the law for the acceleration of the slowly moving electron (which is assumed or obtained from experience)" (Einstein, 1907a 207; my emphasis), that is Newton's equations of motion, which are supposed to be valid for small velocities. If one knows the mathematical expression of such law respect to the rest system, using eq. [1] and eq. [2] one can obtain, by sheer calculation, "the law for the acceleration of an electron moving at arbitrary speed" (Einstein, $1907 \mathrm{a}$ 207). In this way, as Einstein as showed in the last §10 of his 1905 paper, one obtains the same values of for 'longitudinal' and 'transverse' masses of the moving electron, obtained by Lorentz, without making any assumptions about the structure of the electron. Indeed, as we have see, Einstein idiosyncratically called 'electron' any point-like particles of a certain mass $m$ and arbitrarily small charge $e$. This result is not a direct consequence of the relativity principle obtained by pure deduction; it is an indirect consequence obtained by applying the relativity principle to the already known Newton dynamics of charged point particles.

Einstein did not deny that a theory of the structure of what electron-theorists called 'electron'-that is the elementary particle with a fixed mass $m$ and charge $e-$ was desirable. However, he emphasized that a full treatment was far ahead. Indeed, Einstein seems to indicate that, in principle, Abraham's program based on the classical kinematics and dynamics of the rigid, extended body could be pursued on the base of the new relativistic kinematics and dynamics of the rigid body, if only they were fully developed. Lorentz-Maxwell's electrodynamics is nothing but Maxwell equations together combined with the assumption that the charge is attached to a small framework. If we conceive the framework keeping together the electron as a "rigid body (i.e., one not deformable by external forces), the problem of the motion of the electron can be solved deductively without arbitrariness only if the dynamics of the rigid body is known with sufficient accuracy" (Einstein, 1907a 207; my emphasis). The electron would be simply a microscopic model of a relativistic rigid body with a certain charge distribution, and the motion of the electron will be a certain solution of relativistic of yet-to-be-developed rigid body dynamics, behaving according to Maxwell equations. However, Einstein admitted to Ehrenfest, "[i]f the theory of relativity is correct, we are still far from the latter goal” (Einstein, 1907a 207).

\subsubsection{The Crisis of the Electromechanical Worldview}

This remark was not simply a way to cut the discussion short. Einstein indeed addressed at length the issue of the relativistic dynamics of the rigid body in a paper finished a few weeks later in May (Einstein, 1907d). Relativity theory had only introduced a new kinematics of rigid bodies in uniform parallel translation. Einstein might have already sensed that, because of the relativity of simultaneity, the path towards a kinematic of the rigid bodies, including rotations and accelerations was fraught with pitfalls. The task of developing the corresponding dynamics of extended bodies was probably even more complicated. After having derived a relativistic point particle 
dynamics, as we have seen, (Einstein, 1905b) had already made a first toward a relativistic dynamics of extended rigid bodies in parallel uniform translation that the principle of relativity. He had shown, that in combination with Maxwell's equations, the relativity principle leads to the conclusion that the mass of a body increases or decreases with its energy content. A year later, (Einstein, 1906a), he had extended this result to rigid bodies in which not only mechanical but also electromagnetic processes take place, showing how the center-of-mass theorem could be saved if the electromagnetic energy possesses mass. In his 1907 paper, Einstein (1907d) claimed that he was able to treat only some further special cases of rigid bodies held in equilibrium by opposite external forces that balance each other, i.e., perform no network, but induce a state of stress in the body.

An example might be a charged rigid body in an electromagnetic field. Relying only on the simple fact that forces acting simultaneously from in rest system, do not from the perspective of the moving system, Einstein showed how, differently from classical mechanics, such stresses contribute to the energy and inertia of the rigid body even if they do not perform any net work (Einstein, 1907d). At first sight, Einstein continued, it might seem that we are not far from relativistic dynamics of the rigid motion in a parallel translation. However, in the cases considered, the external forces were constant in time. Analyzing the more general case of variable forces, Einstein started to question the very possibility of a rigid body in relativity theory (Einstein, 1907d). "If relativistic electrodynamics is correct," Einstein admitted, "we are still far from possessing a dynamics of the parallel translation of a rigid body" (Einstein, 1907d 379). Einstein could prove the existence of the "inertia of energy' only by assuming the exact validity of vacuum Maxwell equation from the outset. With this premise, he could treat only some rather special kinds of systems, subject to rather restrictive and unrealistic assumptions. Thus, Einstein conceded, a full "general answer" (Einstein, 1907d 371; my emphasis) to the problem of a relativistic rigid body dynamics "is not yet possible because we do not yet have a complete worldview [Weltbild] that would correspond to the principle of relativity" (Einstein, 1907d 371; my emphasis).

Einstein's caution was the consequence of his conviction that the current worldview, what he called the "electromechanical worldview"-based on Maxwell's equations of the continuous field and Newton's mechanics of discrete particles-was probably bound to be modified (Einstein, 1907d 372; my emphasis). Starting from his analysis of Planck's work on black-body radiation (Planck, $1900 \mathrm{a}$ a c), Einstein had come to realize that a wide range of phenomena remained unexplained within the electromechanical framework. The thermal equilibrium between matter and radiation at Wien's regime (Einstein, 1905d), the laws governing the emission and absorption of radiation (Einstein, 1906d), the anomalous decrease of the specific heats of certain solids with decreasing temperature (Einstein, $1907 \mathrm{~b}$ ). Einstein had obtained these results by calculating the fluctuations of observable macroscopic parameters (temperature, energy, density, etc.) in order to gain information about the micro-structure of matter or radiation. However, he continued, "we do not possess a model [Bild] that corresponds to the requirements mentioned" (Einstein, 1907d 372; my emphasis), that is a proper quantum theory of matter and radiation. In such circumstances, Einstein argued, we can still make use of vacuum Maxwell equations even if we suspect that they are valid only on a macroscopic level. The situation is similar to the case in which we knew "classical theory of thermodynamics" (Einstein, 1905c 550), but not the kinetic theory of heat, that is we did not have a "molecular model [Bildes]" (Einstein, 1907c 569). If we came across the phenomenon of Brownian motion, we would realize that classical thermodynamics and classical mechanics cannot be exactly valid since they both fail to account irregular movements of small particles suspended in a liquid (Renn, 2005). As a consequence, "a radical change of theoretical principles must take place" (Einstein, 1907c 373). Nevertheless, we would continue to use thermodynamics and mechanics in circumstances in which fluctuations are irrelevant.

The same reasoning applies to Maxwell equations that can be used in particular cases, even if, in Einstein's view, they were probably not exactly valid in general. As one can infer from these remarks, Einstein did not believe that relativistic kinematics had any privileged relation to Maxwell's electrodynamics. Maxwell equations happened to satisfy the new kinematics from the outset. Despite their provisional status, Einstein used them in the derivation of the relationship between inertia and energy. However, the gist of Einstein's approach was that his new kinematics of the rigid body in parallel translations had been established relying on relativity and light principle, independently of the validity of any particular individual dynamical laws, including Maxwell's electrodynamics. The new kinematics, as Einstein wrote in response to Ehrenfest, could be compared to experience separately by interpreting space and time coordinates as physically realized in the form of a rigid body of reference and ideal clocks at relative rest synchronized by electromagnetic signals. If we conceive the line spectra of moving positive ions in canal rays as fast-moving ideal clocks, then the relativistic kinematics predicts that we observe the transverse Doppler effect (Einstein, 1907a). This prediction is either observed or not. Thus, one can establish whether the new kinematics is either true or false before any dynamical laws are introduced. Once one gets kinematics right, then one can go on to dynamics. In this context, additional results could be obtained only indirectly, by requiring that well-established dynamical laws of nature-electrodynamics, dynamics of pointparticles, etc.-are consistent with relativistic kinematics. If this was not the case, the law in question should have been changed accordingly. Thus, relativity theory was not a closed system, entailing the individual laws of nature, 
but a sort of open program.

This program appears to correspond to what Planck (1907b), in a paper which he presented in June of 1907 at the Prussian Academy of Science, called a general dynamics of moving, extended systems, which would include the Lorentz covariance not only electrodynamics and mechanics but also thermodynamics (Mosengeil, 1906). Differently from Einstein, Planck gave great importance to the formulation of the laws of nature in terms of the principle of least action (Liu, 1997). In Planck's view, in any branch physics-dynamics point particles, deformable bodies, electrodynamics thermodynamics, etc.-one could construct a kinetic potential $H$ valid for slow velocity. The form of the function which determines the kinetic potential $H$ depends on the particular nature of the system to which the principle of least action is being applied, and it is one of the chief tasks of general physics to discover the form of the function in the various fields of mechanical, electrical and thermodynamic investigation. The special task for the theory of relativity will be to find a general relation applicable to any kind of a system, which shall connect the value of the kinetic potential $H$ as measured with respect to a set of coordinates $K$ with its value $H^{\prime}$ as measured with reference to another set of coordinates $K^{\prime}$ which is in motion relative to $K$. The relativistic laws could be deduced from the classical ones by imposing the invariance of $H=H^{\prime} \sqrt{1-\beta^{2}}$ under coordinate transformations (Liu, 1997). In particular, Planck calculated the kinetic potential $H^{\prime}$ of a volume of blackbody radiation, a system of which he could determined thermodynamic, electrodynamic and mechanical properties, without making any hypothesis about its constitution. In this way Planck could provide an example of rigid body (Planck, 1907a) with non classical inertial properties, whose mass depends not only on velocity and direction, but also on temperature.

As Planck wrote to Einstein in July of 1907, only "a modest tiny group" of physicists had started to work on this new relativistic dynamics (Planck to Einstein, Jun. 7, 1907; CPAE Vol. 5, Doc. 47). Einstein began to correspond with Wien's student Jakob Laub (1907, 1908) and Planck's assistant Max Laue (1907), who visited Einstein in Bern in the Summer of 1907 (Laue to Laub, Sep. 2, 1907; Fölsing, 1993, 211-212) ${ }^{15}$ In July and August 1907, Einstein discussed the problem of superluminal velocities were compatible with Maxwell's equations and electron theory in correspondence with Wien (see CPAE Vol. 5, Docs. 49-53 and 55; see Wien, 1904a), who in turn, had reviewed the matter with Sommerfeld (1907a). In hindsight, one of the most important signs of the impact of the theory was the fact that, at the beginning of October, Minkowski requested to Einstein a copy of his 1905 relativity paper (Minkowski to Einstein, Oct. 9, 1907; CPAE Vol. 5, Doc. 62), which he wanted to discuss at a seminar in electrodynamics with Hilbert in Göttingen in winter term (Pyenson, 1976). In two talks delivered in Göttingen in November (Minkowski, 1907a) and December (Minkowski, 1907b), Minkowski took up the task to modify yet another branch of physics, the electrodynamics of moving media, so that it complies to the relativity postulate. Indeed, Minkowski considered the latter as similar to the principle of conservation of energy. Both principles, he pointed out, are not laws of nature among others, but a new type of law. They posit a demand that all laws of nature, including those that are still unknown, have to satisfy (Minkowski, 1907a Draft RP A, [p. 7]). Since Ehrenfest was at Göttingen at that time, it is not to exclude that he might have read Einstein's response to his objections (Corry, 2004, 220). Minkowski, using different nomenclature, made more explicit what Einstein had only alluded to in his remarks. The relativity postulate is not an insight (Einsicht) into the actual laws of governing matter and fields; it expresses a confidence (Zuversicht) in the universal validity of a requirement that all possible laws of nature have to satisfy (Minkowski, 1907b Draft RP A, [p. 7]; see Corry, 2010 for more details).

\subsection{The Correspondence with Sommerfeld}

In September 1907, Einstein accepted the request of Johannes Stark to write a review article on relativity theory for the Jahrbuch der Radioaktivität und Elektronik, which Einstein completed in early December of 1907. The paper (Einstein, 1908) summarizes the results of a number of his earlier works and incorporates some of the results of the early relativity research. Among the direct consequence of the new relativistic kinematics, Einstein included Laue (1907) and Laub's (1907) derivation of the Fresnel drag coefficient from relativistic addition theorem of velocities (Einstein, 1908 part II). Among the applications of the kinematics to the available dynamical laws, Einstein presented the 'mechanics of the material point' of mass $m$ and charge $e$ that "that we shall call electron" (Einstein, 1908) under the influence of the electromagnetic field. Einstein "used the same method as in my paper cited earlier", but emphasized that "force is defined as in Planck's study [Planck, 1906a]" (Einstein and Laub, $1908 \mathrm{~b}$ ), that is it is defined as the rate of change of momentum. If this definition of force is to be maintained in the theory of relativity, then one has to change the formula for the momentum:

$$
F_{z}=\frac{d}{d t} \frac{m v_{x}}{\sqrt{1-\beta^{2}}}, \quad F_{y}=\frac{d}{d t} \frac{m v_{y}}{\sqrt{1-\beta^{2}}}, \quad F_{z}=\frac{d}{d t} \frac{m v_{z}}{\sqrt{1-\beta^{2}}} .
$$

\footnotetext{
15 "He is a revolutionary", Laue wrote to Laub after the meeting. "In the first two hours of the conversation he overturned all of mechanics and electrodynamics, and this on the basis of statistics” (Laue to Laub, Sep. 2, 1907; Fölsing, 1993 211-212).
} 
With this definition, there is only one mass, which independent of velocity and direction. Thus, Einstein dispensed with the electron theorists' parlance of transverse and longitudinal masses altogether. It is the momentum of a material point of consant rest mass that, differently from classical mechanics, changes with $\mathbf{v}$ in a nonlinear way. Not only does a point particle resist a change more strongly the change of velocity if the rest mass is greater, but also if its velocity is greater. Similarly, the relativity principle imposes a modification of the expression of the kinetic energy of a material point of mass $m$. If two bodies have the same rest mass, the one with the greater kinetic energy resists the action of an external force more strongly. It can be shown that eq. [17] also satisfies the energy law, if $\mathbf{F v}$ is retained as the expression for the work done on the material point per unit time:

$$
F_{x} v_{x}+F_{y} v_{y}+F_{z} v_{z}
$$

and this is found to be equal to

$$
\frac{d}{d t} \frac{m c^{2}}{\sqrt{1-\beta^{2}}} .
$$

Expanding the denominator by the binomial theorem, when $\beta$ is small compared to 1 , the third term is small in comparison with the second term, which corresponds to the old expression for kinetic energy $1 / 2 m v^{2}$. Einstein was now ready to show-although by resorting to some quite artificial examples-that one should grant a real significance to first term $m c^{2}$ which does not depend on velocity, as the expression for the rest energy of the particle (Einstein, 1908 part IV). The energy of a moving mass point does not become zero when its kinetic energy is zero, as is the case in classical mechanics.

By appropriating Planck's approach, Einstein clarified his position in the debate surrounding Kaufmann's experiments. The new relativistic dynamics predicts that, contrary to classical dynamics, the momentum of a particle of constant mass $m$ varies in with velocity according to $\mathrm{G}=\gamma m v$ instead of $\mathrm{G}=m v$. Kaufmann's experiments test the validity of this formula and not variability of mass of the electron (Einstein, 1908, 439ff.). Do projectiles with a velocity approaching that of light resist the action of an external force as predicted by the theory? Since this effect is extremely small, "[a] prospect of comparison with experience", Einstein wrote, "exists only where the moving electrically charged mass points possess velocities whose square is not negligible compared to $c^{2}$ ". Charged mass points of this sort could be found in nature only in "in the cases of the faster cathode rays and the electron rays ( $\beta$-rays) emitted by radioactive substance” (Einstein, 1908439 ). Einstein' discussed in some details Kaufmann measurements of the electric and magnetic deflectability of such rays. Differently from Lorentz (Lorentz to Poincaré, Mar. 8, 1906; SCHAL Vol. 1, Doc. 140), the young Einstein showed surprising confidence that, in spite of Kaufmann's unfavorable results (Einstein, 1908), the relativistic dynamics of the electron was more plausible than the competing "theories of the motion of the electrons of Abraham [1902] and Bucherer [1904]" (Einstein, 1908, 439). The reason that Einstein famously adduced was the velocity-mass formula taken in isolation is not particularly significant. The latter should be considered within a "theoretical systems that encompass larger complexes of phenomena" (Einstein, 1908 439). Qualitative confirmations of the relativistic formula for the momentum, that, so to speak, point in the right direction were sufficiently significant, if considered within a larger groups of experimental results (absence of effects due to the motion of the earth, experiments on the propagation of light in moving bodies, etc.) that seemed to confirm the principle of relativity (Hentschel, 1992).

\subsubsection{Einstein's Letter to Sommerfeld}

Einstein's confidence was not widespread. The Einstein-Planck derivation of the laws of motion of the electrons as structureless point particles continued to appear unconvincing to many of his colleagues. Toward the end of December, Sommerfeld wrote to Lorentz that he was waiting for someone to transform Einstein's stroke of genius into a proper physical theory. He complained about the "inconstruable [unkonstruirbaren] and unintuitive dogmatics” of Einstein's approach (Sommerfeld to Lorentz, Dec. 26, 1907; ASWB Vol. 1, Doc. 115). An English physicist-i.e., one used to work with visualizable models-, he claimed, could not have developed such a theory, which rather expresses "the abstract-conceptual manner of the Semite" (Sommerfeld to Lorentz, Dec. 26, 1907; ASWB Vol. 1, Doc. 115), just like Cohn's phenomenological electrodynamics of moving media (Cohn, 1904a b see Darrigol, 1995a). As he wrote further to Lorentz: "Hopefully you will be able to fill in this genial conceptual skeleton with real physical life” (Sommerfeld to Lorentz, Dec. 26, 1907; ASWB Vol. 1, Doc. 115).

A few days later, Sommerfeld entered in direct correspondence with Einstein, who in his answer mostly emphasized his new work on gravitation (Einstein, 1908 part V) (Einstein to Sommerfeld, Jan. 5, 1908; CPAE Vol. 5, Doc. 72). In particular, Einstein had by that time already realized that any attempt to adapt Newton's action-at-a-distance theory to the framework of special relativity violated the equality of inertial and gravitational mass (see Einstein, 1933). In his reply, Sommerfeld, besides words of admiration for Einstein's theory, must have 
expressed concerns similar to those he had written to Lorentz, as one can infer from Einstein's reply written in mid-January. Einstein was clearly very pleased that a physicist of the stature of Sommerfeld showed interest in the theory, which, he complained, had not provoked the hoped-for reaction. Einstein agreed in principle with Sommerfeld's complaints. However, he justified his approach by resorting again to the comparison between thermodynamics and relativity theory:

\begin{abstract}
First of all, now, the question of whether I consider the relativistic treatment of the mechanics of the electron, as definitive. No, certainly not. It seems to me too that a physical theory can only be satisfactory, if it builds [zusammensetzt] its structures [Gebilder] from elementary foundations [aus elementaren Grundlagen]. The theory of relativity is just ultimately satisfying as, e.g., classical thermodynamics before Boltzmann had interpreted entropy as probability. If the Michelson-Morley experiment had not let the greatest confusion, no one would have accepted the theory of relativity as a (half) salvation. Besides, I believe that we are still far from having satisfactory basic elements for electrical and mechanical processes [Vorgänge]. I am led to this pessimistic viewpoint primarily as a consequence of endless vain attempts to interpret the second universal constant in Planck's radiation law in an intuitive [anschaulich] way. I even seriously doubt that we shall be able to maintain the general validity Maxwell's equations for empty space (Einstein to Sommerfeld, Jan. 14, 1908; CPAE Vol. 5, Doc. 73; my emphasis).
\end{abstract}

In this famous passage, Einstein used for the second time the comparison between relativity theory and thermodynamics to defend his 'mechanics of the electron,' that is the derivation of the velocity-dependence of its mass. A proper theory of the electron was for Einstein an essential task, which, however, could not be solved within the current 'electromechanical worldview.' Einstein was convinced that the known laws governing the electrical and mechanical process were probably not exactly valid. However, Einstein's attempts to modify mechanics and electrodynamics and construct an intuitive model or Bild of these 'quantized' physical systems had failed. Thus, when the negative result of ether-drift experiments had led to 'the greatest confusion' concerning the relations between mechanics and electrodynamics, Einstein preferred to fall back to relativity theory as 'as a (half) salvation.' He relied only on some specific features of mechanics and electrodynamics on which there was overwhelming empirical evidence, like the light and relativity postulate, which do not imply anything about the structure of matter and radiation; he then modified the classical kinematics so that it was compatible with those postulates. In this sense, relativity theory was simply like classical thermodynamics before Boltzmann, which was based on abstract, but empirical motivated principles-like the principle of increase of entropy-which establishes relations between measurable quantities, without introducing a molecular Bild.

It is hard to imagine that Sommerfeld would have found the analogy convincing, considering his negative attitude toward the phenomenological approach to physics (Sommerfeld, 1907b see Seth, 2004 2010, 37ff.). However, Einstein showed to Sommerfeld that the path towards the construction of an electromagnetic model of the electron was full of pitfalls. Sommerfeld probably asked Einstein if a fully electromagnetic electron was possible according to the relativity theory. In principle, Einstein answered, if "one provides an electric charge to a rigid body that is itself massless", one could conceive the mass of the electron as exclusively a mass of an electrostatic energy ${ }^{16}$ According to relativity theory, the mass of the electron at rest could be assumed to be $m=W / c^{2}$, where $E$ is the electrostatic energy: "This holds independently of the shape of the body and the way the charge is distributed" (Einstein to Sommerfeld, Jan. 14, 1908; CPAE, Vol. 5, Doc. 73; my emphasis). However, one cannot set the energy of the moving electron equal to its electromagnetic energy, since stresses exerted by the charges on the framework would contribute to the electron kinetic energy (see Einstein's remark in Szarvassi, 1909 813; CPAE Vol. 2, Doc. 59). However, Einstein did "not like such a conception of the electron to begin with". "[T] he rigid framework with its electrical impregnation arouses my mistrust" (Einstein to Sommerfeld, Jan. 14, 1908; CPAE Vol. 5, Doc. 73). Answering to Ehrenfest, Einstein had pointed out, that, to adequately deal with the problem of the electron, it was necessary to develop a relativistic dynamics of the rigid body. However, Einstein was not sure whether in relativity theory "the abstraction that leads to the concept of the rigid body" was still "appropriate" (Einstein to Sommerfeld, Jan. 14, 1908; CPAE, Vol. 5, Doc. 73).

Concluding his letter to Sommerfeld, Einstein expressed a more radical view. The introduction of a rigid frame to keep the electron together was, in itself, unsatisfying. By contrast, he wrote, "a satisfactory theory should be constituted in such a way that the electron appears as solution" (Einstein to Sommerfeld, Jan. 14, 1908; CPAE Vol. 5, Doc. 73), without introducing the existence of electrons of a certain size and charge by hand. In this way, Einstein believed "that it was not necessary to assume that its electric masses are moving apart" (Einstein to Sommerfeld, Jan. 14, 1908; CPAE Vol. 5, Doc. 73), so that the reason for introducing extraneous stabilizing forces could be eliminated at its root. Such a theory, besides the speed of light $c$, "would have to feature yet another universal constant, owing to the value of which the elementary electric charge has such and such a particular value and no other value" (Einstein to Sommerfeld, Jan. 14, 1908; CPAE Vol. 5, Doc. 73). Einstein concluded he could not fully

${ }^{16}$ (Einstein to Sommerfeld, Jan. 14, 1908; CPAE Vol. 5, Doc. 73). 
justify this opinion at this point. However, it is probable that he had already envisaged the possibility that the two problems discussed in this letter, the issue of the structure of radiation and the problem of the structure of the electron, might have a common root (see below section 2.1.2).

\subsubsection{Behind Sommerfeld's Letter. Abraham's Reaction to Minkowski's Work}

Sommerfeld's letter to Einstein is no longer extant. However, one can get a glimpse of the general attitude of electron theorists toward relativity by considering their reaction to the further development of the relativistic program. In Spring 1908, Minkowski’s December talk (see above section 1.3.2) appeared in the Göttingen Nachrichten under the title Die Grundgleichungen für die elektromagnetischen Vorgänge in bewegten Körpern (Minkowski, 1908). If Einstein had treated relativistic vacuum electrodynamics, a fully relativistic electrodynamics in ponderable matter had not been derived either from phenomenological considerations (Cohn, 1904a b) or from electron theory (Lorentz, 1904). To this purpose, Minkowski introduced two types of vectors with components defined with respect to four spacetime variables treated on equal footing. Several quantities that were separated in the threedimensional vector notation, like the vectors $\mathbf{E}$ and $\mathbf{B}$ turned out to be the components of a single mathematical object, that transform like a vector of type II, $\mathbf{f},{ }^{17}$ which is invariant under Lorentz transformations. By introducing the operator lor (the four-dimensional divergence), Minkowski was able to write Maxwell's equations in a very compact form. In this way, Minkowski succeeded in introducing a formalism such that the mathematical form of the law itself guarantees its invariance under Lorentz transformations. After presenting vacuum electrodynamics in an abstract four-dimensional formalism, Minkowski used it to derive a new phenomenological electrodynamics of moving polarizable and magnetizable media starting from the known electrodynamics at rest as formulated by Hertz. To this purpose, he introduced another vector of type II $\mathbf{F}$ by combining $\mathbf{D}$ and $\mathbf{H}$ for the electromagnetic field in ponderable matter.

The product of the field vectors $\mathrm{f} F$ results into a $4 \times 4$ matrix $\mathbf{T}_{\mathrm{EM}}$ of type II ${ }^{18}$ which expresses the mechanical properties of the electromagnetic field by combining space, energy density $w_{\mathrm{EM}}$, momentum density $\mathbf{g}_{\mathrm{EM}}$, Poynting vector $\mathrm{S}$ and Maxwell stresses $\sigma_{i j}$. Minkowski matrix was not symmetric; this implies that, in ponderable matter, Abraham's relation $\mathrm{S}=c^{2} \mathrm{G}$ does not hold. The lor of this matrix, is a four-vector, $\mathbf{K}$, the force density acting on a charged test particle:

$$
\mathrm{K}=\operatorname{lor} \mathbf{T}_{\mathrm{EM}} .
$$

In an Appendix, by introducing the notion of (Eingezeit) $\tau=t \sqrt{1-\beta^{2}}$, Minkowski used this formalism to carry through the relativistic "reformation of mechanics" (Minkowski, 1908, 13). Like in the case of electrodynamics, he formed a $4 \times 4$ spacetime matrix $\mathbf{T}_{\mathrm{ME}}$ to define the momentum, energy and stresses of an elastic media with the same transformation properties as $\mathrm{T}_{\mathrm{EM}}$. The lor of this matrix expresses the equations of motion of a volume element of constant rest mass density $v$ in analogy with eq. [19] By employing the variational principle in mechanics, Minkowski arrives at the laws for the motion of continuous matter. Minkowski's stipulation of the constant rest mass density turned out to be controversial (section 2.1). However, it simplified Minkowski's derivation of the laws for the motion of a material point of mass $m$, that is to say of an infinitely thin spacetime thread. By restricting his analysis to the case of pure forces, he could defined the driving force $\mathbf{R}$ as the product of the rest mass $m$ and four-acceleration (the derivative of four-velocity with respect $\tau$ ). This allowed Minkowski to write the equations of motion in the following form:

$$
m \frac{d}{d \tau} \frac{d x}{d \tau}=R_{x} \quad m \frac{d}{d \tau} \frac{d y}{d \tau} \quad=R_{y} \quad m \frac{d}{d \tau} \frac{d z}{d \tau}=R_{z} \quad m \frac{d}{d \tau} \frac{d t}{d \tau} \quad=R_{t} .
$$

As it turned out, the relativistic law for the motion of a material point of mass $m$ required four and not three equations, so that the acceleration and force acting on the material point transform as vectors of type I under Lorentz transformations leaving $m$ unchanged. The two aspects of 'force' as 'the time rate of change of momentum' and as 'the space rate of change of energy' are unified in a single concept notion of four-force $\mathrm{R}$. This transformation properties must be shared by all forces in nature if the principle of relativity is to be valid.

As it is well-known, most physicists, including Einstein, did not realize the power of Minkowski's fourdimensional matrix formalism (see e.g., Nordström, 1908). Thus, the discussion initially focused on Minkowski's new physical result, his relativistic electrodynamics of moving media. "Unless I am totally mistaken," Einstein wrote to his wife, "Minkowski's determination of ponderomotive forces is wrong. We now derive the whole thing in another way" (Einstein to Maric, Apr. 17, 1908; CPAE Vol. 5, Doc. 96). A few weeks after the appearance

\footnotetext{
${ }^{17}$ Later a six-vector and today a skew-symmetric second rank tensor, the Faraday tensor.

${ }^{18} \mathrm{~A}$ second rank tensor, which correspond to the stress-energy tensor for the electromagnetic field. The introduction of this mathematical object is a key event in the history relativity theory, to which we cannot doe fully justice here. See Janssen, 2016. Norton, 1992
} 
of Minkowski's treatise, Einstein, in collaboration with Laub (Einstein and Laub, 1908b), re-derived Minkowski'electrodynamics using the old Heaviside-Hertz vectorial notation ${ }^{19}$ However, Einstein and Laub were hostile to Minkowski's phenomenological approach. Einstein regarded sufficient to treat the single electron as a point particle but held necessary to address the problem of the polarizable and magnetizable extended bodies by introducing an "electron-theoretical model [Bild]" (Einstein and Laub, 1908a 541) that is by making some weak assumption of the nature of electric and magnetic dipoles. In particular, Einstein preferred Gilbertian over Amperian magnetic dipoles used by Lorentz.

It is interesting to notice that, on the contrary, Abraham, in the second revised edition of his textbook on electron theory (Abraham, 1908), held the very opposite opinion. Abraham considered Minkowski's phenomenological derivation à la Cohn of the electrodynamics of moving media the most successful application of the relativity principle. The real problems, according to Abraham, arose from the application of the relativity principle to the dynamics of the free electron-as in the case of cathode and Becquerel rays. The latter lead "to unresolved contradictions, if one holds fast to the electromagnetic interpretation that underlies all these developments" (Abraham, 1908 IX; my emphasis). In particular, Abraham, like Sommerfeld, found the relativistic agnosticism toward the shape of the electron untenable (Abraham, 1908). Abraham does not even mention Minkowski's derivation of the relativistic point dynamics, since he probably considered it just as flawed as Einstein's derivation.

Lorentz's original theory, just like in Abraham's own theory, assumes that the electron at rest has a spherical shape. In both theories, it was calculated that the mass/energy relation for the spherical electron at rest was $\mu=4 / 3 W_{\mathrm{EM}} c^{2}$, where $W_{\mathrm{EM}}$ is the electrostatic energy ${ }^{20}$ The difficulties for Lorentz's theory of deformable electron emerges from the electron in motion. If the change in the shape of the electron with the change in velocity is taken into account, work is done in deforming the electron, and the work of the external forces is not equal to the rate of increase of the total electromagnetic energy; thus the values of the energy-derived and momentum-derived longitudinal mass do not coincide. As a consequence a deformable, purely electromagnetic electron does not satisfy the principle of the conservation of energy when set in motion. On the contrary, in Abraham's view, from the perspective of the relativity principle does not seem even possible to construct a stable purely electromagnetic model of the electron at rest. This, was probably what Sommerfeld had in mind when he asked Einstein if, within relativity, the electron could have been thought as fully electromagnetic. As Einstein had replied, for such an electron, the relation between mass and electrostatic energy $W_{\mathrm{EM}}$ should be $\mu=W_{\mathrm{EM}} / c^{2}$ for electrons of any shape and charge distributions. However, electron theorists might have found this result incorrect, since this was not the value one obtains for a purely electromagnetic spherical electron at rest.

As Abraham complained, without the proof that "some rest shape of the electron exists in which the relation $W=m c^{2}$ applies" (Abraham, 1908, 386), the possibility of a fully electrodynamics foundation of the relativistic dynamics of the electron was dubious. However, according to Abraham, "if one refrains at all from an electromagnetic justification of the dynamics of the electron, then every reason for the application of the relativity theorem [...] disappears" (Abraham, 1908 386). Indeed, Abraham, as many at that time, took for granted that the relativity principle was "based on the differential equations of the electromagnetic field" (Abraham, 1908 386; my emphasis) . Ultimately, a relativistic dynamics of the electron without an electromagnetic model of the electron could not be considered acceptable. One can indeed address in this way "many tasks of the dynamics of the electron in which the form of the latter does not play any role" (Abraham, 1908 386), like the radiation emitted by a moving point charge (Abraham, 1904b), e.g. in regions sufficiently far from the charge. However, this is not true in general. Thus, for Abraham, it was after all conceivable "that the postulate of relativity might be valid for the motion of ponderable matter" as treated by Minkowski in a phenomenological way, "but not applicable to the motion of free electrons" (Abraham, 1908, 396).

Indeed, Abraham concluded that it was legitimate to be "skeptical of the application of Lorentz's transformation to a single electron", which anyway was not confirmed by "the measurements of the deflectability of Becquerel rays" (Abraham, 1908, 386). However, relativistic particle dynamics was unexpectedly vindicated just after Abraham had sent the manuscript of his textbook to the publisher in July. At the beginning of September, Bucherer wrote to Einstein about his new experiments using perpendicular electric fields, as suggested by Adolf Bestelmeyer (1907) instead of the parallel electric fields used by Kaufmann. These experiments, he announced, "proved the validity of the relativity principle [Relativprinzip] beyond any doubt" (Bucherer to Einstein, Sep. 7, 19o8; CPAE Vol. 5, Doc. 117). Bucherer already suspected that Kaufmann's data were not fully reliable. However, he claimed, "I was amazed myself when I found the Lorentz-Einstein theory confirmed" (Bucherer to Einstein, Sep. 7, 1908; CPAE Vol. 5, Doc. 117). Bucherer saw no alternative than abandoning his own model of the electron and embrace the Lorentz-Einstein model. Bucherer planned to present his results a few weeks later at the meeting of the Gesellschaft Deutscher Naturforscher und Ärzte (Miller, 1981, sec. 12.4.5). Also, Planck was hoping to meet Einstein there (Planck to Einstein, Sep. 8, 1908; CPAE. Vol. 5, Doc. 118), who, however, exhausted by the double-work

\footnotetext{
${ }^{19}$ see "Einstein And Laub on the Electrodynamics of Moving Media",

${ }^{20}$ See footnote $\overline{6}$
} 
as physicists and patent expert, ultimately decided not to attend. As is well-known, the $8 \mathrm{o}^{\text {th }}$ meeting of the Gesellschaft Deutscher Naturforscher und Ärzte turned out to be a pivotal event in the early history of relativity.

\section{Part 2}

Einstein's relativity/thermodynamics analogy did not achieve the hoped-for result. His derivation of the velocity-dependence of mass by modifying classical point dynamics was still considered unconvincing. Born attempted to construct an electromagnetic model of a relativistically rigid electron that would explain mass-variability. Einstein considered this issue irrelevant. Leaving relativistic research behind, he searched for a non-Maxwellian electrodynamics in which the electron would appear as a solution of non-linear field equations. Both attempts of 'constructing the electron' failed. At the begging of 1911 Laue could prove that Einstein's was right in considering the dynamical properties of the electron that are independent of its constitution. In relativistic dynamics, any closed, extended system in static equilibrium (might be an electron or a planet) has a particle-like behavior. No information about the nature of the electron can be obtained by the experimental verification of relativistic particle dynamics. A consensus was achieved that relativity theory is not a theory but a constraint that all possible theories have to satisfy.

\subsection{Constructing the Electron. The Search for a Relativistic Model of the Electron}

On September 21, 1908, Minkowski (1909b) presented at the Cologne meeting his four-dimensional formalism in a more intuitive diagrammatic form, transforming Einstein's three-dimensional kinematics into the geometry of a four-dimensional manifold, the 'World.' The physical content of relativity theory coincides with the imperative to "undertake a revision of all physics" (Minkowski, $1909 \mathrm{~b} 84$; my emphasis), so that fundamental physical laws do not change under the rotation of the four-dimensional coordinate system to which they are referred. This revision, Minkowski pointed out, has been carried through successfully for questions of thermodynamics and heat radiation (Planck, 1907b); for the electromagnetic processes inside of ponderable bodies (Minkowski, 1908); and for particle mechanics (Einstein, 1905e Planck, 1906c). The "core of the electromagnetic worldview [Kern eines elektromagnetischen Weltbildes]", Minkowski concluded his talk, was nothing but the 'confidence' in the universal of validity of 'world postulate' that was extrapolated from Maxwell equations (Minkowski, 1909b, 88; see Corry, 1998. 2010). If Minkowski deeply transformed relativistic kinematics, the day after (Bucherer, 1908) presented his experimental results, on September 23, Planck (1908a) made a central contribution to relativistic dynamics. He showed, that, to satisfy the relativistic principle of action and reaction, it was necessary to assume that Abraham's proportionality between energy flux and momentum density $\mathrm{S}=c^{2} \mathbf{g}$, applies to any sort of energy current (mechanical, thermal, chemical, etc.). Thus, Planck established the equivalence between the negative mechanical momentum flux in the $x, y, z$ directions and the components of the three-dimensional elastic stress tensor $\sigma_{i j}$. From this point of view, electrodynamics turns out to be, alongside with mechanics, only a special case of the new, yet-to-be-completed, relativistic, general dynamics of moving systems (Planck, 1909 1910a).

The Cologne meeting gave a fundamental contribution in placing the relativity theory at the center of the physics debate (Walter, 19961999 2007). During the discussion that followed Bucherer's (1909) presentation, Minkowski could not hide his joy, for the vindication of the non-rigid 'Parseval electron' over the rigid 'Zeppelin electron' ${ }^{21}$ (in Bucherer et al., 1908 762). The rigid electron appeared to Minkowski as a "monster" that simply did not fit the structure of world which is in the "most intimate harmony" with Maxwell equations (in Bucherer et al., 1908 762). Indeed, in his lecture, Minkowski had presented a diagram in which the contraction of the electron was depicted simply as the geometrical difference between two ways of slicing the world-stripes of "two equal Lorentz electrons" (Minkowski, 1909a 8) with their respective simultaneity hyperplanes. "The time of an electron," so Minkowski described Einstein's contribution, "is as good as the time of any other electron" (Minkowski, 1909a 88). The dynamics of point particles appeared in its most natural form in the four-dimensional formalism. For the first time, Minkowski explicitly defined four-momentum as the product of the four-velocity for the constant mass; the energy and three-momentum of a point-particle transform like components four-dimensional vectors of type I whereas the "mechanical mass $m$ " is constant.

Minkowski showed the advantages of his formalism, by applying it to the description of a field excited by an electron, and the ponderomotive force which is exerted by one moving electron upon another moving electron. In this 'reformed mechanics,' Minkowski, concluded, the "disturbing disharmonies between Newtonian mechanics and the modern electrodynamics disappears by itself" (Minkowski, 1909a 9). Thus, in Minkowski's perspective, "[...] [a]pproaching Maxwell's equation with the concept of a rigid electron" was like going "to a concert with your ears stopped up with cotton wool" (in Bucherer et al., 1908, 762). Minkowski ironically praised "the courage and the energy of the school of the rigid electron", the indefatigable work of Abraham, Sommerfeld, Schwarzschild, etc. (section 1.2.1) who had "jumped the broadest mathematical hurdles in the hope of falling over on experimental physical ground on the other side" (in Bucherer et al., 1908). However, ultimately, all these calculations were an inane attempt to hide the very structure of the 'World.' The rigid electron was "no working hypothesis but a working hindrance" (in Bucherer et al., 1908). Thus, Minkowski's seemed to consider the case of the relativistic dynamics of the electron closed, from a kinematic, dynamical, and experimental point of view. However, the first

\footnotetext{
${ }^{21}$ The reference is to two types of dirigibles.
} 
voices of dissent soon emerged.

\subsubsection{Ehrenfest's and Born's Critique of the Relativistic Treatment of the Electron Problem}

After the Cologne meeting, some of Minkowski's results were at the center of at least three controversies that all involved Planck's relation $\mathbf{g}=c^{2} \mathbf{S}$. (1) Minkowski/Abraham controversy: Abraham considered Minkowski's formulation of the relativistic electrodynamics of moving media suspicious; Minkowski $4 \times 4$ was non-symmetrical in violation of Planck's relation (Abraham, 1909a 1910b). (2) Abraham/Nordström controversy (Nordström, 1909. 1910 Abraham, 1909b 1910a see Liu, 1991): Minkowski's derivation of the particle dynamics was considered incomplete, because it did not consider case of variable rest mass density, as in the case of (Joule heating) that changes the internal energy, and thus, according to Planck's hypothesis mass, but not velocity (Liu, 1991; Norton, 1992 Pyenson, 1979) (3) Born/Ehrenfest controversy: Minkowski's derivation of the particle dynamics was considered insufficient because, Minkowski, like Einstein and Planck, had not provided a suitable model of the electron. As we shall see, a proper reply to this objection also involves the universal validity of Planck's relation $\mathrm{g}=c^{2} \mathrm{~S}$.

In this context, it is worthwhile to consider this last controversy in some details. It shows how Einstein's reply to Ehrenfest by appealing to thermodynamics was not considered convincing by early relativists, who, on the contrary, extended similar objections to Minkowski's derivation of relativistic particle dynamics. Around October of 1908, Ehrenfest, at that time in St. Petersburg, must have written to Minkowski to ask him a copy of his paper 'On Einstein's electrons' (Minkowski to Ehrenfest, Oct. 22, 1908; ESC, 8, Sec. 1, Doc. 24), that is, Minkowski's 'Grundgleichugen' (Minkowski, 1908). Ehrenfest expected to find in Minkowski's paper an electron-theoretical derivation of electrodynamics of moving bodies on which, however Minkowski, was still working. ${ }^{22}$ Minkowski's formulation of the relativistic electrodynamics of moving media, as he himself conceded, was considered at most provisional because, as Minkowski himself conceded, it was not derived from electron theory (Frank, 1908; Minkowski and Born, 1910). However, Ehrenfest was more concerned by Minkowski's treatment of the dynamics of the free electron.

"In Minkowski's work," Ehrenfest wrote in a series of drafts of a letter to his friend, Kaufmann's student Walther Ritz, also a strong critic of relativity (Ritz, 1908a), ${ }^{23}$ "I find just the same incomprehensibility as in Einstein: when I advanced my question, Einstein answered: I have never maintained that my postulate of relativity leads to a full determination-so he wrote literally in his reply in the Annalen" (Ehrenfest to Ritz, undated; ESC 8, Sec. 10, Doc. 528). Minkowski now claims that he did achieve such full determination, but Ehrenfest could not clearly understand why: "I can just repeat my questions to Mr. Minkowsky [sic]: given a resting non-spherical electron can this electron in pure translatory motion (without rumble) if no forces act on it?" (Ehrenfest to Ritz, undated; ESC 8, Sec. 10, Doc. 528). If not, then one has to give up the relativity principle since one could use the rotation of the electron to detect absolute motion; if yes the one has to abandon purely electromagnetic conservation of angular momentum. Ehrenfest admired Minkowski "mathematical-metaphysical construction," which he found more elegant then than the "incomprehensible mollusk-stuff of Einstein" (Ehrenfest to Ritz, undated; ESC 8, Sec. 10, Doc. 528). Nevertheless, Ehrenfest could still not fully understand how "Minkowski without a kinematics of rigidity or deformation gives his electrons stability-tentatively it appears to me that it must necessarily dissolve (for example a rest spherical electron, because of the static electrical repulsion)" (Ehrenfest to Ritz, undated; ESC. 8, Sec. 10, Doc. 528).

Indeed, the advantage of Abraham's rigid kinematic constraints was that one could ignore the stabilizing forces since they do not perform any work. However, it was not clear whether this view could be extended to the relativistic 'electron' or not. As it turned out, Einstein's comparison between relativity theory and thermodynamics did not seem to have had the hoped-for effect on Ehrenfest (Staley, 2008). For Ehrenfest, Minkowski's treatment of the dynamics of the electron left the fundamental question unanswered just like Einstein's. Even Minkowski's new assistant, Max Born expressed similar concerns. After a series of discussions with Minkowski before his sudden death, Born submitted a paper on inertia and relativity (Born, 1909b) in which he made the point quite clear. In Born's view, the Abraham-Sommerfeld theory attempted to deliver an electromagnetic explanation the electron inertia presupposing only the classical kinematic of the rigid body, which, however, violated the relativity

\footnotetext{
${ }^{22}$ Ehrenfest's correspondence has been analyzed by Staley 2008 268ff.). I present here some of it in a different light. Reading Staley, 2008 I realized that Ehrenfest's objections are precisely the ones that induced Einstein's to compare relativity theory and thermodynamics. For this reason, I went back to the original manuscripts and made a partly different selection of passages. In the following, when possible, Staley's translations are used.

${ }^{23}$ The letter is undated but Ehrenfest mentions Ritz, $1908 \mathrm{~b}$ which was finished in October 1908 and published in the same year. Ritz argued that one should save the relativity principle by abandoning Maxwell's electrodynamics in the direction of a ballistic theory of light and taking the retarded potentials as fundamental: "I think, that instead of kinematics, it will be the ether hypothesis, and with it, the representation of phenomena by partial differential equations, that must be abandoned" (Ritz, 1908a 148). As Einstein will later confess to Ehrenfest he had pursued earlier a similar attempt of modifying Maxwell equations to save the relativity postulate. After the failure of this attempt, he probably introduced the light postulate (Martinez, 2004 Norton, 2004).
} 
principle. Relativists, on the contrary, had not provided any satisfying explanation of the inertia electron's inertial mass. "The equations of motion put forward by Einstein, Planck, and Minkowski," Born wrote, "should be seen only as a natural generalization of Newton's equations of motion of the usual mechanics, that are adapted to the electrodynamic; as a consequence, the concept of mass is modified according to the relativity principle, but it is not explained electrodynamically" (Born, 1909b 572; my emphasis). Born felt Einstein, Planck and Minkowski derivation of the variability of the electron was at most a provisional stepping stone. Ultimately, one should explain how the electron self-field produce the variation of mass predicted by the theory.

Born intended to move in the direction of such electromagnetic explanation of inertia in relativity theory which would be comparable to that achieved by the Abraham-Sommerfeld electron model in the absolute theory. Developing Minkowski's derivation of the equations of motion from the Lagrangian formalism (Minkowski, 1908), Born was able to show how "the inertia of a continuously flowing charge can be electromagnetically established" (Born, 1909b 572-573), that is it comes out in the form of a Lagrangian multiplier (Corry, 2004, sec. 4.4). Born (following Levi-Civita, 1908 1909) intentionally renounced to an atomistic view of electricity in spite of the empirical evidence. This choice was motivated by the fact that he was aware that electrons "imagined as rigid bodies, can in no way, be incorporated into the system of electrodynamics built on the principle of relativity, in which no analog is known of a rigid body in arbitrarily accelerated motion" (Born, 1909b 572; my emphasis).

Born's paper appeared in the Annalen at the beginning of March. Just after that, Ehrenfest must have requested an offprint, which Born immediately sent to St. Petersburg (Born to Ehrenfest, Mar. 17, 1909; ESC 1, Sec. 9, Doc. 291). After reading the paper, Ehrenfest probably wrote to Born a letter in which he rehearsed his concerns about the Einstein-Planck-Minkowski approach to the equations of motion. In his long reply, in early July, Born admitted that he could not agree with Ehrenfest's hate toward the relativity principle, although he conceded Einstein had buried "a genial idea" under a load of "mathematical, logical, physical outrages [Schandthaten] and gaucheness [Thatlosigkeiten]” (Born to Ehrenfest, Jul. 5, 1909; ESC 1, Sec. 9, Doc. 292). Ultimately, Born considered Ehrenfest's objections spot on: "In conclusion, you are entirely right: in Einstein, Planck, Minkowski 1) the mass is not electromagnetic, and 2) the electricity is completely structureless. As a consequence, one does not understand what electrons actually are, why, if they exist, they do not explode with an audible crack" (Born to Ehrenfest, Jul. 5, 1909; ESC 1, Sec. 9, Doc. 292). Born, nevertheless, admitted that his derivation was merely a "highly pitiful surrogate for an upstanding, honorable mechanical or electromagnetic mass" (Born to Ehrenfest, Jul. 5, 1909; ESC, 1, Sec. 9, Doc. 292).

"What can be done?", Born concluded somewhat disconsolate (Born to Ehrenfest, Jul. 5, 1909; ESC, 1, Sec. 9, Doc. 292). Born cautiously suggested that a solution could be found in a paper which he had finished in June and was bound to appear in one of the next issues of the Annalen (Born, 1909a). Relativistic kinematics dealt successfully with the accelerated motion of point particles, described by single hyperbolas in Minkowski spacetime. The special relativistic kinematics of extended bodies (described by a group of worldlines) in hyperbolic motion turned out to be more complicated. In particular, in the paper, Born demonstrated that one have to impart to every point of rod a different proper acceleration to assure that the length of the rod does not change. As Born explained to Ehrenfest, the instability of Lorentz's electron that was denounced by Planck and Abraham can be addressed the concepts once one properly define the concepts of "resulting force, resulting momentum, resulting energy (total energy) and the related volume-integrals” (Born to Ehrenfest, Jul. 5, 1909; ESC 1, Sec. 9, Doc. 292). Born suspected that the same issue was behind Ehrenfest's area-law-example see above section 1.3 Once the correct kinematic definition of the rigid electron is introduced, one can develop the dynamics of the electron "and everything is beautiful and easy" (Born to Ehrenfest, Jul. 5, 1909; ESC 1, Sec. 9, Doc. 292). Born was able to derive the equation of motion not simply for charged point particles, but also for "rigid extended electrons," so that for these generalized Newton equations the electromagnetic mass emerged as a consequence (Born to Ehrenfest, Jul. 5, 1909; ESC, 1, Sec. 9, Doc. 292).

However, Born warned Ehrenfest, that, since he had treated only rotation-free motions, he would not "have seen his area-law-objections as refuted" (Born to Ehrenfest, Jul. 5, 1909; ESC 1, Sec. 9, Doc. 292). Indeed, Born postulated that electron had to be symmetric to avoid a turning couple. However, he was confident that "for rotational motions would not give rise to any contradiction" (Born to Ehrenfest, Jul. 5, 1909; ESC 1, Sec. 9, Doc. 292). Born considered his work as a step toward "an electromagnetic foundation of mechanics" (Born to Ehrenfest, Jul. 5, 1909; ESC 1, Sec. 9, Doc. 292; my emphasis), that would achieve for the relativistic rigid electron what Sommerfeld, Hertz, Herglotz, and Schwarzschild had failed to achieve for Abraham's classical electron (Born, 1909c 4). Indeed, Born's electron were rigid just like Abraham's electrons although the definition of rigidity was different. Although, Born could not provide a convincing reason why the electron should have a finite number of degrees of freedom, he was convinced, the new definition of rigidity would ultimately play a central place in the construction of an electromagnetic worldview. Born considered the latter task essential. He regarded a reduction of electrodynamics to mechanics, with its armamentarium of hidden motions and mechanisms, as utterly implausible. Thus, the only reasonable alternative was the reduction of mechanics to electrodynamics. The desirability of such reduction was 
for Born the consequence of "his sense of the unity of nature," which was, "exceptionally developed" (Born to Ehrenfest, Jul. 5, 1909; ESC 1, Sec. 9, Doc. 292).

Born had submitted his paper on the kinematics of the rigid electron (Born, 1909c), dedicated to the memory of Minkowski, on June 13. In it, Born delivered what he had promised to Ehrenfest. Born famously redefined the rigid motion of an extended body with uniform acceleration so that in the co-moving inertial rest frames, the reciprocal distances between the body's points remain constant (see Maltese and Orlando, 1995). Although the new definition of rigidity turned out to be important in itself, Born considered its practical value in the solutions of the fundamental conundrum the dynamics of electrons. Like in Abraham's theory of classical 'rigid' electrons the energy and momentum of an extended electron are obtained by integrating the energy density and the momentum density over the volume that outside of the electron at a moment $t$. In Abraham's theory, in which electrons are 'rigid' in the classical sense, this procedure is justified. However, since the theory does not satisfy the principle of relativity, it led "to extraordinary mathematical complications" (Born, 1909c 4). The series of "voluminous, not to say monstrous papers" (Born, 1956a 251) by Sommerfeld (1904a 1904b 1905), Hertz (1904), Herglotz (1903), Schwarzschild (1903a 1903b 1903c) bear witness to that. In Lorentz's theory, the electron is regarded as deformable in quasi-stationary motion. This theory is compatible with the relativity principle. However, if one defines the total energy and momentum of the electron as spatial integrals for fixed $t$, then one would get entirely different results according in different reference frames. "This circumstance causes the apparent occurrence of a deformation energy and momentum, to which Planck and Abraham have alluded" (Born, 1909a 48).

Born argued that one should carry out the integration over the volume of a properly defined 'relativistic' rigid body which has constant volume in all instantaneous co-moving inertial frames. In this way, the problem of the 'deformation work' emphasized by Abraham and Planck disappears (Born, 1909a 48). The work of the constraining forces is zero, and the rate of increase of the electromagnetic energy is equal to work made by the constant homogeneous electric field in setting the Born rigid electron in uniformly accelerated motion. However, the components of the resulting force perpendicular to the direction of motion do not generally vanish, even in quasi-stationary motion. Yet, this contradicts the observation at cathode- and Becquerel rays, which maintain uniform, rectilinear motion without the influence of external forces. Thus, Born was forced to introduce the additional assumption that the electron must be conceived as a center around which the charge is distributed in concentric layers. Under this assumption, it is necessary to consider only the force component in the direction of motion along the $x$-axis:

$$
-\mu b_{x}
$$

where $b_{x}$ is the $x$ component of the acceleration, $\mu$, the rest mass, is some function of $b$. Born showed that an external constant electric field $E_{x}$ in the direction of motion produces an hyperbolic motion, if the force is defined as $\mu / e b$. For accelerations that vary only slightly from hyperbolic motion, but are arbitrarily large, Born obtained the mechanical equations of motion in Minkowski's form:

$$
\mu \frac{d^{2} x}{d \tau^{2}}=e E_{x} \frac{d t}{d \tau}
$$

where $\tau$ is the proper time (see eq. [20]). For vanishingly small values of $b$ Born found that the value for the rest mass $\mu=4 / 3 W_{\mathrm{EM}} / c^{2}$ were $W_{\mathrm{EM}}$ is the electrostatic energy of the electron. This result "agrees with all other theories" (Born, 1909a 48) ${ }^{24}$ If one instead of the Minkowski mass $\mu$ uses the, usual mass $m$ so that $\mu=m / \sqrt{1-\beta^{2}}$, one can obtain the same equations in Planck's form:

$$
\frac{d m v_{x}}{d t}=e E_{x} \quad \frac{d m}{d t}=\frac{1}{c^{2}} e E_{x} v_{x},
$$

The first equations is the equation of motion in a form that is analogous to Newton's equations of the older mechanics (eq. [17]), and the second one has the form of the energy equation (eq. [18]). The dependency of the mass $m$ on the velocity is given by the Lorentz formula. In this way, Born had recovered Einstein's relativistic particle dynamics, as the consequence of his electromagnetic model of a relativistically rigid, spherical electron. In this sense, Born believed to have completed Einstein's derivation, by providing a dynamical explanation of the variation of mass.

\subsubsection{An Alien to Electrodynamics. Einstein's Model of the Electron}

As we have seen, it was precisely this general skepticism toward Einstein's derivation of the equations of motion of the electron that had prompted to compare it with thermodynamics, both in correspondence with Ehrenfest

\begin{tabular}{l|l|l|l|}
${ }^{24}$ See Fermi, 1921 & 1922. \\
\hline
\end{tabular} 
(section 1.3) and Sommerfeld (section 1.4). Relativity theory, like thermodynamics, could obtain the empirically confirmed velocity dependence of the electron mass, by adapting and modifying Newton's particles dynamics without introducing a model of the electron. Nevertheless, the argument seems to have appealed only to the already converted. Ehrenfest, as we have seen was not convinced, and neither was Born. At the end of 1908, Sommerfeld, by congratulating Lorentz, not without some irony, for the "victory of relativity theory," after Bucherer's results, could not avoid to express nostalgy for the "clarity and causality at the basic physical concepts of your original theory" (Sommerfeld to Lorentz, Nov. 16, 1908; SCHAL Vol. 1, Doc. 177). After all, Einstein himself, had conceded to Sommerfeld a few months earlier, that like thermodynamics before Boltzmann, his derivation of the equation of motion of the electron was only provisional. His opinion about this matter had not changed.

In November of 1908, Bucherer answered to a lost letter of Einstein that he considered "already a great achievement" the fact that in relativity theory "one can calculate everything purely phenomenologically" (Bucherer to Einstein, Nov. 26, 1908; CPAE Vol. 5, Doc. 128; my emphasis). However, he admitted that he shared Einstein's opinion "that one has to search for a model [Bild] that explains all consequences of relativity theory" (Bucherer to Einstein, Nov. 26, 1908; CPAE Vol. 5, Doc. 128; my emphasis). As Einstein had already hinted to Sommerfeld at the beginning of the year, he felt that the theory was not complete, without constructing a proper model of the electron. However, he fundamentally disagreed that it was sensible to proceed by adding a rigid frame that keeps together the electron. By contrast, as he had anticipated to Sommerfeld, he hoped to present the quantum of electricity and quantum of radiation as solutions of non-Maxwellian electrodynamics. Einstein found the opportunity to express his views on the matter in a paper delivered in January and published in March 1909 in the Physikalische Zeitschrift, which was meant to discuss Lorentz's (1908), Ritz's (1908b) and Jeans's (1908) takes on the problem of radiation. As we have seen, Einstein was convinced that not only molecular mechanics did not make justice to thermal properties of matter but also Maxwell-Lorentz's electrodynamics could not be brought into agreement with Planck's radiation formula.

Although Maxwell equations happen to satisfy the new kinematics, the latter is not dependent on the complete truth of that electrodynamics, but only a single result, the source-independence of the velocity of light, that Einstein considered robust enough. On the contrary, Einstein believed that Maxwell-Lorentz electrodynamics was bound to be modified. On the one hand the theory is incompatible with the existence of charges, unless it is supplemented by extraneous theoretical concepts. The elementary quantum of electricity, the electron, as Einstein famously put it, is an "alien to Maxwell-Lorentz electrodynamics" (Einstein, 1909c, 549). External forces of non-electromagnetic nature must be introduced "to construct the electron [Elektron zu konstruieren]"; in particular "usually, one introduces a rigid framework, to prevent the electron's electrical masses from flying apart under the influence of their electric interaction" (Einstein, 1909c 549; my emphasis). Moreover, according to Einstein, Maxwell-Lorentz electrodynamics does not account for the grainy structure of radiation. Vacuum Maxwell equations were only "an intermediary construct," which was not sufficient to "yield a picture [Bild] for a complex of phenomena” (Einstein, 1909c 542). In 1905 Einstein (1905d) had reached this conclusion by using what he called the Boltzmann's principle the relation between entropy $S$ and probability $W$ (see, e.g., Ryckman, 2017. 75 ff., for more details). For those not convinced by this derivation, Einstein introduced a different argument on the Brownian motion exhibited by a mirror moving uniformly through a radiation field. Einstein considered these arguments sufficient to lend support to the claim that the "constitution of radiation must be different from what we currently believe" and present some particle-like behavior (Einstein, 1909c 547).

Maxwell-Lorentz electrodynamics failed on both fronts. Not only was it probably not exactly valid in the immediate neighborhood of concentrated charges, but not even in empty space. Einstein made the bold hypothesis that these two problems were not unrelated (McCormmach, 1970a). The relation $h=W / c$ seems to me to suggest that the same modification of Maxwell electrodynamics that will contain the elementary quantum $e$ as a consequence will also contain the quantum structure of radiation. Einstein conceded that he had not yet succeeded in finding a Lorentz invariant non-Maxwellian electrodynamics that was suited to the "construction [Konstruktion] of the elementary quantum of electricity and the light quantum" (Einstein, 1909c 550). However, he seemed to be optimistic: "The variety of possibilities does not seem so great, however, for one to have to shrink from this task" (Einstein, 1909c 550; my emphasis) ${ }^{25}$ Einstein meant to start from the known wave equation:

$$
D(\varphi)=\frac{1}{c^{2}} \frac{\partial^{2} \varphi}{\partial^{2} t}-\left(\frac{\partial^{2} \varphi}{\partial x^{2}}+\frac{\partial^{2} \varphi}{\partial y^{2}}+\frac{\partial^{2} \varphi}{\partial z^{2}}\right)=0,
$$

This equation, which already contains $c^{2}$ as a coefficient, should be modified so that $e$, possibly $e^{2}$, also appears. The equation or set of equations Einstein was searching for must be homogeneous, but nonlinear, etc., and of course, Lorentz invariant, Given this restrictive conditions, it was worth to try to search for the right equations by trial and error, in the hope some set of equations would deliver the right model of the electron as solution.

\footnotetext{
${ }^{25}$ It is important to keep in mind this turn of phrase. Indeed, Einstein seems to claim that it is valuable to adopt a 'constructive strategy' when the theoretical possibilities are not too many. The 'principle strategy' is by contrast useful to 'restrict the possibilities'.
} 
Einstein sent the paper-a "trifling result of years of reflection"-to Lorentz at the end of March of 1909 (Einstein to Lorentz, Mar. 30, 1909; CPAE Vol. 5, Doc. 146; my emphasis). At about the same time, Sommerfeld admitted to Wien, that he had started to understand what Einstein meant when he claimed that "that $e$ cannot remain an alien to electrodynamics" (Sommerfeld to Wien, Apr. 21, 1909; ASWB Vol. 1, Doc. 150). By contrast, Lorentz was not convinced by Einstein's approach (Lorentz to Einstein, May 6, 1909; CPAE Vol. 5, Doc. 153). Nevertheless, Einstein was confident to be "on the right track" (Einstein to Laub, May 19, 1909; CPAE Vol. 5, Doc. 160). He defended his theory by the return of post, by showing, how it could offer a different take on the fundamental paradox of electron theory (Einstein to Lorentz, May 23, 1909; CPAE Vol. 5, Doc. 163). These partial differential equations, together with their discrete solutions, "could make the rigid framework of the electron unnecessary", and perhaps would be capable of "to construct [zu konstruieren]" not only electrons, but also light quanta, as solutions (Einstein to Lorentz, May 23, 1909; CPAE Vol. 5, Doc. 163).

Einstein exposed his ideas again at the Gesellschaft Deutscher Naturforscher und Arzte in Salzburg in midSeptember (Einstein, 1909a 490) this time revealing the connection between his view of radiations and relativity theory. By eliminating the concept of the ether and showing that a flux of field energy transfers inertial mass, the theory of relativity lends support to the idea that the electromagnetic field and radiation are ultimate irreducible realities and not states of a medium (Einstein, 1909a 490). However, for what the localization of energy is concerned, "the theory of relativity did not change anything" (Einstein, 1909b 820). However, the Boltzmann's principle at least suggests that our current electromagnetic theory cannot correspond to reality in the case of lowintensity radiation. Both of these constraints were sufficient to indicate the task of formulating "a mathematical theory of radiation" (Einstein, 1909b 825) that allows to construct an intuitive model of light quanta as singular points of the radiation field just like electrons are singular points of the electrostatic field. "I am sure it need not be particularly emphasized that no importance should be attached to such a model [Bilde] as long as it has not led to an exact theory" (Einstein, 1909a 500). As Einstein had told Ehrenfest, the relativity principle was not a complete system from which one can obtain a model of the electron by sheer deduction; for the same reason, relativity principle per se was not able to unravel the structure of radiation. However, it could at least serve as a heuristic tool that indicates in which direction the known laws of nature can be modified.

\subsection{The Failure in Constructing a Relativistic Electron Model}

In the same day of Einstein's lecture, on September 22, Born presented at Salzburg his relativistic kinematics of the rigid body as the basis for a dynamics of the electron. Opening the paper, Born explained again why he found Einstein's attitude toward the problem of the electron unsatisfying. Einstein had simply taken for granted the validity of Newton's point dynamics for infinitely slow motions, and, with the help of the relativity principle, he had derived the laws of motion for arbitrary velocity (Born, 1909c 814). In this way, however, Born complained, Einstein did not have provide any "electromagnetic foundation of the inertial effects of the electrons" (Born, 1909c 814), that is, he did not explain how the velocity dependence of mass depends on the electron's self-field. Thus, Born aimed to complete Einstein's work and deliver a proper "new theory of the dynamics of the electron" (Born, 1909c 814) satisfying the relativity principle. As it turned out, Born was still not able to answer Ehrenfest's objection (see above section 2.1.1). A purely electrodynamic Born-rigid electron in uniform acceleration was stable. However, Born conceded that the electromagnetic momentum of his electron was not necessarily always parallel to the velocity.

Born, so to speak, ultimately capitulated to Ehrenfest, and had to make the further assumption that "the charge must be distributed in a specific way, namely in homogeneous, spherical, concentric layers" (Born, 1909c 816; my emphasis), to assure that the electron could move freely in every direction. Thus, the simplest hypothesis about the structure of electron was that it was a Born-rigid body of spherical form at rest. Maxwell's equations apply at the surface of such electron allowing to calculate its dynamics. Sommerfeld, who had presented his work on relativistic velocity addition during the Salzburg meeting (Sommerfeld, 1909), was impressed by Born's talk. Indeed, as he admitted during the discussion, "none could appreciate" Born's efforts better than him, who had "also dealt with the uniformly accelerated motions in the past and, due to the old definition of rigidity", came to "extremely unclear expressions" (in Born, 1909c 816) ${ }^{26}$ However, after the talk, as Born will later recount (see Born, 1910a), Sommerfeld and Einstein immediately realized that a Born rigid body at rest could never be brought into uniform rotation (Einstein to Sommerfeld, Sep. 29, 1909; CPAE Vol. 5, Doc. 279).

\subsubsection{The Failure of Born's Program of Constructing a Rigid Electron}

Ehrenfest (1909) soon reached the same conclusion in a famous paper. As Ehrenfest wrote in a letter to an anonymous friend, possibly Ritz, around the same time: "A month ago I figured out something else also in

\footnotetext{
${ }^{26}$ Sommerfeld refers to his work on electron theory mentioned above in section 1.2 .1
} 
rebus relativibus", something "non-Bornian". "I want you to see it in print, you will laugh about it" (Ehrenfest to 'Friend', undated; ESC ms, Sec. 2, Doc. m59). From the draft of this letter, one can infer that Ehrenfest wanted to show that the construction of a relativistic electron model would have lead to severe difficulties since a rigid electron could not have been brought into rotation. The result would have provided further support to his 1907 Flächensatz-objection. The very possibility of constructing a relativistic rigid electron required a 'Prolegomena to Any Future Relativity Theory of the Rigid Body' (Ehrenfest to 'Friend', undated; ESC ms, Sec. 2, Doc. m59), as Ehrenfest put it with an ironical Kantian touch. Ehrenfest's wish was soon satisfied. Ehrenfest's paper appeared in the November issue of the Physikalische Zeitschrift (Ehrenfest, 1909), together with the contributions to the Salzburg meeting. Already at the beginning of December, Herglotz-a close friend of Ehrenfest from the Wien and Göttingen time-wrote to him (Herglotz to Ehrenfest, Dec. 9, 1909 Herglotz to Ehrenfest, Dec. 16, 1909 Herglotz to Ehrenfest, Jan. 1, 1910; ESC ms, Sec. 2, Doc. m59) that he had finished a paper (Herglotz, 1910) bound to appear on the say issue of the Annalen.

There, he had proved that a body rigid in Born's sense could not have six degrees of freedom as in classical mechanics, but only three. A few days later, Sommerfeld wrote to Wien, that a student of his, Fritz Noether, had nearly finished a paper in which he had obtained independently the same result. "I see that Herglotz expresses himself about his paper in the Annalen," Wien wrote Sommerfeld toward the end of the month: "The Lorentzian relativistic body should be definitively given up. The question is only what should replace it. I hope that here Noether can bring something" (Wien to Sommerfeld, Dec. 27, 1909; DMA. Archiv HS 1977-28/A,369). Noether's (1910) paper appeared in the following issue of the Annalen of which Wien's was co-editor. However, Sommerfeld admitted to Wien that they had not found a solution to the problem: "How a body really behaves (as a rigid body) and how an elastic body behaves, is still not clear to us" (Sommerfeld to Wien, Jan. 16, 1910; ASWB. Vol. 1, Doc. 164).

Neither Herglotz nor Noether directly linked their interests in Born's definition of the rigid body to the dynamics of the electron, which, however, must have been in the back for their mind (see Illy, 1981, 196). Indeed, the fact that a Born rigid electron could not rotate could be seen as a serious challenge to any relativistic electron theory, since one would expect that an electron in a magnetic field rotates. Thus, at the beginning of February of 1910, Born felt compelled to defend his definition of rigidity in a paper that appeared in the March issue of the Physikalische Zeitschrift (Born, 1910a), in which he referred to Ehrenfest's paper and the Einstein-Sommerfeld discussion at Salzburg (Born, 1910a 233; fn. 2). Born agreed that the new concept of rigidity "doesn't contain the necessary six degrees of freedom, it certainly cannot be applied to ordinary material rigid bodies" (Born, 1910a 233). However, he insisted that there was "nothing in the way to employ it as the foundation of the dynamics of the electron" (Born, 1910a 233), i.e., ultimately, of the electromagnetic explanation of its mass (Born, 1910a 233). One could certainly object that the kinematic constitution of the electron is purely hypothetical. However, Born believed to have been so close to the "electromagnetic explanation of the inertial effects of convectively moving electricity" that it was worth pursuing this path in spite of the rotation-issue. There was after all no phenomenon in which the rotation of the electron plays a role; moreover, considering the strange consequences of the electron rotation that Herglotz (1903) and Sommerfeld (1904b) had found in Abraham's theory the fact that the relativistic electron could not rotate could even be seen as an advantage. By contrast, a macroscopic rigid body is not a continuum but is composed of atoms and electrons. In this sense, it is not rigid, but elastic and deformable and thus can rotate (Born, 1910a, 234).

In a note added in the proofs of a paper that appeared in the following April issue of the Physikalische Zeitschrift, Planck expressed disagreement with Born's attempt to limit "the definition of rigidity to a single electron" (Planck, 1910d, 294; fn. 2). It was better to see what results be achieved by applying "the general principles of relativity theory" (Planck, $1910 \mathrm{~d}$ 294; fn. 2) to extended systems in general (see also Planck, 1910c). In pre-relativistic mechanics, after considering the dynamics of particles, it was customary to proceed to a discussion of the dynamics of rigid bodies, and finally to that of deformable bodies. In relativity theory, the intermediate step has to be dropped. If a body is accelerated, it is stressed and deformed. As Planck pointed out, how this happens depends on the development of a relativistic elasticity theory (Planck, 1910d 294). One should then set up the kinetic potential $H$ that agrees with classical elasticity theory at low velocities, and use Lorentz transformations to derive elasticity theory for the general case. Abraham (1910a) soon complained that, in the absence of such a theory, Planck was simply trying to avoid addressing a serious objection against relativity theory (Abraham, 1910a 531). The relativist should show how an elastic body behaves under rotation. Without an appropriate solution of this problem, "Planck's remark cannot be seen a weakening of Ehrenfest's objection” (Abraham, 1910a 531). Abraham (1909b, 1910a) had assumed the point of view of relativity in a controversy with Nordström (1909 1910). However, he was not sure whether relativity would, without a proper notion of rigid body, lead to "a logically permissible [...] representation of the mechanics" (Abraham, 1910a; see also Levi-Civita, 1910). Born (1910b) tried to suggestanother kinematic definition of rigidity with six degrees of freedom, which, however, was usually considered "not much better than the old one" (Sommerfeld to Wien, May 30, 1910; ASWB, Vol. 1, Doc. 168; see 
Ehrenfest, 1910 1128).

Around the beginning of 1910, Ehrenfest seemed to have realized, that Born's project of constructing a purely electromagnetic rigid electron was unnecessary restrictive. "The dynamics of the Lorentz Electron [should be based] not on kinematic but on form-elastic-foundation" (Ehrenfest to Joffe, undated; ESC 3, Sec. 8, Doc. 310). As he pointed out in a letter to his friend Jaffe, at the end of 1909 Abraham (1909b) himself, replying to an objection of Nordström (1909) had already indicated a solution to the electron problem. As Ehrenfest told Jaffe, from Abraham's he had learned that "Planck at the Cologne conference (1908) said that the energy flux that flows through a kinematic connection in relativity theory is connected with a momentum" (Ehrenfest to Joffe, undated; ESC 3, Sec. 8, Doc. 310). The rigid scaffold that holds together the charge concentration is stressed elastically by the pressure of its own electric field which tries to fly apart. The motion of the electron will give rise to an energy current within the material support, which is directed opposite to the motion. According to Planck's hypothesis such current has a momentum density. By taking into account this part of the momentum, many problems of electron theory could be resolved or at least addressed properly (Abraham, 1909b 739). Abraham predictions will turn out to be correct (see below section 2.3). Ehrenfest (see section 1.3.1) had, to certain extent, already outlined a similar approach in his "unfortunate paper" of 1907 : "It annoys me, that there isn't a soul [keine Katz] that is interested in what I say" (Ehrenfest to Joffe, undated; ESC, 3, Sec. 8, Doc. 310).

\subsubsection{The Failure of Einstein's Program of Constructing the Electron as a Solution. Back to Thermodynamics}

In spite of the interest raised by the topic, Einstein confessed to Sommerfeld-who was now fully converted to relativity (Sommerfeld to Lorentz, Jan. 9, 1910; ASWB Vol. 1, Doc. 163)-that he was following the discussion about the rigid body at a distance (Sommerfeld to Einstein, Jan. 19, 1910; CPAE. Vol. 5, Doc. 197). According to Einstein, there were no sufficient empirical data for the construction of a relativistic theory of arbitrarily accelerated bodies. Most of all, Einstein continued to believe that the puzzle of the electron-the scoundrel (Spitzbube) as he ironically called it-should be dealt with by completely abandoning the model of the electron as charge attached to a rigid frame. As in the case of the concept of 'simultaneity' in relativity theory, physicists were held back by a prejudice, "the localization that we give the electromagnetic energy in Maxwell's theory" (Einstein to Sommerfeld, Jan. 19, 1910; CPAE Vol. 5, Doc. 197). Abandoning this prejudice, was more promising than constructing an electron model as a small charged rigid body. "Or is the electron perhaps not to be considered as simple as we believe? There is nothing one cannot come up with when one is an embarrassment" (Einstein to Sommerfeld, Jan. 19, 1910; CPAE Vol. 5, Doc. 197). However, Einstein did not seem to connect the destiny of relativity to the success or failure of 'constructing the electron.' The relativity principle provided at most a "criterion" (Einstein, 1910a 136; my emphasis) to judge the acceptability of a physical theory. Newton dynamics of point particles had to be rejected. The new relativistic dynamics was sufficient to determine the dynamical properties of the electron that are independent of its constitution. The failure to provide a full-fledged electron model was disappointing, but did not have any repercussions on this result. Indeed, Einstein let active relativistic research to "minor prophets" (Born, 1956b 252) and moved on, focusing on his attempt to solve the quantum problem (Einstein to Varićak, Feb. 14, 1910; CPAE Vol. 5[10], Doc. 197a).

As we have seen, Einstein became convinced that without the ether, the old idea of a continuously distributed electromagnetic energy had lost its necessity (Einstein, 1910b). Moreover, in spite of the widespread skepticism (Planck, 1910e: Lorentz, 1910), Einstein believed that the Boltzmann's principle (Einstein, 1910d c e), if applied to radiation, was sufficient to show that the latter must have a structure not given by the ordinary Maxwell's electrodynamics. Although, neither the relativity principle nor the Boltzmann's principle provided any further hint for the positive construction of a model of the granular structure of the electromagnetic field, Einstein was confident that the 'manifold of possibilities' was sufficiently narrow to address the problem directly. "In my lecture in Salzburg, I find nothing for what I think I cannot take responsibility for" (Einstein to Laub, Mar. 16, 1910; CPAE Vol. 5, Doc. 199), he wrote to Laub. Einstein continued to work on the topic during the second half of 1910, convinced that there was "something very fundamental [prinzipielles] behind it" (Einstein to Laub, Aug. 27, 1910; CPAE Vol. 5, Doc. 224). However, by the end of the year, he had to confess that " $\mathrm{t}]$ he solution of the radiation problem has again come to naught" (Einstein to Laub, Nov. 4, 1910; CPAE Vol. 5, Doc. 231). By the beginning of 1911, he had to concede that his plan to attack the quantum problem by modifying Maxwell equations had failed: "With radiation theory, the evil spirit always leads me by the nose" (Einstein to Laub, Jan. 1, 1911; CPAE. Vol. 5[10], Doc. 242a).

The situation was back as it was two years earlier. Planck's radiation law had shown that "our today physical worldview [Weltbild]" rested on the fundamental equations of point mechanics and on Maxwell's equations for the electromagnetic field in a vacuum could not be correct (Einstein, 1911a). Yet, "a proper theory has not yet come into being" (Einstein, 1911a). However, as Einstein emphasized again in a talk given in Zurich in January (before leaving for Prague), what Einstein called for the first time in a title 'relativity theory' was actually not a 'proper theory' like mechanics or electrodynamics. The theory only set up a new kinematics, the latter, once are 
interpreted as measured by rods and clocks is either true or false independently on any particular dynamical law. "The significance of the theory of relativity for physics", Einstein pointed out, was only that it "demands that the mathematical expression of a law of nature valid for arbitrary velocities" (Einstein, 1911c, 13; my emphasis) does not change its form by a certain substitution coordinates. It provides not only a 'criterion' of admissibility of given laws, but also a 'heuristic tool' for obtaining new laws valid for arbitrary velocity "from the laws that are already known for bodies at rest or in slow motion" (Einstein, 1911c 13; my emphasis, 1910a). If we express a natural law mathematically in a system $K$ using the four coordinates $x, y, z, t$, applying the Lorentz transformation and the corresponding transformations rules for natural quantities, one obtains the mathematical expression of the law in a system $K^{\prime}$ with the variables $x^{\prime}, y^{\prime}, z^{\prime}, t^{\prime}$. If the two expressions for $K$ and $K^{\prime}$ are not identical, the law has to be modified acoordingnly. In this way, the relativity principle, "substantially narrows the manifoldness of possibilities" (Einstein, 1911c 13; my emphasis), that is it reduces the number of possible modification of the laws of nature.

During the discussion that followed his Zurich lecture, Einstein clarified this point returning to relativity theory/thermodynamics analogy. Replying to the complaints that the theory was not able to provide a proper Weltbild, Einstein insisted that " $[\mathrm{t}]$ he principle of relativity" is only "a principle [Prinzip] that "narrows the possibilities'; it is not a model [Bild], just as the second law of thermodynamics is not a model" (Einstein, 1911d. $\mathrm{V}$; my emphasis). One can follow two possible strategies to justify the second law of thermodynamics, the law of the limited convertibility of thermal energy, a 'Planckian' or a 'Boltzmannian' one (Einstein, 1910e, 1): (1) "One takes the assumption of the impossibility of a perpetuum mobile of the second kind as the starting point of the argument, then our law appears as almost an immediate consequence of the basic premise of the theory" (Einstein, 1911d VI). (2) However, if "one bases the theory of heat on the equations of motion of molecules, then our law appears as the result of a long series of most subtle arguments" (Einstein, 1911d. VI). Both strategies have "their irrefutable justification" (Einstein, 1911d VI), and correspond to the points of view of Lorentz and of that of relativity theory. In Lorentz's theory the undetectability of ether-drift was deduced from the fundamental equations of the electromagnetic field; Einstein's theory it was postulated as a requirement that any laws of nature have to satisfy (see Lorentz, 1909)

A letter written in March to Vladimir Varičak (Einstein to Varičak, Mar. 3, 1911; CPAE Vol. 5 [10], Doc. 257a) clarifies the motivation behind Einstein's comparison. Varičak had sent to Einstein the draft on a paper (Varićak, 1911) in which-by entering in debate between Vladimir Ignatowski (1910a 1910b) and Ehrenfest (1910 1911)-he claimed that whereas the Lorentz's contraction was real, the relativistic contraction was a psychological effect due to the conventional choice in the definition of simultaneity. Einstein regarded this interpretation as deeply misguided (Einstein, 1911e; see Sauer, 2008) ${ }^{27}$ One can derive the same Lorentz contraction in two ways: as a dynamical "consequence of the modification of the molecular forces" because of the motion through the ether, or, as in the case of relativity, as "kinematical consequences" of new kinematics. "Both points of view are equally legitimate" (Einstein to Varićak, Mar. 3, 1911; CPAE Vol. 5[10], Doc. 257a), and lead to a result that is equally 'real.' To make his point clear, Einstein used as an example the analysis of the derivation of gas dissociation law and its temperature-dependent deviation from the ordinary Gay-Lussac's law relating pressure and temperature.

In his lectures on gas theory, Boltzmann (1896-1898) derived his gas dissociation law "in a molecular-theoretical way" by relying on a model of the gas ${ }^{28}$ To this purpose, he constructed a mechanical model (Boltzmann, 1896-1898. Vol. 2, 206,255f) of the atom, making specific assumptions about the chemical attraction exerted by one atom upon another, the behavior of atoms under collisions, etc. However, Einstein pointed out, one can also proceed in a purely thermodynamic fashion ${ }^{29}$ and derive the "the dissociation law from the second principle [of thermodynamics] without the kinetic theory [of gases]" (Einstein to Varičak, Mar. 3, 1911; CPAE Vol. 5 [10], Doc. 257a). One can derive essentially the same formula by assuming that, in an equilibrium state, when the energy and volume of the gas do not vary, the entropy is at a maximum. Both heuristic stratagems are equally legitimate. In both cases one arrives at the correct law predicting the relative densities of the component vapors as a function of temperature supported by experiment. A 'difference (in principle), Einstein concluded, is not in the "result," but in the, "foundation [Grundlage] on which the research lies" (Einstein to Varičak, Mar. 3, 1911; CPAE Vol. 5 [10], Doc. 257a).

The Einstein-Varičak correspondence seems to put Einstein's relativity theory/thermodynamics analogy in a different light. When Einstein compared relativity theory to 'thermodynamics before Boltzmann' in the letter Sommerfeld (see above section 1.4.1) he did not simply mean to express dissatisfaction for the provisional state of

\footnotetext{
${ }^{27}$ In Einstein's view was one of the major corollaries of the discussion of relativistic rigid body and in particular of Ehrenfest's paradox: "a rotation without elastic deformation according to the theory of relativity is excluded" (Einstein to Varićak, Mar. 3, 1911; CPAE Vol. 5[10], Doc. 257a). This is an objective consequence of relativistic kinematics that cannot be 'transformed away' by any different definition of simultaneity. It is in other terms impossible to change clock's synchronization so that this effect is eliminated (Einstein to Varićak, Mar. 3, 1911; CPAE Vol. 5 [10], Doc. 257a)

${ }^{28}$ Boltzmann, 1896-1898. Vol. 2, Sec. 3.

${ }^{29}$ See Gibbs, $1875^{-1878}$ Einstein had probably Planck's similar result in mind. By celebrating Planck's work in thermodynamics a few years later, Einstein refers in particular Planck, 1887 in which Planck had derived these formulas without making specific microscopic assumptions. See section 3.1 .1
} 
relativity theory, which was incapable of providing a model of the electron. The comparison was probably meant to emphasize how relativity theory, like thermodynamics, without making detailed micro-physical assumptions, was nevertheless able to reach results that have the same degree of 'reality' as those based on electron theory. Having once and for all decided to keep classical kinematics and Newtonian mechanics, Lorentz shifted the explanatory burden on to electrodynamics. Thus, Lorentzian 'relativity' is coextensive with his electrodynamic theory of radiation and matter. On the contrary, Einstein had modified classical kinematics, so that it complied with its two well-stablished empirical facts, without any reference to electrodynamics or to any other theory of matter and radiation. Einstein applied the new kinematics to the existing laws of nature, and obtained thereby a non-Newtonian dynamics for point particles. Conjectures about the nature of molecular forces keeping rods and clocks in equilibrium, the electron's shape, charge distribution, etc. play no role in Einstein's deduction. It is only claimed, that if atoms in canal rays can be taken as fast moving good clocks, then one expects to observe the transverse doppler effect; if electrons in cathode rays can be take, in good approximation, as fast moving charged point particles, then one should observe that their momentum increases non-linearly with velocity. Relativity theory, a this stage, has no means to assure the validity of this conditionals, and had to rely on the available experimental knowledge. However, Einstein considered the latter more trustworthy than electron-theorists speculative conjectures about the structure of matter.

In Einstein's view there was no sufficient evidence that Maxwell equations were exactly valid up to the surface or into the interior of the electron. Einstein even doubted that they were exactly valid in empty space when applied to the radiation field. Thus, Einstein's thermodynamics-like approach had a key advantage. Relativistic kinematics was based on a wealth of empirical evidence summarized in the two postulates, and thus laid on a more secure basis and general basis than any particular theory of matter and radiation, including Maxwell's electrodynamics. As we have seen, Einstein had relentlessly tried to develop a non-Maxwellian electrodynamics as the basis for the construction of electrons and light quanta (section 2.1.2). However, this theory was only one among the possible theories compatible with the relativity principle. The failure of this research program left the relativistic kinematic framework intact. Einstein could have simply moved on to yet another relativistic theory in the hope of constructing a more successful model of the quanta. However, he had become weary of this way of doing physics. Einstein had obtained some significant results using quanta as a "working hypothesis" (Einstein, 1911b XVI), e.g., the theory of specific heats that was confirmed experimentally by Walther Nerst and his collaborators (Einstein to Besso, May 13, 1911; CPAE Vol. 5, Doc. 267; my emphasis; see Barkan, 1999 166ff.). However, when Einstein arrived in Prague-probably at the beginning of April (Illy, 1979)-he progressively abandoned the ambition of delivering a model of the quanta: "I no longer ask whether these quanta really exist," he wrote to Besso, "[n]or am I trying any longer to construct them [zu konstruieren] because I now know that my brain is incapable of prevailing this way" (Einstein to Besso, May 13, 1911; CPAE Vol. 5, Doc. 267; my emphasis). Writing to Laub again in the summer of 1911, he did not mention the quanta anymore but talked only about the "relativistic treatment of gravitation", which was forcing him to abandon the constancy of the velocity of light (Einstein to Laub, Aug. 10, 11; CPAE Vol. 5, Doc. 275)

\subsection{Laue and the End of the Dispute Over the Relativistic Dynamics of The Electron}

Few had taken seriously Einstein's attempt to construct properties of both, electrons and light quanta, as solutions nonlinear theory of the electromagnetic field. However, the difficulties in constructing a relativistic, rigid, purely electromagnetic electron were seen as a serious objection against Einstein's derivation of the velocity dependence of mass. In January of 1911, Laue could point out that Ehrenfest's objection-that had prompted Einstein's relativity/thermodynamics comparison-had ultimately remained without a proper answer. "The dynamics of the mass point," Laue wrote, "was already dealt with by A. Einstein in his first foundational work on the relativity principle, as well as shortly afterward by M. Planck" (Laue, 1911d, 524). The most important result of their investigation were the known formulas for the dependence of longitudinal and transverse mass on velocity, "since then experimentally confirmed at different occasions using the electron" (Laue, 1911d 524 ; my emphasis). However, the electron is not a point-particle. Thus, as Laue pointed out, Ehrenfest, (see section 1.3), could legitimately ask "whether the dynamics of the mass point is still valid for the electron, when one does not ascribe to it-as it ordinarily happens-radial symmetry" (Laue, 1911d 524). Also Born, in constructing his relativistically rigid electron, considered "it necessary to ascribe spherical symmetry to the electron" (Laue, 1911d 524-525). Indeed, only in this way, the transverse components of the electron electromagnetic momentum will sum to zero, giving a resultant momentum parallel to the velocity (section 2.2). Thus, Ehrenfest and Born could claim that, without additional hypothesis about the shape of the electron, Einstein's derivation of the electron dynamics could not be considered complete (section 2.1.1).

Laue's realized that Ehrenfest's objection, as many others that were raised against relativistic dynamics, were ultimately rooted in the difficulties that emerge in extending the relativistic equations which were derived for 
point masses to spatially extended bodies. In classical mechanics, it was customary to start from the dynamics of particles, move to dynamics of rigid bodies, and ultimately address the problem of deformable bodies or the mechanics of continua. In relativity theory, it was initially considered natural to follow the same path. After Einstein and Planck derived relativistic dynamics of point particles, "several authors," Born, Ehrenfest, Herglotz, Noether, etc., "have tried the additional step, to accordingly reshape the dynamics of rigid bodies" (Laue, 1911c 85). However, all these attempts were necessarily doomed to fail. Laue (1911c) could show that in relativity theory, a body not only does not have six degrees of freedom; it does not have any finite number of degrees of freedom as a direct consequence of $c$ as a limiting velocity. One can at most consider the rigid body as the limiting case of a deformable body with a very great elasticity coefficient, as Planck (1910d) had suggested. Thus the problem can be solved "by the theory of elasticity (which is to be adapted to the theory of relativity)" (Laue, 1911c 85). Laue concluded that, in relativity theory, it is more advantageous "to place the dynamics of continua before that of the mass point" (Laue, 1911d 526). The total energy and momentum of point-particles is substituted by the energy and momentum density of extended deformable bodies-fluids or solids as they are treated in hydrodynamics and in the theory of elasticity. The energy and momentum of an extended system are obtained by integrating the energy density and the momentum density over the entire volume.

By considering a point-particle as a special case of an extended system, Laue could show that some "still unsolved problems" (Laue, 1911d 524) in relativistic dynamics could be solved with a single stroke. In particular, Laue addressed several issues, that were related to the Flächensatz, the conservation of angular momentum. By 1911, all the elements necessary to develop the relativistic dynamics where already in place. "From the investigations of Minkowski [1908], Sommerfeld [1910] and Abraham [1909]", Laue pointed out, one can see "that the ponderomotive force [F] related to unit volume (force density) of electrodynamics" can be represented by a four-vector K (Laue, 1911c 528). Furthermore, it was explained by the same authors, that the four-vector is equal minus the fourdivergence $\Delta$ of a world-tensor $\mathrm{T}_{\mathrm{EM}}$ of the electromagnetic field, that, as we have seen, entailed energy, momentum, Maxwell stresses. "Now, Planck [1906] and Einstein [1908] have already announced, that all ponderomotive forces must be transformed by the Lorentz transformation in the same way, as in electrodynamics" (Laue, 1911c 529). Thus, relativity theory requires that, it must be possible, in "all areas of physics, to combine the force density into a four-vector" F (Laue, 1911C 529), which is similarly defined as the four-divergence of a world-tensor T. One can then assume that in any field of physics, there is a world tensor $\mathrm{T}$, whose components have the corresponding meaning as the components of the mentioned electrodynamic tensor. Relying on Sommerfeld (1910); (1910)'s four-dimensional vector calculus, Laue could write the force law and conservations law for energy and momentum in a very compact form:

$$
\mathbf{F}=-\Delta \mathbf{T} \quad \mathbf{F}=-\Delta \mathbf{T}=0
$$

It is convenient to assume that this tensor is symmetric $\left(T_{x l}, T_{y l}, T_{z l}\right)=\left(T_{l x}, T_{l y}, T_{l z}\right)$, to guarantee the conservation of the relativistic angular momentum. The symmetry of $T$ implies the universal validity of Planck's law:

$$
\mathrm{G}=\frac{1}{c^{2}} \mathrm{~S}
$$

With this further requirement, Newtonian particle dynamics based on $\mathbf{f}=$ ma was replaced by relativistic dynamics based on $\mathbf{F}=-\Delta \mathrm{T}$ (see Janssen, 2019). The transformation formulas for momentum, energy density and the stresses $\mathrm{E}$ in the passage from one valid reference system $K$ to another system $K^{\prime}$ is determined by deriving them from the components of the world tensor $\mathrm{T}$ under Lorentz transformations. Integrating energy and momentum density over a finite volume reveals the relations between the rest energy $W$ of the body in $K$ and its energy $W$ and momentum $\mathrm{G}$ in the $K^{\prime}$ in which the body moves at velocity $v$. The expression for momentum had an immediate and important consequence. Even for a body moving in a straight line with unchanging stresses and velocity, relativistic continuum dynamics predicts that the angular momentum is increasing. As a consequence, a torque is necessary to maintain its translatory and uniform motion. Laue could show that a force-free motion is possible only for a particular class of systems which are in static equilibrium in their rest frame without interacting with other bodies. A system of this kind was labeled by Laue a 'complete static system' (Vollständiges statisches System) (Laue, 1911d §5; see Janssen, 2006). If such system is held in equilibrium by opposite external forces, even if such forces do not perform net work, their stresses will also give rise to energy and momentum with respect to a frame in which the system is in motion. Because of eq. [22] this extra contribution exactly cancels the 'unexpected' term $\mathrm{g} \times \mathbf{v}$.

A complete static system is formally characterized by the fact that the volume integrals of the stresses are zero in the rest frame: 


$$
\int s_{i j} d V=0
$$

where $s_{i j}$ is the sum of elastic and Maxwell stresses and $V$ the volume at rest. If one integrates the energy and momentum density of such systems over the volume $V^{\prime}$ of an extended system (where $V^{\prime}=V \sqrt{c^{2}-v^{2}} / c$ ) moving with uniform velocity, then one finds its total energy and momentum:

$$
W^{\prime}=\int w d V^{\prime}=\frac{\sqrt{c^{2}-v^{2}}}{c} \int w d V \quad \mathrm{G}^{\prime}=\int \mathbf{g} d V^{\prime}=\frac{\sqrt{c^{2}-v^{2}}}{\mathfrak{c}} \int \mathbf{g} d V .
$$

For a complete static system the result is much simpler than in the general case:

$$
W^{\prime}=\frac{c}{\sqrt{c^{2}-v^{2}}} W \quad \mathrm{G}^{\prime}=\frac{q}{c \sqrt{c^{2}-v^{2}}} W .
$$

Theses expressions are of course equivalent to eq.[17] and eq.[18]. Thus, the energy, kinetic energy, and momentum of a complete static system (when in uniform or quasi-stationary motion) are as those of a mass point with a mass equal to the rest energy divided by $c^{2}$ :

$$
m=\frac{W}{c^{2}} .
$$

This is usually considered the first actual 'proof' of the mass-energy equivalence (Ohanian, 2008). As a consequence, "the dynamics of the mass point (as it was developed by Einstein and Planck) applies to the case of bodies which are unaffected by external forces, and which are in static equilibrium in their rest system" (Laue, 1911a 1010). This result is "completely independent from other properties of their constitution" (Laue, 1911a 1010). All one needs to know is whether the system is a complete static system and its dynamics is fully determined. The transverse and longitudinal mass of any closed system obey the relativistic formula:

$$
m_{\|}=\frac{\partial G}{\partial v}=\frac{c^{3} m}{\left(\sqrt{c^{2}-v^{2}}\right)^{3}} \quad m_{\perp}=\frac{G}{v}=\frac{c m}{\sqrt{c^{2}-v^{2}}}
$$

Thus, the mechanics of a particle acted on by external forces was derived by Laue as a special case of the relativistic dynamics of a continuous medium, by treating the particle as a complete static system.

The electron at rest and its electric field is nothing but an instance of a physical system in a state of statical equilibrium. This is the only relevant point. A concentration of charge can exist only within a material frame that balances the Maxwell stresses of the Coloumb field. Born felt the need to assume that his rigid electron is spherical because he neglected that "there are stresses of other kinds (which can provisionally be denoted as elastic ones) besides the electromagnetic ones" (Laue, 1911d 542; my emphasis). These elastic stresses will also give rise to energy and momentum with respect to a coordinate system in which the electron is in motion. The energy and momentum coming from the elastic stresses will be equal and opposite to the energy and momentum coming from the electromagnetic stresses. While neither the momentum of the electron's electromagnetic field nor that of the material frame will lie in the direction of its motion, equation section 2.3 assures that the combined momentum will lie parallel to the velocity, whatever the shape of the electron might be. Thus, as it turned out, "Einstein actually answered Ehrenfest's question correctly" (Laue, 1911d 541-542; my emphasis). By imposing the modification of the Newtonian particle dynamics, the theory of relativity enables us to make quite definite statements about the motion of a point charge moving with arbitrary velocity in an electromagnetic field. The velocity dependence of the mass, energy, and momentum follows from the principle of relativity alone, without the need for any assumptions about the shape of the electron. Conversely, from the positive result of Kaufmann-type of experiments on the electrons "one cannot draw conclusions about its form, its charge distribution, and also not as to whether it has another momentum besides its electromagnetic momentum" (Laue, 1911d 541 ). Thus, the very possibility of dynamical electron-theoretical 'explanation' of the mass variability of the electron has been cut at the root. Indeed, Laue's result resolves whatever difficulty might arise for the overall behavior of an electron ${ }^{30}$

Electron-theorists, Laue pointed out in his relativity textbook finished in July, "had no choice" (Laue, 1911b 167; my emphasis). In order to deduce their formulas for the longitudinal and transverse mass of the electron, Lorentz had to construct a suitable electron model (the Lorentz-Poincaré electron model), with an electromagnetic mass

\footnotetext{
${ }^{30}$ Although Laue does not address the question, the same reasoning applies Abraham's objection that the electromagnetic mass of a spherical electron should have is $m=4 / 3 W_{\mathrm{EM}} / c^{2}$, and not $m=W_{\mathrm{EM}} / c^{2}$ as predicted by relativity (section 1.4.2). Also in this case, the problem emerged by neglecting the role of the stresses that keep the electron in equilibrium. The motion of the electron will give rise to an energy flow within the material frame which is directed opposite to the motion. The latter contributes to the total momentum, reducing the $4 / 3$ to 1 , that is to section 2.3 This applies to electrons of whatever shape and charge distribution.
} 
(to account for the mass-velocity dependence), a suitably chosen non-electromagnetic cohesive pressure (to assure its stability), a spherical shape and charge distribution (to avoid the turning couple), etc. By contrast relativity theory could show that the same formulas are satisfied by any closed system constructed using any physical process (mechanical electromagnetic, etc.). The question of the structure of electron remains completely open: "Relativity theory has here still many possibilities" (Laue, 1911b 167; my emphasis). The theory of relativity only limits the range of possible one can construct. A cohesive pressure must be added to make the electron a closed system in static equilibrium in the rest system; however, the question of whether such stresses are mechanical (as in Born's approach) or electromagnetic nature (as, say, in Einstein's non-Maxwellian electrodynamics) remains open. An answer to this question, is an essential task of physics; however, it does not add or subtract nothing to Einstein/Planck/Minkowski derivation of relativistic dynamics. Under the Lorentz transformation the cohesive forces, of whatever nature they might be, must behave like electromagnetic forces. Thus, when they maintain the electron in equilibrium in a rest system, they will do likewise with respect to a moving system. The critique raised against Einstein, Planck, and Minkowski's agnosticism regarding the structure of the electron proved to be entirely unwarranted. Thus, despite the concerns of Ehrenfest, Sommerfeld, and Abraham, it was fully legitimate to do develop the relativistic dynamics of the electron by modifying Newton's point dynamics and adapting it to the relativity principle.

In September 1911, at the meeting of the Gesellschaft Deutscher Naturforscher und Ärzte in Karlsruhe, Sommerfeld could declare that, after only six years, the relativity theory was already a "safe possession of physics" (Sommerfeld, 1911 31). The publication of Laue's (1911b) textbook in the Summer of 1911 probably contributed to settle the dispute over the relativistic particle dynamics and to the general acceptance of relativity theory, more the Einstein's philosophical appeal to thermodynamics. The book popularized Minkowski's rather obscure matrix formalism in the more familiar form of Sommerfeld (1910a)'s four-dimensional vector calculus, presenting the theory in terms of four-vectors, six-vectors, and world-tensors, reintroducing the customary differential operators gradients, divergences, and curls. At the same time, the book clarified the relations between relativity theory and the search for a particular Weltbild, electromagnetic, or of other kinds. The misunderstanding in which the supporters of the electromagnetic worldview had fallen, according to Laue, can be understood by considering the historical development of the electrodynamics of moving bodies.

Since Maxwell's electrodynamics as a theory of the field satisfies the relativity principle, if it is Lorentz invariant, it was assumed that the relativity principle could be saved, if all physics were reduced to Maxwell electrodynamics, if it could be shown that matter itself is ultimately made up of electromagnetic fields. This was, however, a fundamental misunderstanding. Lorentz transformations have been discovered by accident as a formal property of Maxwell equations. However, in principle, they could have been derived from any law of nature, including, say, particle dynamics, if the latter would have been known with sufficient accuracy (Laue, 1913). The relativity principle has a different status than either mechanics or electrodynamics: "the principle of relativity, like the energy principle, stands over all areas of physics (Laue, 1911b 185). It claims to express a criterion for the admissibility of all physical theories" (Laue, 1911b 185f.). The Relativity theory is nothing but a demand, a requirement that we impose to all laws of nature, the requirement that only all forces of nature transform that have vector-character in Minkowski spacetime enter in their formulation (Sommerfeld, 1910a 749).

It is the universal validity of this requirement that transforms it from a "sometimes useful calculation rule" into a powerful physical principle (Laue, 1911b 186). Many branches of physics, including possibly gravitational theory, were in need of updating, according to the general requirement of relativity theory. Thus, after Einstein's successful adaptation of particle mechanics to the relativity principle, the major task became to apply the same strategy to other areas of physics, the laws of which were known for low velocities. The relativistic modifications of available classical electrodynamics or mechanical theories were often a complex technical task that was taken up by a generation of relativists. One should require that solutions to the appropriate dynamical equations of such theories exist that could serve as models of existing physical systems so that theories could be compared with experience. However, the hopes to test the non-classical effects implied by relativistic theories were meager; nevertheless, the fact that the 'relativistisation' of existing theories do not lead to any contradictions provided further confirmation of the viability of the relativistic program ${ }^{31}$ However, it was, so to speak, 'normal science.' The new frontier of physics, as Sommerfeld pointed out in his talk, was the meaning of Planck's energy quantum $h$ (Sommerfeld, 1911). The latter had introduced a fundamentally new element in physics, which was not feasible

\footnotetext{
${ }^{31}$ As we have seen, Planck $1907 \mathrm{a}$ had already modified thermodynamics, Minkowski 1908 had developed a phenomenological relativistic electrodynamics of moving media. Frank (1908), Mirimanoff [1909) and Minkowski and Born (1910) showed how the equations could be also derived from electron theory. Einstein and Laub (1908a 1908b 1909), and Abraham (1909a 1910b) suggested different formulations. In 1911, Herglotz (1911) developed relativistic elasticity, while Paul Epstein (1911) treated the case of static. Attempts to relativistic elasticity theory were made independently by Ignatowski (1911a 1911b), Nordström (1911), and Born (1911). Ernst Lamla (1912a 1912b) developed a relativistic hydrodynamics, Jüttner 1911a 1911b statistical mechanics, etc. The search for a relativistic model of the electron was further pursued, either as Born rigid body (Föppl and Danlell, 1913 Hecke, 1913 Hecke and Behrens, 1912) in which the stresses were assumed to be of mechanical origin, or as a solution of a non-Maxwellian electrodynamics (Mie, 1912a b 1913), in which the stresses were of electromagnetic nature.
} 
either of a mechanical nor an electromagnetic explanation (Sommerfeld, 1911 49). Many were ready to concede that a change in mechanics was necessary. However, probably only Einstein, expected such change to be needed in electrodynamics (Sommerfeld, 1911, 31). Yet, none of those changes were expected to undermine relativistic kinematics which was established independently of either mechanics or electrodynamics.

\section{Part 3}

Einstein had shown that the velocity dependence of the mass, energy, and momentum followed from the modification of Newton's particle dynamics so that it complies with the requirement of relativistic kinematics. Einstein's started to present this result as the application of a more general methodological strategy. Instead of trying directly to modify the existing laws of nature, it is often advisable to search for empirically motivated and mathematically formulated principles that limit the range of possible modifications. New results can be obtained by adapting the existing laws valid in the limiting case to the principle. Einstein's presented his search for a field theory theory of gravitation starting from Newton action-at-a-distance theory as an application of the same strategy. In 1919, these sparse reflections were summarized in the distinction between constructive and principle theories.

\subsection{The First Articulation of Einstein's 'Logic of Discovery'}

At the end of October 1911, a few weeks after Sommerfeld's talk, the major results of quantum theory were discussed at the Solvay Congress in Brussels. However, as Einstein pointed out during the discussion following his lecture on the problem of specific heats in solids, these results were still "not a theory in the usual sense of the word" (Einstein et al., 1912 436, 1914 353). Neither mechanics nor electrodynamics could claim universal validity any longer. However, mindful of the failure of the previous two years, Einstein seems to have become convinced that attacking the quantum problem directly by trying to modify it was not advisable. One should rather resort to the indirect method: "This raises the question of which general laws of physics we can still expect to be valid in the domain with which we are concerned" (Einstein et al., 1912 436, 1914 353; my emphasis); Einstein singled out the energy principle and Boltzmann's principle. The "weak glimmer of theoretical light" had been thrown on the problems involving quanta that had been provided by the use of Boltzmann's principle. Einstein defended again his 'phenomenological' interpretation of the principle as a "relation between quantities that are observable in principle" (Einstein et al., 1912 436, 1914 353; my emphasis). If one interprets Boltzmann's principle in this way, "the equation is either correct or incorrect" independently of "any specific elementary theory (e.g., statistical mechanics)" (Einstein et al., 1912 436, 1914 353). Thus it is possible to use the principle beyond the domain of molecular mechanics in order to draw "conclusions about the admissibility of any fundamental theory whatsoever on the basis of the empirically known thermodynamic properties of a system" (Einstein et al., 1912 436, 1914. 353; my emphasis).

Thus, Einstein seemed to consider advisable to get back the thermodynamics's style of doing physics when the constructive, model-based approach fails (Klein, 1967). In a paper submitted at the beginning of 1912, Einstein (1912c) derived what it remains his most important contribution to quantum theory in that period, the photochemical equivalence law, "in the way of thermodynamics, without quanta" (Einstein to Hopf, Feb. 20, 1912; CPAE, Vol. 5, Doc. 364). In May 1912, by sending to Wien a 'Nachtrag' to this paper (Einstein to Wien, May 11, 1912; CPAE Vol. 5, Doc. 392), Einstein made this point explicit: "I have also come to the opinion, as a result of many fruitless attempts based that through merely constructing [blosses Konstruieren]," one does not arrive at any proper theory of radiation. There were so many possible hypotheses that could be made, that Einstein realized that it was more advantageous to achieve " a new formulation of the question purely thermodynamically, without making use of any model [Bild]" (Einstein to Wien, May 17, 1912; CPAE Vol. 5, Doc. 395; my emphasis). From the failure to construct a model of electrons and light quanta, Einstein seemed to have gained a fundamental methodological lesson. In general it was more suitable to attack the problem indirectly relying on empirically well-confirmed principles. Following the guide of the relativity principle Einstein had derived the empirically confirmed law of motions of free electrons. Einstein's major heuristic guide in his exploration of the quantum structure of matter and radiation had been the Boltzmann's principle, which Einstein had defined without a complete molecular Bild of the system considered. As he wrote to his correspondents, he was now fully immersed into gravitational research in which "even though so few facts are available" (Einstein to Wien, Apr. 11, 1912; CPAE, Vol. 5, Doc. 371), he believed he could rely on the secure guide of a principle, the equality of inertial and gravitational mass Einstein $1912 \mathrm{a}$. 1912b 1912c).

\subsection{1 'The Method of the Pure Theoretician'}

The initial goal was to formulate a special-relativistic theory of gravitation starting from Newton's action-ata-distance theory expressed in the form of the Poisson's equation $\Delta \varphi=4 \pi k \rho$. This enterprise was similar to the change any other field of physics so that it conforms to the new relativistic kinematics (Abraham, 1912b: Nordström, 1912). However, by the time he returned to Zurich in the summer of 1912, Einstein had come to realize that the modification did not go on successfully revealing the limitations of special relativity and ultimately 
burst its framework. Einstein remained as a full professor at the ETH for about two years. With help of his friend Marcel Grossmann, he developed the so-called Entwurf-Theory (Einstein, 1913a), in which both the single gravitational potential and the single a scalar mass-energy density in Poisson's equation for the gravitational field were substituted by multi-component objects (Einstein, 1913a). If the choice of the first object $g_{\mu v}$ was inspired by Gauss's theory of surfaces, the second object, the stress-energy tensor $T_{\mu \nu}$ was the result of Einstein's new appreciation of Laue's continuum dynamics (Einstein, 1914d. [p. 63]; see Rowe, 2008). The methodological approach that Einstein claimed to have followed in this search is described with similar wordings in a technical talk in Vienna (Einstein, 1913c) and in a non-technical one presented in Zurich (Einstein, 1913a).

As Einstein explained, the search for a substitute of Poisson's equation "seemed hopeless at first because of the arbitrariness resulting from the multitude of possibilities" (Einstein, 1913c V; my emphasis). The situation was comparable to that of 19 th century physicists working on electrodynamics. From action-at-a-distance Coulomb's law of electrostatic and the assumption that electrical effects cannot propagate with superluminal velocity, they tried to develop a field-theoretic electrodynamics based on partial differential equations. Of course, it would have been nearly impossible to end up with Maxwell equations, and even less is Minkowski's characterization of the electromagnetic field as a six vector. According to Einstein, the problems faced by theoreticians in gravitational research was analogous. They started with Newton action-at-a-distance theory of gravitation embodied in Poisson's equation and with prohibition of superluminal velocity imposed by special relativity; they sought for a field-based gravitodynamics based on partial differential equations. However, differently than the case of electrodynamics, physicists could rely on far less empirical knowledge of the behavior of fast-moving bodies and strong gravitational fields. If one tries to attack the problem directly, by modifying Poisson's equation, one is lost in a manifold of alternatives. The scalar gravitational potential of Newton's theory could have been substituted by a four-vector, a six-vector, a tensor, etc. Fortunately, Einstein wrote, special relativity not only compels us to modify Newton's theory but it also "limits the confusing manifold of possible generalizations" (Einstein, 1913c. 1250; my emphasis), for the simple fact that forces us to consider four-coordinates at the same level. Then, according to Einstein, " $[\mathrm{t}]$ he theoretical route of march is almost completely given to us if one assumes the general validity of a fundamental empirical law, the equivalence principle" (Einstein, 1913a IV).

This was the fundamental methodological lesson that he had learned in the previous years. Relying on the relativity principle, Einstein, starting from Newton's mass point dynamics valid for low velocities, managed to obtain relativistic particle dynamics that was successfully tested using fast-moving electrons; several other successful modifications of classical theories were obtained by other relativists using the same stratagem. On the contrary, Einstein's attempts to directly modify Maxwell equations, and construct a model of the electron as a solution lead to nothing. Einstein initially believed that the possibilities were not too many, but it turned out not to be the case. In such circumstances, instead of attacking the problem directly by trying to modify the known laws nature, it is often more useful to attack the problem indirectly and search for empirically motivated principles that limit the number of possible choices. In a celebratory paper, Einstein seems to suggest that this was ultimately Planck's "way of working" in his thermodynamics research of the 188os. Einstein called it "perhaps of the method of the pure theoretician in general" (Einstein, 1913b, 1077; my emphasis). One starts out from a proposition of the "greatest possible generality" and deduces from it "individual special results, which can be then compared with experience” (Einstein, 1913b 1077; my emphasis).

This was indeed the heuristic schema that one can see at work in Einstein's relativistic research program. Instead of trying to modify the available theory of radiation, as Ritz (and the young Einstein himself) did, or construct a theory of matter, as Lorentz's did, Einstein realized that it was better to search for empirically motivated principles that any feature theory of matter and radiation would need to satisfy. By adapting available laws of nature to the same requirement, Einstein reached more modest, but more general, results. At the beginning of 1914, before leaving for Berlin, Einstein delivered a lecture (Einstein, 1914f) on the 'problem of relativity.' Einstein explains for the first time the clear presentation of the core structure of this methodological stratagem:

The heuristic value of the relativity theory consists in the fact that it provides a constraint that all of the systems of equations that express general laws of nature must satisfy. All such systems of equations must be constructed such that with the application of a Lorentz transformation they go into a system of equations of the same form (covariance with respect to Lorentz transformations). Minkowski presented a simple mathematical schema to which equation systems must be reducible if they are to behave covariantly with respect to Lorentz transformations. Thereby he achieved the advantage that for the accommodation of the system of equations with the constraint mentioned above it is certainly not necessary to in fact carry out a Lorentz transformation on those systems. From what has been said it clearly follows that relativity theory by no means gives us a tool for deducing previously unknown laws of nature from nothing. It only provides an always applicable criterion that constrains the possibilities; in this respect, it is comparable to the law of energy conservation or the second law of thermodynamics. It follows from a close examination of the most general laws of theoretical physics that Newtonian mechanics must be modified to satisfy the criterion of relativity theory. These altered mechanical equations have proved to be applicable to cathode rays and rays (motion of free electrical particles). Moreover, 
the implementation of the relativity theory has led to neither a logical contradiction nor conflict with empirical results (Einstein, 1914f 340-341; my emphasis).

This passage summarizes in a coherent way the essential point that can be glimpsed in the sparse remarks that we found in his letters and writings starting at least from Spring 1907. Special relativity is not a proper theory. Like the two principles of thermodynamics, it is only "a general criterion of admissibility for a physical theory" (Einstein, 1915a 712) which is already known in approximate form. The advantage of Minkowski's formalism, in Einstein's view, was to provide a "theory of vectors (and its extension, the theory of tensors)" which, in "the search of a new law" allow "inspecting an equation or a system of equations for the purpose of determining, without calculation" (Einstein, 1912-1914 [p. 45]) whether they satisfy this criterion or not. Maxwell equations happen to be already Lorentz invariant; by contrast, Newton's law of motion of point particles had to be modified to satisfy this constraint. In this way, one can obtain laws of motion of rapidly moving material points "without invoking special hypotheses" (Einstein, 1915a 712). This result has no privilege relation to electron theory. However, if one treats the electron as a point charge within the limits of observations, the velocity dependence of mass that the new mechanics implies can be tested by examining the deflection of $\beta$-rays under the influence of electric and magnetic fields. Einstein believed (not without some confirmation bias) that the result was confirmed by Bucherer's (1909) and Hupka's (1909) works.

Einstein could argue that it was legitimate to obtain such result without introducing an electromagnetic model of the electron like thermodynamics obtained the law of dissociation of gases without introducing a mechanical model of the latter. probably Laue's technical proof that convinced even the most irreducible skeptics of the validity of Einstein's procedure, rather than Einstein comparison with thermodynamics. Nevertheless, the successes obtained in this particular case could the validity of the methodological strategy with which it was obtained. The latter could be extended beyond the case of the dynamics of the electron. Of course, Einstein pointed out, the relativity theory does not provide "a universal method that permits the establishment of an absolutely correct theory for a domain of phenomena regardless how little it has been investigated empirically" (Einstein, 1914f 341). The theory only "reduces to a considerable extent the number of empirical observations needed for setting up a theory" (Einstein, 1914f 341-342). This was precisely the advantage of the indirect strategy that Einstein was indispensable in his search for a field-theory of gravitation.

The path from Coulomb's electrostatic to Maxwell's electrodynamics was sufficiently outlined by a large amount of empirical evidence that served as signposts. On the contrary, in moving from Newton's gravitostatics to Einstein's gravitatodynamcs, the maze of possible trails that one could have followed was discouraging. The special relativity principle, by imposing to use all four-variables on the same footing, already provided a first restriction. However, it was not sufficient in itself. "The only way we can reach our goal here is by adding physical hypotheses to what is empirically known in order to complete the basis of the theory" (Einstein, 1914f 341-342). These two requirements combined could mark the trail although the empirical knowledge on which gravitational research could rely was scanty: "This requirement involves a far-reaching restriction on theories of gravitation" (Einstein, 1914f 343; my emphasis). Alternative theories of gravitation could be excluded from the outset, either because they contradicted the relativity principle (Abraham, 1912a c) or the equivalence principle (Abraham, 1912a). Einstein-Grossman-Entwurf-Theory remained as one of the last candidates (Einstein and Fokker, 1914) together with the scalar theory proposed by Nordström (1913a, 1913b).

\subsubsection{Eavesdropping on Nature}

Einstein left Zurich again for Berlin in March 1914. In his inaugural lecture for the Prussian Academy of Sciences July 2, 1914, Einstein attempted for the first time to organize these sparse methodological considerations in a coherent way. "The methodology of the theoretician," he claimed, "mandates implicitly that he uses as his basis general assumptions, so-called principles, from which he can then deduce conclusions" (Einstein, 1914a 740; my emphasis). The deduction of the conclusion is more or less a question of technical ability, whereas for the search of the principles physicists must rely on their gut. The physicist should be able to "eavesdrop [ablauschen] on nature" these general principles by recognizing "in larger sets of experiential facts certain general traits that can then be sharply formulated" (Einstein, 1914a 740; my emphasis), that is in a mathematical precise way. Once the principle has been found "a chain of conclusions sets in, often with unforeseen connections, far transcending the domain of facts from which the principle has been wrested" (Einstein, 1914a 740; my emphasis). In this sense, the principles provided an "aid for the theoretical deduction of the laws of nature", in the sense that they provide a "condition which every general law of nature must satisfy" (Einstein, 1914d). One cannot directly deduce new laws from these principles. One usually starts from well-tested laws whose past success would anyway have to be explained by any new theory as limiting cases. If such laws do not satisfy the mathematical constraint encoded in the principle, they have to be modified so that they do. In this way, the principles restrict the number of possible modifications. 
Thus, Einstein's analogy between relativity and thermodynamics seems to be based on the fact that they are both instances of the same 'logic of discovery' a sort of algorithm for finding new laws given the one that we already known: (a) search for generalizable empirical facts that can be (b) expressed in the form mathematically formulated principles (c) elevate these principles to constraints that all laws of nature have to satisfy if does facts have to hold (d) check whether the known well-established individual laws satisfy this constraint (e) if not, modify them so that they do (f) verify if such modifications imply new predictions that can be tested empirically. There are cases in which we lack suitable principles like in the quantum domain. Planck black-body radiation formula has provided empirical evidence that classical mechanics and possibly electrodynamics could not be exactly valid. However, we have left we no further guide in the search for a new mechanics and a new electrodynamics (Einstein, 1914a 741). There are however cases, like in gravitational research, in which we have "clearly formulated principles" that guided us toward a new field theory of gravitation starting from an action-at-distance-theory valid for weak fields and slow moving particles (Einstein, 1914a 741). However, we do not have the possibility to test the new Entwurf-equations experimentally.

Einstein's faith in the Entwurf-Theory (Einstein, 1914b c) started to crumble toward the end of 1915. Einstein was again lost in the "chaos of possibilities" (Einstein to Hertz, Aug. 22, 1915; CPAE Vol. 8, Doc. 111; my emphasis). It was at this point that the usual heuristic trick kicked in again. In November, Einstein returned to the "demand of general covariance of the field equations", as "a way to limit the possibilities in a natural manner" (Einstein, 1915e 778; my emphasis). Initially, Einstein was convinced that the choice of coordinates had to be further restricted to assure the satisfaction of the conservation laws. This restriction seemed to imply an "admittedly bold additional hypothesis on the structure of matter," that is that the latter would reduce "to purely electrodynamic processes" (Einstein, 1915f g; see, e.g., Janssen and Renn, 2015). However, Einstein soon abandoned this point of view. As is well known, under the pressure of the competition with Hilbert (1915), he arrived at the field equations in November 1915 (Einstein, 1915b c d e). The hypothesis about the electromagnetic structure of matter was substituted with the famous trace term appearing on the right side of the field equations (Einstein, $1915 \mathrm{c}$ ). The requirement of general covariance, Einstein concluded triumphantly, leads "with compelling necessity to a very specific theory of gravitation that also explains the movement of the perihelion of Mercury" (Einstein, 1915c 847). However, the postulate of general relativity "cannot reveal to us anything new and different about the essence of the various processes in nature than what the special theory of relativity taught us already" (Einstein, 1915c 847). In particular the theory does not lend any support to an electromagnetic theory of matter, as Einstein thought for a days. On the contrary, any theory of matter and radiation compatible with special relativity could "be integrated into the theory of general relativity theory, without the latter providing any criteria about the admissibility of such physical theory" (Einstein, 1915c 847; my emphasis).

Thus, in Einstein's in view, the general relativity principle had lead to gravitational field equations, but imposed "no conditions on the structure of matter" (Einstein to Besso, Jan. 3, 1916; CPAE Vol. 8, Doc. 178). The latter was one of the crucial differences respect to Hilbert's approach, who had hoped to combine Einstein and Mie's theory of matter "to obtain the electrons theoretically" (Mie to Hilbert, Feb. 29, 1916; SUB Göttingen Cod. Ms. Hilbert 254/3). Indeed, Hilbert, after discussed explicitly the case of a spherically-symmetric gravitational field and sets up a Lagrangian that would produce an electron solution in this generalized theory. In his review paper published in March 1916, Einstein, without mentioning Hilbert, forcefully emphasized that general relativity was completely silent about these issues. Thus, general relativity was a theory of "gravitation alone", obtained by making "few assumptions about the constitution of matter as possible" (Einstein, 1916b 1111; my emphasis). Concerning the structure of matter, the "general principle of relativity does not indeed afford us a further limitation of possibilities" (Einstein, 1916a 810; my emphasis) respect to special relativity. General relativity simply took Laue's stress-energy tensor $T_{\mu v}$ from special relativity without imposing further modifications We do know that matter consists of charged elementary particles (electrons, positive nuclei). However, question wether such discrete concentrations of charge are held together by gravitational forces, or by forces of other nature remains open. "The general postulate of relativity is unable on principle to tell us anything about this" (Einstein, 1916a 810; my emphasis).

In an unpublished Appendix of the paper (CPAE Vol. 6, Doc. 31), Einstein derived the field equations from a Hamiltonian principle, emphasizing that the Lagrangian of the gravitational field equations of general relativity are almost completely determined by the postulate of general covariance. However, for the choice of the Lagrangian function for matter, such requirement left open "still unassessable many possibilities" (CPAE, Vol. 6, Doc. 31, [p. 3]). Contrary the hopes of Hilbert, general relativity "for what matter is concerned, does not limit the variety of possible choices for the Hamilton function to a higher degree than the postulate of special relativity" (Einstein to Lorentz, Nov. 13, 1916; CPAE, Vol. 8, Doc. 276). As Einstein famously confessed to Hermann Weyl, "Hilbert's assumption about matter appears childish to me"; Einstein refused to mix "the solid considerations originating from the relativity postulate with such bold, unfounded hypotheses about the structure of the electron or matter" (Einstein to Weyl, Nov. 23, 1916; CPAE Vol. 8, Doc. 278). He recognized that the "Hamiltonian function for the construction of electrons [die Konstruktion des Elektrons] represents one of the most important immediate tasks 
of the theory" (Einstein to Weyl, Nov. 23, 1916; CPAE Vol. 8, Doc. 278). However, Hilbert's approach to directly deduce from general relativity a theory of matter was not promising. Einstein was still unclear whether the 'electron' should be conceived as a singularity or whether should be regular solutions of finite dimension (Einstein to Weyl, Jan. 1, 1917; CPAE Vol. 8, Doc. 286). More in general, he was not even confident whether electrons should be taken from the outset as building blocks with a certain size and charge or whether they should appear as discrete solutions of a theory of matter based on continuous fields (Einstein to Dällenbach, Feb. 15, 1917; CPAE Vol. 8, Doc. 278).

\subsection{Toward Einstein's Distinction Between Principle and Constructive Theories}

In December 1916, Einstein signed a contract with the publisher Vieweg for the publication of a popular book on relativity, which was published in Spring 1917. The book was, of course, a good opportunity for making some epistemological considerations. In particular, Einstein insisted in several passages of the booklet that one of the key advantages of special relativity is that it delivers the same results that Lorentz theory had obtained in a "purely electrodynamic way", but without introducing "particular hypotheses as to the electromagnetic structure of matter" (Einstein, 1917a 28). Some of these results were direct consequences of the new kinematics. The young Laue (1907) for instance derived of Fresnel drag coefficient form the relativistic theorem of addition of velocities " without the necessity of drawing on hypotheses as to the physical nature of the liquid" (Einstein, 1917a 35). Further results were the consequence of the indirect, "heuristic power" of relativistic kinematics as a "mathematical condition" imposed the laws of nature (Einstein, $1917 \mathrm{~b}$ §14). The laws of natures that do not satisfy this condition "have to be rejected" and "adapted to the principle of special relativity" (Einstein, 1917a). In particular, classical mechanics "required a modification" (Einstein, 1917a 30), which was confirmed by experiments on the electric and magnetic deflectability of rapidly moving electrons. As we have seen, to account for the same phenomenon, electron-theorists, had to made detailed hypothesis about the structure of the electron. However, these conjectures were ultimately arbitrary, since "electrodynamic theory of itself is unable to give an account of their nature" (Einstein, 1917a 34). Einstein took some pride in emphasizing that relativity theory led to "the same law of motion" found by Lorentz, but "without requiring any special hypothesis whatsoever as to the structure and the behavior of the electron" (Einstein, 1917a, 35; my emphasis).

Contrary to Hilbert's hopes, general relativity did not offer any further insight to solve the problem of the stability of the electron. The great "sagacity [Spürkraft]" of the general principle of relativity, according to Einstein, was again to have provided a "comprehensive limitation which is imposed on the laws of nature" (Einstein, 1917b 67; my emphasis). Indeed, using the principle of general covariance, together with the assumption that the field equations are of the second order, was sufficient to limit the number of possible generalizations of the Poisson's equation for gravity to one possibility, leading Einstein to his great success. Attempts to obtain further results, as Hilbert did, were premature. Einstein's attitude at that time is testified indirectly by the diaries of Rudolf Jakob Humm, a mathematician close to Hilbert's circle in Göttingen. In the summer semester of 1917, he had attended Einstein's lectures on relativity theory in Berlin (see his diary, SzZ, Nachlass R. J. Humm 40-45 [19131977]). According to Humm's reconstruction of a conversation that he had with Einstein, the latter insisted that the general principle of relativity "can give nothing more than the theory of gravitation". He was skeptical that it was possible "to construct a model of world [ein Bild der Welt zu konstruieren]" starting from the relativity principle (see Reichenbächer, 1917). The unification of electricity and gravitation was difficult, since "[ $t]$ he variety of tensor types" or of the corresponding Lagrangians "was far too great and one could not say which should be chosen for the foundation of electrodynamics". Einstein had already first-hand experience how difficult it was to construct "a complete model of world [fertiges Bild der Welt]". "Ten years earlier", he had tried without success "to explain why the electron flies apart" (SzZ, Nachlass R. J. Humm 40-45 [19131977]; see above section 2.1.2).

Thus, in Einstein's view, the general principle of relativity, just like the special principle, was nothing more than "a hypothesis that stringently restricts the possibilities" (Einstein to Mie, Jun. 2, 1917; CPAE. Vol. 8, Doc. 348). Neither principles are however restrictive enough to single out the full-fledged Weltbild physicists are looking for (see Einstein, 1918b). If the general relativity principle guided us to model the gravitational field as a symmetric tensor field, it does not give any clue for why a six-vector represents the electromagnetic field. The existence of elementary particles of certain size and charge remained incomprehensible, etc. It might be against this background, that, possibly as a follow-up to a discussion in person with his friend Heinrich Zangger, Einstein resorted again to the comparison with thermodynamics to clarify the peculiar epistemological status the relativity principle:

I compared the relativity theory to thermodynamics, not with reference to their content but to their method. Both rely on a general principle [Prinzip]:

1. There is no perpetuum mobile 
2. No state of motion is singled out with respect to others

Both derive from the general principles their consequences, without resorting to a model-like theory [modellartigen Theorie], which goes into details. Here lies their reliability, but also their limit (Einstein to Zangger, Aug. 11, 1917; CPAE Vol. 8[10], Doc. 370d)

Einstein continued to consider the comparison between thermodynamics as rhetorically useful to exorcise the apparent uncanniness of the relativity theory. The new, revolutionary relativity theory, at closer inspection, looks very much like old dear thermodynamics everyone is familiar with. They are both theories based on abstract, but empirically grounded principles, that forgo detailed, but speculative microscopic assumptions. Hilbert's attempt to integrate gravitational and electromagnetic theory to derive an electromagnetic theory of matter and construct a model of the electron was the result of a misunderstanding of the nature of the general relativity principle. As a matter a fact, Einstein would soon look with skepticism to the more sophisticated attempts at a unified field theory put forward by Hermann Weyl (1918a), that he considered too speculative and not based on the guide of a physical principle ${ }^{32}$

At this time, Einstein was still annoyed at the insinuation (Lenard, 1918) that, in the path toward general relativity, "speculation allegedly had revealed itself to be superior to empiricism" (Einstein to Besso, Aug. 28, 1918; CPAE Vol. 8, Doc. 607). On the contrary, in Einstein's view the path of the discovery of general relativity teaches once more a general methodological advice: "in order to be reliable, a theory must be built upon generalizable facts" (Einstein to Besso, Aug. 28, 1918; CPAE Vol. 8, Doc. 607). The two principles of thermodynamics are based the impossibility of the perpetuum mobile; special relativity is based on the constancy of the velocity of light which, in turn, "is based on empirical foundations"; the principle the relativity respect to uniform motions is "fact of experience" (Einstein to Besso, Aug. 28, 1918; CPAE, Vol. 8, Doc. 607). General relativity is, as well, is based on an observed fact, the equivalency of inertial and gravitational mass (Einstein to Besso, Aug. 28, 1918; CPAE Vol. 8, Doc. 607). One might complain that these principles alone did not deliver any particular theory. However, as general relativity had shown once again, they turned out to be powerful heuristic tools. As Einstein explains in a semi-popular dialog he submitted toward the end of 1918, the "method of getting to a local field theory for gravitation" was particularly difficult "because one could postulate a large number of theories [gar viele Theorien]" that all agree with the rather limited experience in this field (Einstein, 1918a 701). This embarras de richesse (Einstein, 1918a 701), as Einstein loved to call it, is "one of the most malicious foes making the life of a theoretician difficult" (Einstein, 1918a 701). The principle of relativity "limited these possibilities such that the road which the theory had to go was marked" (Einstein, 1918a 701; my emphasis).

Einstein's attitude toward these issues underwent a 'reorientation' in 1919 (Wünsch, 2005). In Einstein's work seems to emerge a tension between two different approaches. On the one hand, he continued to insist that the principle of general relativity offered "no essential condition for laws of nature but only a point of view for their choice" (Einstein, 1919a [p. 3]). Starting from Poisson's equation this requirement had been sufficiently powerful to lead nearly directly to a field theory of gravitation starting from Newton's action-at-distance theory. However, it did not seem to offer further clues to advance further. On the other hand, Einstein started to make the first attempts to abandon the guide of general relativity principle to account for the stability of the electron. In spite of the "multiplicity of possibilities" he attempted to modify the field equations (with cosmological constant) in order the provide a "construction of matter [Konstrution der Materie]" (Einstein, 1919b 349). The mechanical Poincaré stresses would have been substituted by gravitational stresses. The energy constituting the electron would be so distributed so that $3 / 4$ is to be ascribed to the electromagnetic field and $1 / 4$ to the gravitational field (Einstein, 1919b 355). Between April and May, a correspondence with Theodore Kaluza about is five-dimensional approach might have convinced him that the search for a further unification was less hopeless that he initially thought (Einstein to Kaluza, Apr. 28, 2019; CPAE Vol. 9, Doc. 30, Einstein to Kaluza, May 5, 2019; CPAE| Vol. 9, Doc. 40, Einstein to Kaluza, May 5, 2019; CPAE Vol. 9, Doc. 48; see Wünsch, 2005).

When the results of the British eclipse expedition that took place in those days were made known in November 1919, the interest for the theory rose dramatically. Einstein might have felt compelled to clarify the stance of a theory that had caught the public's imagination between these two different theories, the two relativity theories on the one hand and particular theories of matter and radiation compatible with them, on the other hand. This might be the reason why, when, in November 1919, he was requested to write a popular account of the theory of relativity for the prestigious Times of London, Einstein (1919d) decided to return once again to relativity theory/thermodynamics analogy. Elaborating on the previous sparse remarks we have collected so far, Einstein introduced the now famous distinction between constructive and principle theories. Constructive theories, like the kinetic theory of gases, try to "to construct [zu konstruieren] synthetically a model [ein Bild] of more complex phenomena" (Einstein, 1919c) that behaves according to a particular dynamical law (mechanical model of a gas).

\footnotetext{
${ }^{32}$ In a letter to Seelig from the mid 1950s, Weyl paraphrased what Einstein used to tell him around that time: "Well, Weyl, let us leave it at that! In such a speculative manner, without any guiding physical principle, one cannot make physics" (cit. in Seelig, 1960 274).
} 
Principle theories, like thermodynamics, starting from universally recognized empirical facts (no perpetuum mobile of the second kind) search "analytically" for "mathematically formulated criteria" that any dynamical law must satisfy if those facts have to hold (second principle of thermodynamics) (Einstein, 1919c).

I do not linger further on these well-known passages. The reader might recognize the same language that Einstein had used often in the past, a language that was familiar to any physicist of his generation. Today, scholars have read in this passage Einstein's deep philosophical dissatisfaction for the lack of explanatory power of the relativity theory as theory of principles (Brown, 2005). Others have pointed out that the principle/constructive theories opposition ultimately disarmingly unoriginal (Frisch, 2005). Probably, however, both camps measure this little article against the wrong standard. Einstein did not aim to provide the readers of the London Times with a path-breaking epistemological treatise compressed in two newspaper columns. The motivation behind Einstein's choice of topic was far more modest. It can be glimpsed in the opening paragraph of a more technical presentation of the theory that he probably wrote a few weeks later. Einstein revealed that, after the confirmation of general relativity through the eclipse observations, he was often asked to give a popular presentation of the theory. Einstein was quite glad to comply. He perceived "a certain danger" that the "rather complicated mathematical form of the theory threatens to overshadow its simple (and natural) physical content" (Einstein, $192 \mathrm{ob}$ [p. 1]). The highly mathematical form is merely a tool, while the theory was based on "(general) simple principles to which physical experience has led us" (Einstein, 1920b [p. 1]; my emphasis). The comparison relativity theory/thermodynamics served precisely to make this point. In spite of their apparent abstractness, the relativity principle, like the second principle of thermodynamics, has "no speculative origin, it rather owes its discovery only to the desire to adapt theoretical physics to observable facts as closely as possible" (Einstein, 1921a [p. 1]; my emphasis).

The London Times was probably written with this reassuring intention. However, behind the characterization of the relativity theory as a principle theory, there was not only a negative, defensive strategy. Einstein was also offering a testimony of the style of doing physics that "he enjoyed the most" (CPAE Vol. 9, 993, early 1920) based on the capacity of singling out few general principles from which a cascade of results can be derived (Einstein, 1920a), rather than proceeding with brute mathematical force solving separate problems. Thus, the constructive/principle distinction, somewhat unwittingly, also provides us with a positive insight into what we have called Einstein's 'logic of discovery,' the heuristic stratagem that was at bases of many of his successes, including his last gravitational theory. Einstein was fully aware that without the guidance of a principle would have been extremely challenging to achieve comparable results: "Once one renounces to the relativity principle and a the same time to the essential unity of inertial and ponderable mass", Einstein wrote to a correspondent, one is lost in "a hopeless manifold of possibilities" (Einstein to Petzold, Jul. 21, 1920; CPAE Vol. 10, Doc. 80). Einstein was aware that moving in the direction of a unified field theory (Einstein, 1921b) capable at the same time delivering a theory of matter (Einstein to Lorentz, Feb. 22, 1921; CPAE Vol. 12, Doc. 57), he was undertaking a slippery road.

After exploring again Kaluza's (1921) five-dimensional formalism without success (Einstein and Grommer, 1923), Einstein seems to have become disillusioned. All other existing alternatives (Eddington, 1921 Weyl, 1921) seemed to him hopelessly speculative. As he wrote to Weyl, "in order to really advance, we must again find a general principle eavesdropped on nature [ein allgemeines, der Natur abgelauschtes Prinzip]" (Einstein to Weyl, Jun. 6, 1922; CPAE Vol. 13, Doc. 219), something comparable to the equivalence principle in general relativity. As the reader might recall, Einstein had used the same turn of phrase at his arrival in Berlin, in his inaugural lecture, when he was in the middle of his search of general-relativistic field equations (see section 3.1.2). At this point, however, Einstein had to concede that the voice of nature had become too faint and no further principle could be overheard. Nevertheless, the 'double dualism' (between two fields and between matter and field) that characterized the current state of physics was unbearable to him. The general relativity principle imposed the search for a field theory. However, it did not offer further restrictions. Thus, Einstein was lost again into the 'chaos of possibilities,' driven only by his quasi-religious faith in the rational structure of reality (Dongen, 2010 , see Giovanelli, 2018a).

Einstein could only proceed by trial and error. He initially embraced (Eddington, 1921)'s purely affine approach (Einstein, 1923a b c), later moved to the metric-affine approach (Einstein, 1925a), returned to the trace-free equations after a correspondence with G. Y. Rainich (Einstein, 1927a), and then, inspired by the work of O. Klein, moved to the five-dimensional approach (Einstein, $1927 \mathrm{~b}$ c). In none of these cases the choice of mathematical structure to represent the combined electromagnetic/gravitational field was empirically motivated from the outset as in case of his theory of gravitation; the comparison with experience was possible, so to say, only at end, by integrating the field equations and finding solutions that can serve as a model of the electron. The moderate empiricism of the young Einstein, which relied on empirically motivated and mathematically formulated principles that limit the freedom of theorizing, was progressively substituted by a form of extreme rationalism, by the conviction that mathematical simplicity was the only criterion of theory choice (see Giovanelli, 2018b). 
However, Einstein was well aware that the relativity theory in itself had a peculiar status with respect to any general-relativistic constructive field theories, not only the final theory of the complete field he was searching for, but also with respect to the theory of the isolated gravitational field he had already found. Einstein explained his point of view with particular clarity in a little known passage of a book review (Einstein, 1928; see Giovanelli, 2018 b for further details). Relativity theory, Einstein pointed out, did not deliver a "new system of physics" (Einstein, 1928, 163), it is ultimately not a new 'theory.' On the contrary, starting from the "experiences with light, inertia, and gravitation" it only introduced a new "formal principle that the equations of physics ought to [satisfy]" (Einstein, 1928 163; my emphasis). In particular, the relativity principle requires that the equations of physics "ought" to be covariant with regard to any arbitrary substitution of coordinates (Einstein, 1928 163). This, however, Einstein continued, "would be much too narrow a base on which to erect the edifice of theoretical physics" (Einstein, 1928, 163). Relativity theory achieves physically significant results by "adapt[ing] the basic laws of physics-as they were known before-to this principle with as few changes as possible" (Einstein, 1928 163; my emphasis). This was the cornerstone of Einstein's 'logic of discovery.' Just like the velocity-dependence of mass was obtained by adapting Newton's particle dynamics to the special relativity principle, the field theory of gravitation was obtained by adapting Newton's scalar theory of gravitation to the general relativity principle. Thus, not the simple enunciation of the principle is the content of the theory, but "the adaptation to the principle of relativity" (Einstein, 1928 163). One should speak of the relativity theory as "physics adapted to the principle of relativity" rather than of "a new system of physics" (Einstein, 1928 163; my emphasis).

\section{Part 4}

Einstein started to use the relativity theory/thermodynamics comparison not merely as a post hoc justification but as the description of the path he had followed in setting up special relativity. In particular, Einstein insisted that he was motivated by his skepticism toward Maxwell equations. The Lorentz transformations are not the by-product of Maxwell equations, or any particular dynamical law. Like the principles of thermodynamics, they were derived from well-established empirical facts. For this reason, they could be elevated to a constraint that all laws of nature have to satisfy. Additional results are obtained by modifying the existing laws of nature valid in the limiting case so that they satisfy this constraint.

\subsection{A Retrospective look. Out of Einstein's Later Years}

In September 1933, Einstein left Europe and settled permanently in the United States in October, taking on the position of professor at the Institute for Advanced Study in Princeton. At the end of December of 1934, in one of his first public appearances in his newly adopted country, Einstein delivered the Gibbs lecture in Pittsburg. They were published the following year (Einstein, 1935). The paper was an attempt to provide a new proof for the mass-energy equivalence that would utilize only mechanics, avoiding any reference to electrodynamics. Special relativity, Einstein pointed out in the opening paragraph, emerged out of the Maxwell electrodynamics. As a consequence, even in the derivation of the mechanical concepts, the electrodynamic consideration played an essential role. However, at closer inspection, this connection between relativity theory and electrodynamics was accidental: "The Lorentz transformation, the real basis of the special relativity theory in itself, has nothing to do with the Maxwell theory" (Einstein, 1935, 223). As we shall see, this point of view will be repeated in different forms in many writings of Einstein's American years. Lorentz and Poincaré uncovered the Lorentz transformations as a formal property that Maxwell equations equations happen to satisfy. Einstein, on the contrary, doubted that "the energy concepts of the Maxwell theory [could] be maintained in the face of the data of molecular physics" (Einstein, 1935 223). Thus he derived the Lorentz transformation from well-confirmed empirical facts and elevated them to a fundamental requirement that any fundamental law of nature must satisfy. In this sense, the content of special relativity could be "summarized in one sentence", which has the characteristic form of an imperative: "all natural laws must be so conditioned that they are covariant with respect to Lorentz transformations" (Einstein, 1940 490; my emphasis).

"At first," as Einstein explained in private correspondence a few years later, in special relativity Swann "nothing is stated about the structural laws of nature other than the fact that they should be Lorentz-invariant" (Einstein to Swann, Jan. 24, 1942; EA 20-624). Indeed, "from the standpoint of our experience," the new kinematics could be considered as "better justified than any particular structural laws, e.g., Maxwell equations (Einstein to Swann, Jan. 24, 1942; EA 20-624)". The claim that the relativistic kinematics was 'better justified' than any particular law of nature is, in my view, the key to understand Einstein's comparison between thermodynamics and relativity theory. This hypothesis is confirmed by sparse but consistent textual evidence that can be gathered from several writings of this period. In Spring 1946, Einstein accepted (Einstein to Schilpp, May 29, 1946; EA 42-513) to write what he jokingly called his "autobiographical obituary" for the Schilpp-volume (Schilpp, 1949) in his honor. The manuscript (Einstein, 1946) was finished a few months later (Schilpp to Einstein, Feb. 8, 1947; EA 42-515). As for other issues, the text constitutes a fundamental document for the understanding of Einstein's relativity/thermodynamics analogy. Indeed, for the first time, Einstein claims that, in setting up special relativity, he had consciously chosen 
to follow the model of thermodynamics. Einstein's later recollections might be unreliable. What is interesting is the very fact that Einstein's use of the relativity theory/thermodynamics analogy switched from the 'context of justification' to the 'context of discovery.'

Einstein recalled that, already at the turn of the century, he had realized that Planck's radiation law "contradicts the mechanical and electrodynamic basis, upon which the derivation otherwise depends" (Einstein, 1946 17; tr. 1949a 45). In other terms, the thermodynamical equilibrium between matter and radiation could never come about if Newton and Maxwell equations held exactly. The energy of a mechanical system capable of oscillations (Planck's electromagnetic resonators representing the atomistic constituents of matter) probably did not vary continuously as in classical mechanics. Moreover, Einstein started to speculate that also the expression for the density of radiation energy implicitly presupposed by Maxwell equations needed to be challenged. Einstein claimed that he already knew in 1905, Maxwell's theory leads to incorrect fluctuations of radiation pressure of a freely moving mirror immersed in heat radiation as described by Planck's law (Einstein, 1946 17; tr. 1949a. 45; see above section 2.1.2). The 'electromechanical worldview' resting on Newtonian point mechanics and Maxwell's field equations started to stagger. As Einstein recollected, "[i]t was as if the ground had been pulled out from under one with no firm foundation to be seen anywhere, upon which one could have built" (Einstein, 1946, 17; tr. 1949a 45; see (Klein, 1980 Rynasiewicz and Renn, 2006). It was apparently in this context that Einstein decided to construct special relativity following the example represented by thermodynamics:

Reflections of this type made it clear to as long ago as 190o, shortly after Planck's trailblazing work, that neither mechanics nor electrodynamics ${ }^{33}$ could (except in limiting cases) claim exact validity. By and by I despaired of the possibility of discovering the true laws by means of constructive efforts [konstruktive Bem hüngen] based on known facts. The longer and the more despairingly I tried, the more I came to the conviction that only the discovery of a universal formal principle could lead us to the assured results. The example I saw before me was thermodynamics. The general principle was there given in the theorem: the laws of nature are such that it is impossible to construct a perpetuum mobile (of the first and second kind). How, then, could such a universal principle be found?.

[19][52]

This famous passage represents Einstein's direct attempt to explain the relativity/thermodynamics analogy. Einstein points out here more explicitly what he had only alluded to forty years earlier in the letter to Sommerfeld of January 1908 that we have quoted above (section 1.4.1): it was the failure of the electromechanical worldview that lead him to adopt an unconventional approach to the electrodynamics of moving bodies (section 1.3.2). Einstein could have attempted to find a solution to this problem by modifying classical mechanics and electrodynamics directly. Indeed, Einstein famously attempted to formulate an emission theory of light (Shankland, 1963). When all direct 'constructive efforts' failed, Einstein raised a different kind of question: "which is the law-making or constraining principle on which your constructive efforts should be based?" (Einstein to Waldinger, Mar. 12, 1946; EA, 27-358). This question represents the core of the principle strategy. Such 'constraining principles' can be found once we follow the "logical equivalent" of the strategy used in thermodynamics. "In both theories, it's about to derive deductively consequences from a general formal principle (in thermodynamics the postulate of the impossibility of a perpetuum mobile) where the formal principle is based on empirical basis)" (Einstein to Amiet, Dec. 17, 1947; EA, 25-335).

As Einstein explained in a letter to Robert Amiet written at the end of 1947, by setting up special relativity he could rely on "a plenty of experience, with the addition of Newton's mechanics, of the equivalence of all inertial frames," (Einstein to Amiet, Dec. 17, 1947; EA 25-335), an equivalence that could never violated despite all attempts. "A plenty of experiential knowledge" (Einstein to Amiet, Dec. 17, 1947; EA 25-335), with the addition of Lorentz-Maxwell theory, suggested "to consider the constancy of the vacuum speed of light as secure", thereby excluding emission theories of light. "The apparent incompatibility of these two principles [Grundsaetze]," Einstein further explained to Amiet, "forced to a critical consideration of the physical meaning of spatial and temporal coordinate in physics" (Einstein to Amiet, Dec. 17, 1947; EA 25-335). By dropping absolute simultaneity, Einstein could obtain the Lorentz transformations as the sought-for formal dependence between coordinates, which lets the speed of light invariant. This set of transformations is not arbitrary; once coordinates are interpreted as reading on rods and clocks, they can be true or false. There is nothing special "per se" in the variables $x, y, z, t$, if not for the fact that, in the fundamental laws of nature, dynamical are usually expressed as a singled-value function of the coordinates (Einstein, 1949c). Thus, the formal dependence between coordinates encoded in the Lorentz transformations enters into the formulation of all fundamental laws of nature imposing a strong constraint on the form that they can assume: "This is a restricting principle for natural laws, comparable to the restricting principle of the non-existence of the perpetuum mobile which underlies thermodynamics" (Einstein, 1946, 21; tr. 1949a. 57)

\footnotetext{
${ }^{33}$ The word 'thermodynamics' which appears in the manuscript and in the first English edition is a typo (see Abiko, 2003).
} 
The motivation of the relativity/thermodynamics analogy becomes then understandable. The two principles of thermodynamics can be derived independently of mechanics or of any particular theory, relying on wellestablished empirical facts, the impossibility of a perpetuum mobile of the first or second kind. Similarly, Einstein aimed to derive the Lorentz transformation independently of Maxwell electrodynamics, which "does not do justice to the energetic properties of radiation" (Einstein, 1949a 63). Einstein started from two empirically confirmed principles and asked how the laws of nature should look like if the two postulates have to hold. This "reversal of the trend" (Wigner, 1949) was the key point of Einstein strategy: "Maxwell equations imply the 'Lorentz group,' but the Lorentz group does not imply Maxwell equations” (Einstein, 1950, 14). Instead of being derived from Maxwell equations, in Einstein's approach, the Lorentz transformations are "defined independently of Maxwell equations," as the coordinate transformations, "which leave a particular value of the velocity-the velocity of light-invariant" (Einstein, 1950 14). Precisely because they are defined independently from Maxwell equations, such transformation equations become a "heuristic principle valid far beyond the range of the applicability or even validity of the equations themselves" (Einstein, 1950 14). The advantage of the theory consists then in the fact that Lorentz covariance "limits the possible natural laws in a definite manner" (Einstein, 1950 14), without having been derived by any of them. The drawback of this approach is that it tells "us what is possible but does not tell us what reality is" (Einstein in conversation with R.S. Shankland on February 4, 1950; in Shankland, 1963 49). Once relativistic kinematics has been stablished, constructive theories describing the dynamical behavior of matter and fields have to be set up-relativistic particle dynamics, electromagnetism, elasticity, must be developed. One should require that solutions to the dynamical equations of thoose theories exist that serve as a 'model' for the behavior of existing physical systems. ${ }^{34}$

Einstein explained more explicitly the rationale behind this indirect strategy in an often-quoted letter to Laue at the beginning of 1952. Laue, in his newly published 5th edition of his relativity textbook (Laue, 1952) had indicated Maxwell electrodynamics among the empirical confirmations of the special relativity. Einstein objected that this was misleading. Around 1905 (on the basis of his thought experiment of a Brownian motion of a mirror in a field of radiation), he was already convinced that Maxwell equations had to be modified in light of quantum phenomena. For this reason, special relativity "is based essentially only on the constant $c$, and not on the presupposition of the reality of the Maxwell field” (Einstein to Laue, Jan. 17, 1952; EA 16-168; my emphasis). Thus, in addition to the relativity postulate, Einstein-incapable to account for the structure of radiation-elevated only one isolated aspect of Maxwell's theory to the status of a second, more restrictive postulate. The new kinematic requirement is not dependent on the complete truth of Maxwell electrodynamics, but only on a small part of it which seemed robust to Einstein and was expressed by the light postulate. In the last fifty years, Einstein pointed out, nothing new has been discovered to explain the granular structure of radiation. Einstein hoped that this point of view could make Einstein quixotic search for a unified field theory more clear. "One cannot trust Maxwell equations," he wrote, "and, because of the general relativity principle, one has to rely of field and differential equations" (Einstein to Laue, Jan. 17, 1952; EA 16-168). If one relies on the principle of general covariance, the field concept becomes inevitable, and Einstein did not see any possible way other than a pure field theory, in spite of the challenge of deriving the atomistic and quantum structure of reality from it. Nevertheless, trusting the general principle of relativity was, according to Einstein, the only way when "one has come to despair of arriving at a deeper basis [Tieferlegung] of the theory by intuitive [anschaulich] constructive means" (Einstein to Laue, Jan. 17, 1952; EA, 16-168).

In spite of the usual rhetorics of the 'physics of desperation,' this was one of Einstein's most important and original methodological insights. In the same year, his first biographer Carl Seelig asked Einstein some comments about his 1919 London time article. In his reply, Einstein described his relativistic research as guided by the search for restrictive principles, "formal conditions which constraint [einschränken] the number of possible theories" (Einstein to Seelig, Jan. 1, 1952; EA 39-025). Einstein considered this an essential feature of his work. Einstein's major successes as a theorist had indeed been obtained by searching for progressively more restrictive 'requirements' to impose on the laws nature. special relativity ultimately introduced nothing but the restriction on the form that possible laws nature can assume. By providing a field-theoretical account of gravitation, general relativity, through its incorporation of the principle of general covariance, imposed a stronger restriction on any future physical theory that includes gravitation. If the principle of general covariance has to hold, reality should be represented by a continuous field, and the particle-like character should be deduced by the integration of a non-linear system of equations (Einstein to Mauritius Renninger, May 3, 1953; EA, 20-027). However, without the heuristic guide of a further restrictive principle, one does not have any clue as to what may be the mathematical structure of the 'total field.' One faces again the "dangerous obstacle in arbitrary choice (embarras de richesse)"

\footnotetext{
${ }^{34}$ Among such solutions there might be some that correspond to those rigid rods and ideal clocks that was initially introduced by hand. This shows that, sub specie aeterni, kinematics cannot be tested empirically separately from the rest of dynamical laws. Nevertheless, this does not change the fact that kinematics is more fundamental than the dynamics. If a dynamical laws contradict the relativistic kinematics, it is advisable to first attempt to change former, so that it adapt to the latter.
} 
(Einstein, 1949b 68o). In pursuit of a unified field theory, Einstein was forced to proceed in a "in a constructive way" (Einstein to Mauritius Renninger, May 3, 1953; EA 20-027), trying out new mathematical approaches and then discarding them when they did not produce the expected results. However, this procedure was usually less reliable: "I came to this opinion not only through the futility of many years of efforts but also through the experience of gravitational theory" (Einstein to De Broglie, Feb. 8, 1954; EA 8-311). The letter would be impossible to find, without a formal principle, the principle of general covariance, that restricts the range of possible generalizations of Poisson equations to essentially one single choice.

\section{Conclusion}

In 1953 Edmund Whittaker, a mathematician at the University of Edinburgh, finished the second volume of the new edition of his history of the aether theories (Whittaker, 1910). The volume (Whittaker, 1953) included a chapter entitled 'The Relativity Theory of Poincaré and Lorentz'. In the chapter, as one might guess from the title, Einstein's role in the relativity revolution was downplayed. Born, who was at that time working in Edinburgh and was a good friend of Whittaker, wrote to Einstein to warn him (Born to Einstein, Sep. 26, 1953; Born and Einstein, 1969 Doc. 102). Einstein did not show much interest (Einstein to Born, Oct. 2, 1953; Born and Einstein, 1969. Doc. 103). However, later, he offered a brief comment to his biographer Carl Seelig (1954) who had asked his opinion on the same matter (Seelig to Einstein, Feb. 17, 1955; EA. 39-070). The letter has become famous, and it offers a good summary of Einstein's stance towards the difference between his 'principle' approach and Lorentz's and Poincaré's 'constructive' one.

Einstein conceded that, around 1905, special relativity was, so to speak, in the air. "Lorentz had already recognized that the transformations named after him are essential for the analysis of Maxwell equations, and Poincaré deepened this insight still further" (Seelig to Einstein, Feb. 17, 1955; EA 39-o68; my emphasis). Einstein recalled that he was accustomed only to the older literature. However, most of all, he most of all emphasized what he thought were the two peculiarities of his approach: (1) "The new feature of it was the realization that the Lorentz transformation transcends its connection with Maxwell's equations and has to do with the nature of space and time in general", that is, with a reflection about the physical meaning of spacetime coordinates (Seelig to Einstein, Feb. 17, 1955; EA 39-o68). Once one interprets coordinates as measured with rods and clocks this new set of transformations laws becomes empirically testable independently from any particular dynamical law, including Maxwell equations. This latter aspect was "of particular importance," since, as we have seen, according to Einstein, "Maxwell's theory did not account for the microstructure of radiation and could, therefore, have no general validity" (Einstein to Seelig, Feb. 19, 1955; EA 39-070). But, of course, setting up new kinematics does bring about any further physical results. The next step is to recognize that "Lorentz invariance' is a general condition for any physical theory" (Einstein to Seelig, Feb. 19, 1955; EA 39-o68; my emphasis). Existing laws valid for low velocities that do not satisfy this condition have to be modified; through this adaptation, one obtains new relativistic laws. Some of the effects predicted by these modified theories could be tested empirically. As we have seen, this was precisely the strategy that leads to Einstein's successful derivation of the speed-dependent mass of the 'electron.' Both stages are essential: (1) The new kinematics based on the Lorentz coordinate transformations are not a byproduct of Maxwell equations and could be obtained without any reference to them (or to any other existing law of nature) from the two postulates given a physical meaning and compared to experience in terms of the behavior of rods and clocks (2) Precisely for this reason could assume the role of a constraint imposed to all possible laws of nature, in as much as coordinates enter into their formulation .

Einstein died in April 1955 a few months after this letter was drafted. On July 16, 1955, Born delivered a keynote lecture on relativity at the Bern conference on general relativity (Kervaire and Mercier, 1956) celebrating the 50 years of general relativity. On that occasion, Born mentioned the Seelig-Einstein correspondence in public for the first time. Born's remarks, it seems to me, grasp the essential point. According to Born, the letter shows that in Einstein's view, "the principle of relativity was more general and should be founded on considerations which would be still valid when Maxwell equations had to be discarded", that is replaced by a theory that would account for the discrete structure of radiation, such as "our present quantum electrodynamics" (Born, 1956b, 249). The difference between Einstein's approach and Lorentz-Poincaré approach lies precisely here, as Wolfgang Pauli pointed out just before his death in 1958 by commenting on the very same letter. According to Pauli, Lorentz or better Poincaré - who was the first to recognize the group property of the Lorentz transformations-"starts from the familiar equations of Maxwell and shows that they admit certain transformations" (Pauli, 1959 241; tr. 1994 118; my emphasis) . By contrast, Einstein sensed that Maxwell electrodynamics could not be generally correct. "He, therefore, formulated the invariance of the laws of nature with respect to Lorentz transformations as a general postulate which is more reliable than Maxwell equations" (Pauli, 1959, 241; my emphasis; tr. 1994, 119) . For this reason "[h]e established the postulate independently of these equations" (Pauli, 1959. 241; tr. 1994. 119; my emphasis) , from kinematic considerations "on the compatibility of the principle of relativity in translational motion with 
the principle of constancy of the velocity of light, assuming the relativity of simultaneity" (Pauli, 1959, 241; my emphasis, 1994 119).

These few remarks reveal the rationale behind Einstein's characterization of special relativity as a principle theory. As Cornelius Lanczos-Einstein's former assistant and long-life correspondent-made a similar point in a celebratory article written after Einstein's death. The Lorentz transformation, he wrote, "occurred in consequence of certain mathematical properties of Maxwell equations and were investigated by Lorentz and Poincaré " (Lanczos, 1955 1202). However, "it was Einstein, who discovered the proper interpretation of the Lorentz transformations as relations between coordinates". As a consequence, "all equations of physics had to be revised, in order to bring them in harmony with the relativity principle" (Lanczos, 1955 1202; my emphasis). In this way, as Lanczos wrote some years later, Einstein transformed physics into a two-floor building. Previously physics remained on the first floor, attempting "to find some mathematical law which will fit the experiments", Einstein erected a "a new second floor", "in which the mathematical law is no longer accepted as a more or less accidental description of natural events", but as a consequence "of some sweeping philosophical principles" (Lanczos, 1959 51). The success of Einstein's work derived from the fact that he "never dealt with specific equations but with all-comprehensive principles from which profound consequences could be deduced" (Lanczos, 1965).

The characterization of a second-order theory seems indeed seems how Einstein's theory was percieved by early relativists. As Valentine Bargmann, another Einstein's former assistant,put it, in the early years, the goal was "to extend relativity to various parts of physics by adjusting already existing theories to the relativistic postulates" (Bargmann, 1960 190; my emphasis). For small velocities, the theories could be taken for granted, and "although their relativistic generalization required ingenuity and penetrating analysis", "no radical change" was needed. With the progress of atomic physics and especially of quantum theory, the role of special relativity revealed in stronger heuristic power (Bargmann, 1960 191). "The problem was no longer to 'translate' a previously established theory into a relativistic form" (Bargmann, 1960 191), but increasingly "the theories were relativistic from the start, and relativistic considerations were crucial for the choice of their basic postulates" (Bargmann, 1960 191). Dirac's theory of the electron, e.g., "was not obtained from an established non-relativistic theory, but derived from basis quantum theoretical and relativistic postulates" (Bargmann, 1960 191). The relativistic energy-momentum relation uniquely determined the form of the Dirac Hamiltonian leading to the four components of the wave function (together with their curious transformation properties) and to the electronic spin (Bargmann, 1960 191).

As it is well-known, the current debate about the foundation of spacetime-theories has taken the form of the opposition between a geometrical and a dynamical approach, that is, a difference between two kinds of 'explanations.' In both cases, relativity theory is ultimately treated as a constructive theory: a constructive theory of the material structure of rods and clocks (Brown) or a constructive theory of the geometrical structure of spacetime (Janssen). As it has been rightly pointed out, these are ultimately only two sides of the same coin (Acuña, 2014). In this sense, the geometrical/dynamical opposition does not capture the critical difference between the Einstein/Minkowski and Lorentz/Poincaré approach. The textual evidence that we have presented seems to show that the real issue at stake can be better framed by resorting to Lange's (2007) opposition between 'byproducts' and 'constraints.' For Lorentz and Poincaré, invariance with respect to transformations was a byproduct of certain laws governing field and matter, it was a formal feature that they happen to posses. Einstein derived the Lorentz transformations independently of any dynamical laws, from generalizable facts summarized in the two postulates and elevate the invariance respect to such transformations to a constraint that all laws of nature must posses. What both the geometrical and dynamical approach fails to grasp is this peculiar modal status of the requirement of Lorentz invariance, which is a normative and not a factual claim, either about the structure of matter or of spacetime. It is the latter that transforms Lorentz invariance from a 'analytic' principle satisfied by the existing laws, into a 'synthetic' principle from which one can extract new laws starting from the existing ones.

In my view, Lange's parlance encapsulates the two senses in which characterization of relativity theory as 'theory of principles' appears in Einstein's writings. On the one hand, the expression indicates (a) a class of existing theories: principle theories unlike constructive theories, do not directly say anything about the laws governing specific physical system, rather they put constraints on them (b) a strategy for finding new theories: instead of searching directly for the laws of nature, it is often more appropriate to first search for constraints the limit the number of possible candidates. Thus, the relationship between constructive and principle theories is not that between explanation and what is to be explained, as today scholars usually claim, but, so to speak, but between a norm and the cases covered by it. Constructive theories are instances of theories that are allowed by the constraints established by principle theories. Thus, principle theories are more general and fundamental than constructive theories. Nevertheless, only constructive theories are physical theories in the proper sense of the expression. The goal of physics is not merely to limit the number of possible constructive theories but to single out the only possibility that, as Einstein might have put it, 'the Lord has chosen.' However, Einstein realized that the direct search for constructive theories is often not the right heuristic strategy, and one should first find a way to limit the number of possible candidates. In this sense, Einstein was indeed a Prinzipienfuchser, a principle 
pincher who holds on to few fundamental principles that limit the number of theoretical possibilities rather than indulging in the mathematical virtuosity necessary to explore these possibilities one after another.

\section{Abbreviations}

ASWB Arnold Sommerfeld (200o-2004). Wissenschaftlicher Briefwechsel. Ed. by Michael Eckert. 2 vols. Berlin: Verlag für Geschichte der Naturwissenschaften und der Technik, 2000-2004

CPAE Albert Einstein (1987-). The Collected Papers of Albert Einstein. Ed. by John Stachel et al. 15 vols. Princeton: Princeton University Press, 1987-

DMA Deutsches Museum, Archiv. München.

EA The Albert Einstein Archives at the Hebrew University of Jerusalem.

ESC Ehrenfest Scientific Correspondence. As catalogued in Wheaton, Bruce R., Catalogue of the Paul Ehrenfest Archive at the Museum Boerhaave Leiden. Leiden: Museum Boerhaave, 1977.

MGA Hermann Minkowski (1911). Gesammelte Abhandlungen. Ed. by David Hilbert, Andreas Speiser, and Hermann Weyl. Leipzig: Teubner, 1911.

SCHAL Hendrik Antoon Lorentz (2008-). The Scientific Correspondence of H.A. Lorenzt. Ed. by A. J. Kox. New York: Springer, 20o8-. I thank A.J. Kox for giving me access to the unpublished vol. 2 .

\section{References}

Abiko, Seiya (2003). “On Einstein's Distrust of the Electromagnetic Theory. The Origin of the Light-velocity Postulate.” Historical Studies in the Physical and Biological Sciences 33, 193-215.

Abraham, Max (1902a). “Dynamik des Electrons.” Vorgelegt von Herrn W. Voigt in der Sitzung vom 11. Januar 1902. Göttinger Nachrichten, $20-41$.

- (1902b). "Geometrische Grundbegriffe." In: vol. 4, part 3. 1902, 3-47.

- (1902c). "Prinzipien der Dynamik des Elektrons." Annalen der Physik 315, 105-179.

- (1903). "Prinzipien der Dynamik des Elektrons." Physikalische Zeitschrift 4, 57-63.

- (1904a). "Die Grundhypothesen der Elektronentheorie." Physikalische Zeitschrift 5, 576-579.

- (1904b). "Zur Theorie der Strahlung und des Strahlungsdruckes." Annalen der Physik. 4th ser. 13, 236-287.

- (1905a). "Die Bewegungsgleichungen eines Massen teilchens in der Relativtheorie." Physikalische Zeitschrift 11, 527-531.

- (1905b). Theorie der Elektrizität. Vol. 2: Elektromagnetische Theorie der Strahlung. Leipzig: Teubner, 1905.

- (1908). Theorie der Elektrizität. 2nd ed. Vol. 2: Elektromagnetische Theorie der Strahlung. Leipzig: Teubner, 1908.

- (1909a). "Zur Elektrodynamik bewegter Körper." Rendiconti del Circolo Matematico di Palermo 28, 1-28.

- (1909b). "Zur elektromagnetischen Mechanik.” Physikalische Zeitschrift 10, 737-741.

- (1910a). "Die Bewegungsgleichungen eines Massenteilchens in der Relativtheorie.” Physikalische Zeitschrift 11, 527-31.

- (1910b). "Sull'elettrodinamica di Minkowski." Rendiconti del Circolo Matematico di Palermo 30, 33-46.

- (1912a). "Nochmals Relativität und Gravitation. Bemerkungen zu A. Einsteins Erwiderung." Annalen der Physik. 4th ser. 344, $444-448$.

- (1912b). "Una nuova teoria della gravitazione." Conferenza tenuta alla socità per il progresso delle scienze a Genova il 19 Ottobre 1912 . Il Nuovo Cimento 4, 459-481.

- (1912c). "Zur Theorie der Gravitation." Physikalische Zeitschrift 13, 1-4.

Acuña, Pablo (2014). "On the Empirical Equivalence between Special Relativity and Lorentz's Ether Theory." Studies in History and Philosophy of Science. Part B: Studies in History and Philosophy of Modern Physics 46, 283-302.

Balashov, Yuri and Michel Janssen (2003). "Presentism and Relativity.” The British fournal for the Philosophy of Science 54, 327-346.

Bargmann, Valentine (1960). "Relativity.” In: 1960, 187-198.

Barkan, Diana Kormos (1999). Walther Nernst and the Transition to Modern Physical Science. Cambridge: Cambridge University Press, 1999.

Beller, Mara (1999). Quantum Dialogue. The Making of a Revolution. Chicago: University of Chicago Press, 1999.

Bestelmeyer, Adolf (1907). "Spezifische Ladung und Geschwindigkeit der durch Röntgenstrahlen erzeugten Kathodenstrahlen.” Annalen der Physik. 4 th ser. $327,429-447$.

Bevilacqua, Fabio (1993). "Helmholtz's Ueber die Erhaltung der Kraft. The Emergence of a Theoretical Physicist.” In: Hermann Von Helmholtz and the Foundations of Nineteenth-century Science. Ed. by David Cahan. Berkeley: University of California Press, 1993, 291-333.

Boltzmann, Ludwig (1896-1898). Vorlesungen über Gastheorie. 2 vols. Leipzig: Barth, 1896-1898. Lectures on Gas Theory. Trans. by Stephen G. Brush. Berkeley: University of California Press, 1964.

Bordoni, Stefano (2017). When Historiography Met Epistemology. Sophisticated Histories and Philosophies of Science in French-speaking Countries in the Second Half of the Nineteenth Century. Leiden/Boston: Brill, 2017.

Born, Max (1909a). "Die Theorie des starren Elektrons in der Kinematik des Relativitätsprinzips.” Annalen der Physik. 4th ser. $29,1-56$.

- (1909b). "Die träge Masse und das Relativitätsprinzip." Annalen der Physik. $4^{\text {th }}$ ser. 28, 571-584.

- (1909c). "Über die Dynamik des Elektrons in der Kinematik des Relativitätsprinzips." Physikalische Zeitschrift 10, 814-817.

- (1910a). "Über die Definition des starren Körpers in der Kinematik des Relativitätsprinzips." Physikalische Zeitschrift 11, $233-234$.

- (1910b). "Zur Kinematik des starren Körpers im System des Relativitätsprinzips.” Nachrichten von der Königlichen Gesellschaft der Wissenschaften zu Göttingen, Mathematisch-physikalische Klasse, 161-179.

- (1911). "Elastizitätstheorie und Relativitätstheorie." Physikalische Zeitschrift 12, 569-575

- (1915). Dynamik der Kristallgitter. Leipzig: Teubner, 1915.

- (1956a). Experiment and Theory in Physics. 2nd ed. New York: Dover, 1956.

- (1956b). "Physics and Relativity." In: Fünfzig Fahre Relativitätstheorie - Cinquantenaire de la théorie de la relativité - Jubilee of relativity theory. Ed. by Michel A. Kervaire and André Mercier. Basel: Birkhäuser, 1956, 244-260.

Born, Max and Albert Einstein (1969). Briefwechsel, 1916-1955. Ed. by Hedwig Born and Max Born. München: Nymphenburger Verlagshandlung, 1969. Max Born, Hedwig Born, and Albert Einstein. The Born-Einstein Letters. Correspondence between Albert Einstein and Max and Hedwig Born from 1916 to 1955 with commentaries by Max Born. London/Basingstoke: MacMillan, 1968-1971.

Bracco, Christian and Jean-Pierre Provost (2009). "De l'électromagnétisme à la mécanique. Le rôle de l'action dans le Mémoire de Poincaré de 1905." Revue d'histoire des sciences 62, 457.

Brown, Harvey R. (2005). Physical Relativity. Space-time Structure from a Dynamical Perspective. Oxford: Clarendon, 2005. 
Brown, Harvey R. and Oliver Pooley (2006). “Minkowski Space-Time. A Glorious Non-entity.” In: The Ontology of Spacetime. Ed. by Dennis Dieks. Vol. 1. 2 vols. Amsterdam: Elsevier, 2006, 67-89.

Bub, Jeffrey (200o). "Quantum Mechanics As a Principle Theory." Studies in History and Philosophy of Science. Part B: Studies in History and Philosophy of Modern Physics 31, 75-94.

Bucherer, Alfred Heinrich (1904). Mathematische Einführung in die Elektronentheorie. Leipzig: Teubner, 1904.

- (1905). "Das deformierte Elektron und die Theorie des Elektromagnetismus." Physikalische Zeitschrift 6, 833-834.

- (1908). "Messungen an Becquerelstrahlen. Die experimentelle Bestätigung der Lorentz-Einsteinschen Theorie.” Physikalische Zeitschrift 9, $755^{-762 .}$

- (1909). "Die experimentelle Bestätigung des Relativitätsprinzips.” Annalen der Physik. 4th ser. 28, 513-536.

Bucherer, Alfred Heinrich et al. (1908). "Discussion following Bucherer, 'Messungen an Becquerelstrahlen' [Bucherer, 1908]. Die experimentelle Bestätigung der Lorentz-Einsteinschen Theorie." Physikalische Zeitschrift 9, 755-762.

Clifton, Rob, Jeffrey Bub, and Hans Halvorson (2003). "Characterizing Quantum Theory in Terms of Information-Theoretic Constraints." Foundations of Physics 33, 1561-1591.

Cohn, Emil (1904a). "Zur Elektrodynamik bewegter Systeme." Sitzungsberichte der Königlich Preußischen Akademie der Wissenschaften, Halbband 2, 1294-1303.

- (1904b). "Zur Elektrodynamik bewegter Systeme.” II. Sitzungsberichte der Königlich Preußischen Akademie der Wissenschaften, Halbband 2, $1404-1416$.

Corry, Leo (1998). "The Influence of David Hilbert and Hermann Minkowski on Einstein's Views Over the Interrelation between Physics and Mathematics." Endeavour 22, 95-97.

- (2004). David Hilbert and the Axiomatization of Physics (1898-1918). From Grundlagen der Geometrie to Grundlagen der Physik. Dordrecht: Kluwer, 2004

- (2010). "Hermann Minkowski, Relativity and the Axiomatic." In: Minkowski Spacetime. A Hundred Years Later. Ed. by Vesselin Petkov. Dordrecht et al.: Springer, 2010, 3-41.

Cushing, James T. (1981). "Electromagnetic Mass, Relativity, and the Kaufmann Experiments." American fournal of Physics 49, $1133-1149$.

- (200o). Philosophical Concepts in Physics. The Historical Relation between Philosophy and Scientific Theories. Cambridge: Cambridge University Press, 2000.

Darrigol, Olivier (1995a). "Emil Cohn's Electrodynamics of Moving Bodies." American fournal of Physics 63, 908-915.

- (1995b). "Henri Poincaré's Criticism of fin De Siécle Electrodynamics." Studies in History and Philosophy of Science. Part B: Studies in History and Philosophy of Modern Physics 26, 1-44.

- (2018). Atoms, Mechanics, and Probability. Ludwig Boltzmann's Statistico-Mechanical Writings - an Exegesis. Oxford: Oxford University Press, 2018.

Deltete, Robert (1999). "Helm and Boltzmann." Synthese. Energetics at the Lübeck Naturforscherversammlung 119, 45.

Deltete, Robert J. (2012). "Planck, Ostwald, and the Second Law of Thermodynamics." HOPOS: The fournal of the International Society for the History of Philosophy of Science 2, 121-146.

Dieks, Dennis (2009). "Understanding in physics: Bottom-up versus top-down." In: Scientific Understanding: Philosophical Perspectives. Ed. by Henk W. de Regt, Sabina Leonelli, and Kai Eigne. Pittsburgh: University of Pittsburgh Press, 2009.

Dongen, Jeroen van (2010). Einstein's Unification. Cambridge/New York: Cambridge University Press, 2010.

Dorato, Mauro (2011). "Relativity Theory between Structural and Dynamical Explanations." International Studies in the Philosophy of Science 21, 95-102.

Ed. Note. "Einstein And Laub on the Electrodynamics of Moving Media." CPAE Vol. 2, 504-507.

Eddington, Arthur Stanley (1921). "A Generalization of Weyl's Theory of the Electromagnetic and Gravitation fields." Proceedings of the Royal Society London 99, 104-121.

Ehrenfest, Paul (1906a). "Über die physikalischen Voraussetzungen der Planck'schen Theorie der irreversiblen Strahlungsvorgänge.” Sitzungsberichte der Kaiserliche Akademie der Wissenschaften in Wien, Mathematisch-physikalische Klasse, 1301-1314.

- (1906b). "Zur Planckschen Strahlungstheorie." Physikalische Zeitschrift 7, 2-6.

- (1906c). "Zur Stabilitätsfrage bei den Bucherer-Langevin Elektronen.” Physikalische Zeitschrift 7, 302-303.

- (1907). "Die Translation deformierbarer Elektronen und der Flächensatz." Annalen der Physik. 4th ser. 23, 204-205.

- (1909). "Gleichförmige Rotation starrer Körper und Relativitätstheorie.” Physikalische Zeitschrift 10, 918.

- (1910). "Zu Herrn v. Ignatowskys Behandlung der Bornschen Starrheitsdefinition.” Physikalische Zeitschrift 11, $1127-1129$.

- (1911). "Zu Herrn v. Ignatowskys Behandlung der Bornschen Starrheitsdefinition II." Physikalische Zeitschrift 12, 412-413.

Einstein, Albert (1905a). Eine neue Bestimmung der Moleküldimensionen: Inaugural-Dissertation zur Erlangung der philosophischen Doktorwürde der hohen Philosophischen Fakultät (Mathematisch-Naturwissenschaftliche Sektion) der Universität Zürich. Bern: Buchdruckerei K. J. Wyss, 1905. Repr. in CPAE Vol. 2, Doc. 16.

- (1905b). "Ist die Trägheit eines Körpers von seinem Energieinhalt abhängig?" Annalen der Physik. 4th ser. 18, 639-641. Repr. in CPAE Vol. 2, Doc. 24 .

- (1905c). "Über die von der molekularkinetischen Theorie der Wärme geforderte Bewegung von in ruhenden Flüssigkeiten suspendierten Teilchen." Annalen der Physik. 4th ser. 17, 549-56o. Repr. in CPAE Vol. 2, Doc. 16

- (1905d). "Über einen die Erzeugung und Verwandlung des Lichtes betreffenden heuristischen Gesichtspunkt." Annalen der Physik. 4 th ser. 17, 132-148. Repr. in CPAE Vol. 2, Doc. 14.

- (1905e). "Zur Elektrodynamik bewegter Körper." Annalen der Physik. 4th ser. 17, 891-921. Repr. in CPAE. Vol. 2, Doc. 23.

- (1906a). "Das Prinzip von der Erhaltung der Schwerpunktsbewegung und die Trägheit der Energie.” Annalen der Physik. 4th ser. 20, $627-633$.

- (1906b). "Eine Methode zur Bestimmung des Verhältnisses der transversalen und longitudinalen Masse des Elektrons." Annalen der Physik. 4 th ser. $21,583-586$.

- (1906c). "Eine neue Bestimmung der Moleküldimensionen." Annalen der Physik. 4th ser. 19, 289-305

- (1906d). "Theorie der Lichterzeugung und Lichtabsorption." Annalen der Physik. 4th ser. 20, 199-206. Repr. in CPAE. Vol. 2, Doc. 34.

- (1907a). "Bemerkungen zu der Notiz von Hrn. Paul Ehrenfest: 'Die Translation deformierbarer Elektronen und der Flächensatz'." Annalen

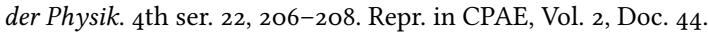

- (1907b). "Plancksche Theorie der Strahlung und die Theorie der Spezifischen Wärme." Annalen der Physik. 4th ser. 22, 180-19o, 8oo. Repr. in CPAE Vol. 2, Doc. 38.

- (1907c). "Über die Gültigkeitsgrenze des Satzes vom thermodynamischen Gleichgewicht und über die Möglichkeit einer neuen Bestimmung

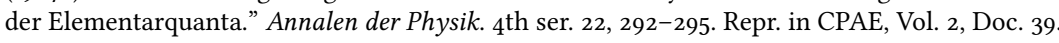


Einstein, Albert (1907d). "Über die vom Relativitätsprinzip geforderte Trägheit der Energie.” Annalen der Physik. 4th ser. 23, 371-384. Repr. in CPAE Vol. 2, Doc. 45 .

- (1908). "Relativitätsprinzip und die aus demselben gezogenen Folgerungen.” Jahrbuch der Radioaktivität und Elektronik 4, 411-462. Repr. in CPAE Vol. 2, Doc. 47.

- (1909a). "Über die Entwicklung unserer Anschauungen über das Wesen und die Konstitution der Strahlung." Vorgetragen in der Sitzung der physikalischen Abteilung der 81. Versammlung Deutscher Naturforscher und Ärzte zu Salzburg am 21. September 1909. Verhandlungen der Deutschen Physikalischen Gesellschaft 7, 482-50o. Repr. in CPAE Vol. 2, Doc. 6o. Also pub. in: Physikalische Zeitschrift 10, 1909, 817-825.

- (1909b). "Über die Entwicklung unserer Anschauungen über das Wesen und die Konstitution der Strahlung." Vorgetragen in der Sitzung der physikalischen Abteilung der 81. Versammlung Deutscher Naturforscher und Ärzte zu Salzburg am 21. September 1909. Physikalische Zeitschrift 10, 817-825.

- (1909c). "Zum gegenwärtigen Stand des Strahlungsproblems." Physikalische Zeitschrift 10, 185-193. Repr. in CPAE Vol. 2, Doc. 56.

- (1910a). "Le principe de relativité et ses conséquences dans la physique moderne.” Trans. by Edouard Guillaume. Archives des sciences physiques et naturelles 29, 5-28, 125-144. Repr. in CPAE Vol. 3, Doc. 2.

- (1910b). Response to Manuscript of (Planck, 1910b). 1910. pub. in CPAE Vol. 3, Doc. 3.

- (1910c). "Sur la théorie des quantités lumineuses et la question de la localisation de l'énergie électromagnetique." Archives des sciences physiques et naturelle 29, 525-528. Repr. in CPAE Vol. 3, Doc. 5

- (1910d). "Theorie der Opaleszenz von homogenen Flüssigkeiten und Flüssigkeitsgemischen in der Nähe des kritischen Zustandes.” Annalen der Physik. $4^{\text {th ser. } 14,1276-1298 .}$

- (1910e). "Über das Boltzmannsche Prinzip und einige unmittelbar aus demselben fliessende Folgerungen.” 1910. Pub. in CPAE Vol. 3, Doc. 10a (Vol. 13).

- (1911a). "[Comment on a Fundamental Difficulty in Theoretical Physics]." 1911. Pub. in CPAE Vol. 3, Doc. 16.

- (1911b). "[Statement on the Light Quantum Hypothesis]." Sitzungsberichte der Naturforschenden Gesellschaft in Zürich, XVI. Pub. in CPAE Vol. 3, Doc. 20

- (1911c). "Die Relativitäts-Theorie.” Naturforschende Gesellschaft in Zürich, Vierteljahresschrift 56, 1-14. Repr. in CPAE. Vol. 3, Doc. 17.

- (1911d). “Diskussion von 'Die Relativitäts-Theorie' [Einstein, 1911c].” Naturforschende Gesellschaft in Zürich, Vierteljahresschrift 56, II-IX. Repr. in CPAE Vol. 3, Doc. 18.

- (1911e). "Zum Ehrenfestschen Paradoxon. Bemerkung zu V. Varićaks Aufsatz." Physikalische Zeitschrift 12, 509-510.

- (1912-1914). "Einstein's Manuscript on the Special Theory of Relativity." 1912-1914. Pub. in CPAE. Vol. 4, Doc. 1.

- (1912a). "Lichtgeschwindigkeit und Statik des Gravitationsfeldes." Annalen der Physik. 4th ser. 38, 355-369. Repr. in CPAE Vol. 4, Doc. 3.

- (1912b). "Relativität und Gravitation. Erwiderung auf eine Bemerkung von M. Abraham." Annalen der Physik. 4th ser. 38, 1059-1064.

- (1912c). "Thermodynamische Begründung des photochemischen Äquivalentgesetzes." Annalen der Physik. 4th ser. 37, 832-838. Repr. in CPAE. Vol. 4, Doc. 2.

- (1913a). "Gravitationstheorie." Schweizerische Naturforschende Gesellschaft, Verhandlungen, 96, 137-138. Repr. in CPAE Vol. 15, Doc. 4.

- (1913b). "Max Planck als Forscher." Die Naturwissenschaften 1, 1077-1079. Repr. in CPAE. Vol. 4, Doc. 23.

- (1913c). "Zum gegenwärtigen Stande des Gravitationsproblems.” Physikalische Zeitschrift 14, 1249-1266, Diskussion 1262-1266. Repr. in CPAE Vol. 4, Doc. 17 .

- (1914a). “Antrittsrede des Hrn. Einstein.” Sitzungsberichte der Preußischen Akademie der Wissenschaften, Halbband 1, 739-742. Repr. in CPAE Vol. 3, Doc. 18.

- (1914b). "Die formale Grundlage der allgemeinen Relativitätstheorie." Sitzungsberichte der Preußischen Akademie der Wissenschaften, Halbband 2, 1030-1085. Repr. in CPAE Vol. 6, Doc. 9.

- (1914c). "Prinzipielles zur verallgemeinerten Relativitätstheorie." Physikalische Zeitschrift 15, 176-18o. Repr. in CPAE. Vol. 4, Doc. 25.

- (1914d). "Vom Relativitäts-Prinzip." Vossische Zeitung. 1914. Repr. in CPAE Vol. 6, Doc. 1.

- (1914e). "Zum gegenwärtigen Stande des Problems der spezifischen Wärme." In: Die Theorie der Strahlung und der Quanten. Verhandlungen auf einer von E. Solvay einberufenen Zusammenkunft (30. Oktober bis 3. November 1911), mit einem Anhange über die Entwicklung der Quantentheorie vom Herbst 1911 bis Sommer 1913. Ed. by Arnold Eucken. Halle: Knapp, 1914, 330-352. Repr. in CPAE Vol. 3, Doc. 26.

- (1914f). "Zum Relativitäts-Problem." Scientia 15, 337-348. Repr. in CPAE Vol. 4, Doc. 31.

- (1915a). Die Relativitätstheorie. In: Die Kultur der Gegenwart. Ihre Entwicklung und ihre Ziele. Ed. by Paul Hinneberg. Leipzig: Teubner, 1915, Part 3, Sec. 1, Vol. 3: Physik. Ed. by Emil Warburg, 703-713. Repr. in CPAE Vol. 4, Doc. 21.

- (1915b). "Erklärung der Perihelbewegung des Merkur aus der allgemeinen Relativitätstheorie." Sitzungsberichte der Preußischen Akademie der Wissenschaften, Halbband 2, 831-839. Repr. in CPAE. Vol. 6, Doc. 24

- (1915c). "Feldgleichungen der Gravitation." Sitzungsberichte der Preußischen Akademie der Wissenschaften, Halbband 2, 844-847. Repr. in CPAE Vol. 5, Doc. 25 .

- (1915d). "Grundgedanken der allgemeinen Relativitätstheorie und Anwendung dieser Theorie in der Astronomie." Sitzungsberichte der Preußischen Akademie der Wissenschaften, Halbband 1, 315.

- (1915e). "Zur allgemeinen Relativitätstheorie." Sitzungsberichte der Preußischen Akademie der Wissenschaften, Halbband 2, 778-786, 799-801. Repr. in CPAE. Vol. 6, Doc. 21 and 22.

- (1915f). "Zur allgemeinen Relativitätstheorie." Sitzungsberichte der Preußischen Akademie der Wissenschaften, Halbband 2, 778-786. Repr. in CPAE Vol. 6, Doc. 21.

- (1915g). “Zur allgemeinen Relativitätstheorie (Nachtrag).” Sitzungsberichte der Preußischen Akademie der Wissenschaften, Halbband 2, 799-801. Repr. in CPAE Vol. 6, Doc. 22.

- (1916a). "Die Grundlage der allgemeinen Relativitätstheorie." Annalen der Physik. 4th ser. 49, 769-822. Also issued as a separatum, Leipzig: Barth. Repr. in CPAE Vol. 6, Doc. 3o.

- (1916b). "Hamiltonsches Prinzip und allgemeine Relativitätstheorie." Sitzungsberichte der Preußischen Akademie der Wissenschaften, 1111-1116. Repr. in CPAE Vol. 6, Doc. 41.

- (1917a). "Die hauptsächlichen Gedanken der Relativitätstheorie." CPAE Vol. 6[7], Doc. 44a. 1917.

- (1917b). Über die spezielle und die allgemeine Relativitätstheorie (gemeinverständlich). Braunschweig: Vieweg, 1917. Repr. in CPAE Vol. 5, Doc. 42 .

- (1918a). "Dialog über Einwände gegen die Relativitätstheorie." Die Naturwissenschaften 6, 697-702. Repr. in CPAE Vol. 7, Doc. 13.

- (1918b). Motive des Forschens. In: Zu Max Plancks sechzigstem Geburtstag. Ansprachen, gehalten am 26. April 1918 in der Deutschen Physikalischen Gesellschaft von E. Warburg, M. v. Laue, A. Sommerfeld und A. Einstein. Ed. by Emil Warburg et al. Karlsruhe: C. F. Müllersche Hofbuchhandlung, 1918, 29-32. Repr. in CPAE Vol. 7, Doc. 7.

- (1919a). "Lecture Notes for Course on General Relativity at the University of Berlin." Summer Semester. 1919. Pub. in CPAE. Vol. 7, Doc. 19. 
Einstein, Albert (1919b). “Spielen Gravitationsfelder im Aufbau der materiellen Elementarteilchen eine wesentliche Rolle?” Sitzungsberichte der Preußischen Akademie der Wissenschaften, Physikalisch-mathematische Klasse, 349-356. Repr. in CPAE Vol. 7, Doc. 17.

- (1919c). "Was ist Relativitäts-Theorie." 1919. The original German manuscript is transcribed in CPAE Vol. 7, Doc. 25.

- (1919d). "What is the Theory of Relativity?" The Times. 1919. Repr. in CPAE Vol. 7, Doc. 25

- (1920a). “Antwort auf vorstehende Betrachtung [Reichenbächer, 1920].” Die Naturwissenschaften 8, 1010-1011. Repr. in CPAE Vol. 7, Doc. 49.

- (1920b). "Grundgedanken und Methoden der Relativitätstheorie, in ihrer Entwicklung dargestellt." 1920. Unpublished 35-page draft of an article for Nature. Published in CPAE Vol. 7, Doc. 31.

- (1921a). “King's College Lecture." Before June 13. 1921. Pub. in CPAE Vol. 7, Doc. 58

- (1921b). "Über eine naheliegende Ergänzung des Fundamentes der allgemeinen Relativitätstheorie.” Sitzungsberichte der Preußischen Akademie der Wissenschaften, Halbband 1, 261-264. Repr. in CPAE Vol. 7, Doc. 54.

- (1923a). "The Theory of the Affine field." Nature 2812, 448-449. Repr. in CPAE Vol. 14, Doc. 123

- (1923b). "Zur affinen Feldtheorie.” Sitzungsberichte der Preußischen Akademie der Wissenschaften, Physikalisch-mathematische Klasse, 137-140. Repr. in CPAE Vol. 14, Doc. 52.

- (1923c). "Zur allgemeinen Relativitätstheorie." Sitzungsberichte der Preußischen Akademie der Wissenschaften, Physikalisch-mathematische Klasse, 32-38, 76-77. Repr. in CPAE Vol. 13, Doc. 425.

- (1925a). "Einheitliche Feldtheorie von Gravitation und Elektrizität." Sitzungsberichte der Preußischen Akademie der Wissenschaften, Physikalisch-mathematische Klasse, 414-419. Repr. in CPAE Vol. 15, Doc. 17.

- (1925b). "La fçsica y la esencia de las cosas." La Prensa. 1925. Repr. in CPAE. Vol. 14, Doc. 481.

- (1927a). "Über die formale Beziehung des Riemannschen Krümmungstensors zu den Feldgleichungen der Gravitation.” Mathematische Annalen 97, 99-103.

- (1927b). "Zu Kaluzas Theorie des Zusammenhanges von Gravitation und Elektrizität.” Erste und zweite Mitteilung. Sitzungsberichte der Preußischen Akademie der Wissenschaften, Physikalisch-mathematische Klasse, 23-25, 26-30.

- (1927c). "Zu Kaluzas Theorie des Zusammenhanges von Gravitation und Elektrizität." Zweite Mitteilung. Sitzungsberichte der Preußischen Akademie der Wissenschaften, Physikalisch-mathematische Klasse, 26-30.

- (1928). “A propos de 'La Déduction Relativiste' de M. Émile Meyerson [Meyerson, 1925." Revue philosophique de la France et de l'étranger $105,161-166$.

- (1933). Einiges über die Entstehung der allgemeinen Relativitätstheorie. EA 5-3.

- (1935). "Elementary Derivation of the Equivalence of Mass and Energy." Bulletin of the American Mathematical Society 41, 223-230.

- (1940). "Considerations concerning the Fundaments of Theoretical Physics." Science 91, 487-492.

- (1946). "[Autobiographisches]." EA 2-21; pub. with an english tr. as Einstein, $1949 \mathrm{a} 1946$.

- (1949a). “Autobiographical Notes.” In: Albert Einstein, Philosopher-Scientist. Philosopher-Scientist Ed. by Paul Arthur Schilpp. Evanston: The Library of Living Philosophers, 1949, 2-94.

- (1949b). "Remarks Concerning the Essays Brought Together in This Co-operative Volume." In: Albert Einstein. Philosopher-Scientist Ed. by Paul Arthur Schilpp. Evanston, Ill.: The Library of Living Philosophers, 1949, 665-688.

- (1949c). Unpub. Reply to A. P. Uschenko. EA 206o.

- (1950). "On the Generalized Theory of Gravitation." Scientific American 182, 13-17.

Einstein, Albert et al. (1912). "Discussion du rapport de M. Einstein.” In: La theorie du rayonnement et les quanta. Rapports et discussions de Ia reunion tenue aBruxelles, du 30 octobre au 3 novembre 1911, sous les auspices de M. E. Solvay. Ed. by Paul Langevin and Maurice de Broglie. Paris: Gauthier-Villars, 1912, 436-450. Later published in German as Einstein et al., 1914

- (1914). “Discussion' Following the Lecture Version of 'Zum gegenwärtigen Stande des Problems der spezifischen Wärme' (Einstein, 1914e." In: Die Theorie der Strahlung und der Quanten. Verhandlungen auf einer von E. Solvay einberufenen Zusammenkunft (3o. Oktober bis 3. November 1911), mit einem Anhange über die Entwicklung der Quantentheorie vom Herbst 1911 bis Sommer 1913. Ed. by Arnold Eucken. Vol. 3. Halle: Knapp, 1914, 353-364. Printed version of the discussion held on November 3, 1911 following presentation of Einstein's paper at the first Solvay Congress in Brussels; repr. in CPAE Vol. 3, Doc. 27.

Einstein, Albert and Adriaan Daniël Fokker (1914). "Die Nordströmsche Gravitationstheorie vom Standpunkt des absoluten Differentialkalküls." Annalen der Physik. $4^{\text {th ser. } 44,321-328 .}$

Einstein, Albert and Jakob Grommer (1923). "Beweis der Nichtexistenz eines überall regulären zentrisch symmetrischen Feldes nach der Feldtheorie von Kaluza." Scripta Universitatis atque Bibliothecae Hierosolymitanarum: Mathematica et Physica 1, 1-5. Repr. in CPAE Vol. 13, Doc. 12.

Einstein, Albert and Leopold Infeld (1938). The Evolution of Physics. The Growth of Ideas from Early Concepts to Relativity and Quanta. New York: Simon/Schuster, 1938.

Einstein, Albert and Jakob Johann Laub (1908a). "Die im elektromagnetischen Felde auf ruhende Körper ausgeübten ponderomotorischen

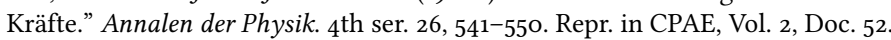

- (1908b). "Elektromagnetische Grundgleichungen für bewegte Körper." Annalen der Physik. 4th ser. 26, 532-540. Repr. in CPAE Vol. 2, Doc. 51.

- (1909). "Bemerkungen zu unserer Arbeit: Elektromagnetische Grundgleichungen für bewegte Körper.” Annalen der Physik. 4th ser. 28, 445-447. Repr. in CPAE Vol. 2, Doc. 54.

Epstein, Paul S. (1911). "Uber relativistische Statik." Annalen der Physik. 4th ser. 341, 779-795.

Felline, Laura (2011). "Scientific Explanation between Principle and Constructive Theories.” Philosophy of Science 78, 989-10oo.

Fermi, Enrico (1921). "Sulla dinamica di un sistema rigido di cariche elettriche in moto translatorio." Nuovo Cimento $22,199-207$.

- (1922). "Über einen Widerspruch zwischen der elektrodynamischen und der relativistischen Theorie der elektromagnetischen Masse." Physikalische Zeitschrift 22, 340-344.

Flores, Francisco J. (1999). "Einstein's Theory of Theories and Types of Theoretical Explanation." International Studies in the Philosophy of Science 13, 123-134.

Fölsing, Albrecht (1993). Albert Einstein. Eine Biographie. Frankfurt: Suhrkamp Verlag, 1993.

Föppl, Ludwig and Percy Danlell (1913). "Zur Kinematik des Bornschen starren Körpers.” Nachrichten von der Gesellschaft der Wissenschaften zu Göttingen 4, 519-529.

Frank, Philipp (1908). "Das Relativitätsprinzip der Mechanik und die Gleichungen für die elektromagnetischen Vorgänge in bewegten Körpern.” Annalen der Physik 332, 897-902.

- (1909). "Die Stellung des Relativitätsprinzips im System der Mechanik und der Elektrodynamik." SBKAWW 118, 373-446.

Frisch, Mathias (2005). "Mechanisms, Principles, and Lorentz's Cautious Realism." Studies in History and Philosophy of Science. Part B: Studies in History and Philosophy of Modern Physics 36, 659-679. 
Frisch, Mathias (2011). "Principle or constructive relativity." Studies in History and Philosophy of Science. Part B: Studies in History and Philosophy of Modern Physics 42, 176-183.

Gibbs, Josiah Willard (1875-1878). "On the Equilibrium of Heterogeneous Substances." Transactions of the Connecticut Academy of Arts and Sciences $3,108-248,343-524$.

Giovanelli, Marco (2018a). “Physics Is a Kind of Metaphysics’. Émile Meyerson and Einstein’s Late Rationalistic Realism.” European fournal for Philosophy of Science 8, 783-829.

- (2018b). “'What is Truth?' Einstein on Rods and Clocks in Relativity Theory.” Habilitation. Universität Tübingen, 2018.

Goenner, Hubert F. M. (2010). "Max Plancks Beiträge zur speziellen Relativitätstheorie." In: Max Planck und die moderne Physik. Ed. by Dieter Hoffmann. Berlin/Heidelberg: Springer, 2010, 149-166.

Goldberg, Stanley (1976). "Max Planck's Philosophy of Nature and His Elaboration of the Special Theory of Relativity." Historical Studies in the Physical Sciences 7, 125-160.

Hecke, Erich (1913). Elektronentheorie. Vorlesungsausarbeitung. Göttingen, 1913.

Hecke, Erich and Wilhelm Behrens (1912). "Ueber die geradlinige Bewegung des Bornschen starren Elektrons." Nachrichten von der Gesellschaft der Wissenschaften zu Göttingen, Mathematisch-physikalische Klasse, 849-86o.

Helmholtz, Hermann von (1887). "Ueber die physikalische Bedeutung des Princips der kleinsten Wirkung." fournal für die reine und angewandte Mathematik 100, 137-166.

Hentschel, Klaus (1992). "Einstein's Attitude Towards Experiments. Testing Relativity Theory 1907-1927." Studies in History and Philosophy of Science 23, 593-624.

Herglotz, Gustav (1903). "Zur Elektronentheorie." Nachrichten von der Königlichen Gesellschaft der Wissenschaften zu Göttingen, Mathematischphysikalische Klasse, 357-382.

- (1910). "Über den vom Standpunkt des Relativitätsprinzips aus als 'starr' zu bezeichnenden Körper.” Annalen der Physik. 4th ser. 31, 393-415.

- (1911). "Über die Mechanik des deformierbaren Körpers vom Standpunkte der Relativitätstheorie." Annalen der Physik. 4th ser. 36, 493-533.

Hertz, Paul (1904). "Untersuchungen über unstetige Bewegungen eines Elektrons.” PhD thesis. Universität Göttingen, 1904.

Hilbert, David (1915). "Grundlagen der Physik." Erste Mitteilung, vorgelegt in der Sitzung vom 20. November 1915. Nachrichten von der Königlichen Gesellschaft der Wissenschaften zu Göttingen, Mathematisch-physikalische Klasse, 395-407. Repr. in Hilbert, 2009 ch. I, 28-46.

- (2009). David Hilbert's Lectures on the Foundations of Physics 1915-1927. Ed. by Tilman Sauer and Ulrich Majer. Berlin/Heidelberg: Springer, 2009.

Hon, Giora (1995). "Is the Identification of Experimental Error Contextually Dependent? The Case of Kaufmann's Experiment and Its Varied Reception." In: 1995, 170-223.

Howard, Don (2005). Einstein's Philosophy of Science. In: Stanford Encyclopedia of Philosophy. Ed. by Edward N. Zalta. Spring. 2005.

- (2007). “'And I Shall Not Mingle Conjectures with Certainties'. On the Intellectual Background to Einstein’s Distinction between Principle Theories and Constructive Theories." 2007. Manuscript.

Hupka, Erich. (1909). "Beitrag Zur Kenntnis der trägen Masse bewegter Elektronen.” Annalen der Physik. 4th ser. 30, 169-204.

Ignatowski, Vladimir Sergeyevitch (1910a). "Der starre Körper und das Relativitätsprinzip." Annalen der Physik. 4th ser. 34, 607-63o.

- (1910b). "Einige allgemeine Bemerkungen zum Relativitätsprinzip." Physikalische Zeitschrift 11, 972-976.

- (1911a). "Zur Elastizitätstheorie vom Standpunkt des Relativitätsprinzips." Physikalische Zeitschrift 12, 164-169.

- (1911b). "Zur Hydrodynamik vom Standpunkte des Relativitätsprinzips." Physikalische Zeitschrift 12, 441-442.

Illy, Jósef (1979). "Albert Einstein in Prague." Isis 70, 76-84.

Illy, József (1981). "Revolutions in a Revolution." Studies in History and Philosophy of Science Part A 12, 175-210.

Janssen, Michel (1995). "A Comparison between Lorentz's Ether Theory and Special Relativity in the Light of the Experiments of Trouton and Noble.” PhD thesis. University of Pittsburgh, 1995.

- (2003). "The Trouton Experiment, $E=M c^{2}$, and a Slice of Minkowski Space-time." In: Revisiting the Foundations of Relativistic Physics. Festschrift in Honor of fohn Stachel. Ed. by Jürgen Renn et al. Dordrecht: Springer, 2003, 27-54.

- (2006). "Electromagnetic Models of the Electron and the Transition from Classical to Relativistic Mechanics." In: Interactions. Mathematics, Physics and Philosophy, 1860-1930. Ed. by Vincent F. Hendricks et al. Dordrecht: Springer, 2006, 64-134.

- (2016). Einstein from field equations to gravitational waves (1912-1918). 32nd Boulder Conference on the History and Philosophy of Science Gravity: Its History and Philosophy - University of Colorado at Boulder - October 28-30, 2016. 2016.

- (2019). "Arches and Scaffolds. Bridging Continuity and Discontinuity in Theory Change." In: 2019.

Janssen, Michel and Jürgen Renn (2015). "History: Einstein Was No Lone Genius." Nature 527, 298-300.

Jeans, James Hopwood (1908). "Zur Strahlungstheorie." Physikalische Zeitschrift 9, 853-855.

Jüttner, Ferencz (1911a). “Das Maxwellsche Gesetz der Geschwindigkeitsverteilung in der Relativtheorie.” Annalen Der Physik 339, 856-882.

- (1911b). "Die Dynamik eines bewegten Gases in der Relativtheorie." Annalen Der Physik 340, 145-161.

Kaluza, Theodor (1921). "Zum Unitätsproblem der Physik." Sitzungsberichte der Preußischen Akademie der Wissenschaften, 966-972.

Kaufmann, Walter (1901). "Die magnetische und elektrische Ablenkbarkeit der Becquerelstrahlen." Göttinger Nachrichten 2, 143-168.

- (1902a). "Die elektromagnetische Masse des Elektrons." Physikalische Zeitschrift 4, 54-57.

- (1902b). "Über die elektromagnetische Masse des Elektrons." Göttinger Nachrichten 5, 291-296.

- (1905). "Über die Konstitution des Elektrons." Gesammtsitzung vom 16. November 1905. Sitzungsberichte der Preußischen Akademie der Wissenschaften, 949-956.

- (1906). "Uber die Konstitution des Elektrons." Annalen der Physik. 4th ser. 18, 487-553.

Kervaire, Michel A. and André Mercier, eds. (1956). Fünfzig fahre Relativitätstheorie - Cinquantenaire de la théorie de la relativité - Jubilee of relativity theory. Basel: Birkhäuser, 1956

Klein, Martin J. (1967). “Thermodynamics in Einstein's Thought.” Science 157, 509-516.

- (1970). Paul Ehrenfest. The Making of a Theoretical Physicist. Vol. 1. Amsterdam: North Holland, 1970.

- (1980). "No firm Foundation. Einstein and the Early Quantum Theory." In: Some Strangeness in the Proportion. A Centennial Symposium to Celebrate the Achievements of Albert Einstein. Ed. by Harry Woolf. London et al.: Addison-Wesley, 1980, 161-185.

Lamla, Ernst (1912a). "Über die Hydrodynamik des Relativitätsprinzips." PhD thesis. Friedrich-Wilhelms-Universität zu Berlin, 1912.

- (1912b). "Über die Hydrodynamik des Relativitätsprinzips." Annalen der Physik 37, 772-796.

Lanczos, Cornelius (1955). "Albert Einstein and the Theory of Relativity." Il Nuovo Cimento 10, 1193-1220.

- (1959). "Albert Einstein and the Role of Theory in Contemporary Physics." American Scientist 47, 41-59. Repr. in Lanczos, 1998 Vol. 2 , $1764-2-1791$.

- (1965). Albert Einstein and the cosmic world order. Wiley: New York, 1965.

- (1998). Collected Published Papers with Commentaries. Ed. by William R. Davis et al. 6 vols. Raleigh: College of Physical/Mathematical Sciences, North Carolina State University, 1998. 
Lange, Marc (2007). "Laws and Meta-laws of Nature. Conservation Laws and Symmetries." Studies in History and Philosophy of Science. Part B: Studies in History and Philosophy of Modern Physics 38, 457-481.

- (2014). “Did Einstein Really Believe That Principle Theories Are Explanatorily Powerless?” Perspectives on Science 22, 449-463.

Laub, Jakob Johann (1907). "Zur Optik der bewegten Körper I." Annalen der Physik. 4th ser. 22, 738-744.

- (1908). "Zur Optik der bewegten Körper II." Annalen der Physik. $4^{\text {th ser. } 24,175-184 .}$

Laue, Max (1907). "Die Mitführung des Lichtes durch bewegte Körper nach dem Relativitätsprinzip." Annalen der Physik. 4th ser. 22, 538-547. Repr. in Laue, 1961 Vol. 1, Doc. 6.

- (1911a). "Bemerkungen zum Hebelgesetz in der Relativitätstheorie.” Physikalische Zeitschrift 12, 1008-1010. Repr. in Laue, 1961 Vol. 1, Doc. 12 .

- (1911b). Das Relativitätsprinzip. Braunschweig: Vieweg, 1911.

- (1911c). "Zur Diskussion über den starren Körper in der Relativitätstheorie.” Physikalische Zeitschrift 12, 85-87. Repr. in Laue, 1961 Vol. 1, Doc. 9.

- (1911d). "Zur Dynamik der Relativitätstheorie." Annalen der Physik. 4th ser. 340, 524-542. Repr. in Laue, 1961. Vol. 1, Doc. 10.

- (1913). "Das Relativitätsprinzip.” Jahrbücher der Philosophie 1, 99-128. Repr. in Laue, 1961. Vol. 1, Doc. 1.

Laue, Max von (1920). "Theoretisches über neuere optische Beobachtungen zur Relativitätstheorie.” Physikalische Zeitschrift 21, 659-662. Repr. in Laue, 1961 Vol. 1, Doc. 37.

- (1952). Die Relativitätstheorie. Bd. 1: Die spezielle Relativitätstheorie. Vieweg: Braunschweig, 1952.

- (1961). Gesammelte Schriften und Vorträge. 3 vols. Braunschweig: Vieweg, 1961.

Lenard, Philipp (1911). Über Äther und Materie. Vortrag, gehalten in der Gesamtsitzung der Heidelberger Akademie der Wissenschaften am 4. Juni 1910. Heidelberg: Winter, 1911.

- (1918). "Über Relativitätsprinzip, Äther, Gtavitation.” Jahrbuch der Radioaktivität und Elektronik 15, 117-136. Über Relativitätsprinzip, Äther, Gravitation. Leipzig: Hirzel, 1918.

Levi-Civita, Tullio (1908). Sui campi electromagnetici puri. Venezia: Ferreri, 1908.

- (1909). "Sulle azioni meccaniche dovute ad un flusso filiforme di elettricità.” Rendiconti della Reale Accademia dei Lincei 5, 41-50.

- (1910). "Über Lorentz-Einsteinsche starre Bewegungen.” Annalen der Physik. 4th ser. 31, 236-240.

Liu, Chuang (1991). "Relativistic Thermodynamics. Its History and Foundations.” PhD thesis. University of Pittsburgh, 1991.

- (1997). Planck and the Special Theory of Relativity. Proceedings of a Conference Commemorating a Century of Physics, Berlin, 22 - 24 March 1995 - Università degli Studi di Pavia. In: The Emergence of Modern Physics. Ed. by Roger H. Stuwer, Fabio Bevilacqua, and Dieter Hoffmann. Pavia: La Goliardica Pavese, 1997, 287-296.

Lorentz, Hendrik Antoon (1903-1904). "Electromagnetic Phenomena in a System Moving with Any Velocity Smaller Than That of Light." Verhandelingen der Koninklijke Akademie van Wetenschappen te Amsterdam 6, 809-831.

- (1904). "Weiterbildung der Maxwellschen Theorie. Elektronentheorie." In: Encyklopädie der mathematischen Wissenschaften. Ed. by Akademie der Wissenschaften in Göttingen, Sächsisch, Akademie der Wissenschaften zu Leipzig, Bayerische Akademie der Wissenschaften and Österreichische Akademie der Wissenschaften. Vol. 5, Physik, part 2. Leipzig: Teubner, 1904, 145-28o.

- (1908). Le partage de l'énergie entre la matiére pondérable et l'éther. Rome: Reale Accademia dei Lincei, 1908. Repr. in Lorentz, 1935-1939. Vol. 7, 317-343. Also pub. in: Il Nuovo Cimento 5, 1908, 5-34; Revue Générale des Sciences 20, 1908, 14-26; Atti del IV Congresso Internazionale dei Matematici. Vol. 1, ed. by Guido Castelnuovo. Roma: Tipografia della Reale Accademia dei Lincei, 1909, $145^{-165 .}$

- (1909). Le partage de l'énergie entre la matiére pondérable et l'éther. (Roma, 6-11 Aprile 1908). In: Atti del IV Congresso Internazionale dei Matematici. Ed. by Guido Castelnuovo. Vol. 1. Roma: Tipografia della Reale Accademia dei Lincei, 1909, 145-165.

- (1910). "Die Hypothese der Lichtquanten." Physikalische Zeitschrift 11, 349-354.

- (1935-1939). Collected Papers. Ed. by Pieter Zeeman and Adriaan Daniël Fokker. 9 vols. The Hague: Nijhoff, 1935-1939.

Maltese, Giulio and Lucia Orlando (1995). "The Definition of Rigidity in the Special Theory of Relativity and the Genesis of the General Theory of Relativity." Studies in History and Philosophy of Science. Part B: Studies in History and Philosophy of Modern Physics 26, 263-306.

Martinez, Alberto A. (2004). "Ritz, Einstein, and the Emission Hypothesis." Physics in Perspective (PIP) 6, 4-28.

McCormmach, Russell (1970a). "Einstein, Lorentz, and the Electron Theory." Historical Studies in the Physical Sciences 2, 41-87.

- (1970b). "H. A. Lorentz and the Electromagnetic View of Nature." Isis 61, 459-497.

Meyerson, Émile (1925). La déduction relativiste. Paris: Payot, 1925.

Mie, Gustav (1912a). “Grundlagen einer Theorie der Materie.” Erste Mitteilung. Annalen der Physik. 4th ser. 37, 511-534.

- (1912b). "Grundlagen einer Theorie der Materie." Zweite Mitteilung. Annalen der Physik. 4th ser. 39, 1-40.

- (1913). "Grundlagen einer Theorie der Materie." Dritte Mitteilung. Annalen der Physik. 4th ser. 40, 1-66.

Miller, Arthur I. (1981). Albert Einstein's Special Theory of Relativity. Emergence (1905) and Early Interpretation (1905-1911). Reading, Mass. et al.: Addison-Wesley, 1981.

- (1986). Frontiers of physics. 190o-1911. Selected Essays with an Original Prologue and Postscript. Boston: Birkhäuser, 1986.

Minkowski, Hermann (1907a). "Das Relativitätsprinzip.” Typescript. Math. Archiv 6o: 3. Niedersächsische Staats- und Universitätsbibliothek. 1907. Pub. post. as Minkowski, 1915

- (1907b). "Die Grundgleichungen für die elektromagnetischen Vorgänge in bewegten Körpern.” Typescript. Math. Archiv 6o: 4. Niedersächsische Staats- und Universitätsbibliothek; 1907. Pub. as Minkowski, 1908

- (1908). "Die Grundgleichungen für die elektromagnetischen Vorgänge in bewegten Körpern.” Nachrichten von der Königlichen Gesellschaft der Wissenschaften zu Göttingen, Mathematisch-physikalische Klasse, 53-111.

- (1909a). "Raum und Zeit." Physikalische Zeitschrift 10, 104-111.

- (1909b). "Raum und Zeit." Jahresberichte der Deutschen Mathematiker-Vereinigung 18, 75-88. Repr. in MGA. Vol. 2, 431-446. Also pub. in: Physikalische Zeitschrift 10, 1909, 104-111.

- (1911). Gesammelte Abhandlungen. Ed. by David Hilbert, Andreas Speiser, and Hermann Weyl. Leipzig: Teubner, 1911.

- (1915). "Das Relativitätsprinzip." Annalen der Physik. 4th ser. 47, 927-938.

Minkowski, Hermann and Max Born (1910). "Eine Ableitung der Grundgleichungen vom Standpunkte der Elektronentheorie." Aus dem Nachlasse bearbeitet von Max Born. Mathematische Annalen 68 (1), 552-564.

Mirimanoff, Dmitry (1909). "Über die Grundgleichungen der Elektrodynamik bewegter Körper von Lorentz und das Prinzip der Relativität." Annalen der Physik. 4th ser. 333, 192-198.

Mosengeil, Kurd von (1906). "Theorie der stationären Strahlung in einem gleichförmig bewegten Hohlraum." PhD thesis. Friedrich-WilhelmsUniversität zu Berlin, 1906

Noether, Fritz (1910). "Zur Kinematik des starren Körpers in der Relativitätstheorie." Annalen der Physik. 4th ser. 31, 919-944.

Nordström, Gunnar (1908). Die Energiegleichung für das elektromagnetische Feld bewegter Körper. Helsingfors, 1908.

- (1909). "Zur Elektrodynamik Minkowskis." Physikalische Zeitschrift 10, 68187. 
Nordström, Gunnar (1910). "Zur elektromagnetischen Mechanik.” Physikalische Zeitschrift 11, 440-445.

- (1911). "Zur Relativitäitsmechanik deformierbar Körper." Physikalische Zeitschrift 12, 854-857.

- (1912). "Relativitätsprinzip und Gravitation.” Physikalische Zeitschrift 13, 1126-1129.

- (1913a). "Träge und schwere Masse in der Relativitätsmechanik." Annalen der Physik 40, 856-878.

- (1913b). "Zur Theorie der Gravitation vom Standpunkt des Relativitätsprinzips.” Annalen der Physik. 4th ser. 42, 533-554

Norton, John D. (1992). "Einstein, Nordström and the Early Demise of Scalar, Lorentz-covariant Theories of Gravitation." Archive for History of Exact Sciences 45, 17-94.

- (2004). "Einstein's Investigations of Galilean Covariant Electrodynamics Prior to 1905." Archive for History of Exact Sciences 59 , 45 -105.

- (2006). "Atoms, Entropy, Quanta. Einstein's Miraculous Argument of 1905." Studies in History and Philosophy of Science. Part B: Studies in History and Philosophy of Modern Physics 37, 71-100.

Ohanian, Hans C. (2008). Einstein's Mistakes. The Human Failings of Genius. New York/London: W. W. Norton \& Company, 2008.

- (2009). "Did Einstein Prove E $=m c^{2}$ ?" Studies in History and Philosophy of Science. Part B: Studies in History and Philosophy of Modern Physics 40, 167-173.

Pais, Abraham (1982). Subtle is the Lord. The Science and the Life of Albert Einstein. New York: Oxford University Press, 1982.

Pauli, Wolfgang (1919). "Zur Theorie der Gravitation und der Elektrizität von Hermann Weyl." Physikalische Zeitschrift 20, 457-467.

- (1959). "Albert Einstein in der Entwicklung der Physik." Physikalische Blätter 15, 241-245.

- (1994). Writings on Physics and Philosophy. Ed. by Charles P. Enz and Karl von Meyenn. Berlin/Heidelberg: Springer, 1994.

Planck, Max (1887). "Über das Princip der Vermehrung der Entropie. Zweite Abhandlung." Annalen der Physik und Chemie. Wiedemann 31, 189-203. Repr. in Planck, 1958b. Vol. 1, Doc. 8.

- (1900a). "Entropie und Temperatur strahlender Wärme." Annalen der Physik. 4 th ser. 1, 719-737.

- (190ob). “Über irreversible Strahlungsvorgänge.” Nach den Sitzungsber. d. k. Akad. d. Wissenech. zu Berlin vom 4. Februar 1897, 8. Juli 1897, 16. December 1897, 7. Juli 1898, 18. Mai 1899 und nach einem auf der 71. Naturf.-Vera. in München gehaltenen Vorträge für die Annalen bearbeitet vom Verfasser. Annalen der Physik. 4th ser. 1, 69-122. Repr. in Planck, 1958b. Vol. 1, Doc. 36.

- (1900c). "Zur Theorie des Gesetzes der Energieverteilung im Normalspectrum." Verhandlungen der Deutschen Physikalischen Gesellschaft 2, 237-245. Repr. in Planck, 1958b. Vol. 1, Doc. 41.

- (1906a). "Das Prinzip der Relativität und die Grundgleichungen der Mechanik.” Verhandlungen der Deutschen Physikalischen Gesellschaft 8, 136-141. Repr. in Planck, 1958b Vol. 2, Doc. 6o.

- (1906b). "Die Kaufmannschen Messungen der Ablenkbarkeit der $\beta$-Strahlen in ihrer Bedeutung für die Dynamik der Elektronen." Vorgetragen in der Sitzung der physikalischen Abteilung der 78. Versammlung Deutscher Naturforscher und Ärzte in Stuttgart am 19 September 1906. Physikalische Zeitschrift 7, 753-761.

- (1906c). "Die Kaufmannschen Messungen der Ablenkbarkeit der $\beta$-Strahlen in ihrer Bedeutung für die Dynamik der Elektronen." Vorgetragen in der Sitzung der physikalischen Abteilung der 78. Versammlung Deutscher Naturforscher und Ärzte in Stuttgart am 19. September 1906. Verhandlungen der Deutschen Physikalischen Gesellschaft 8, 418-432. Repr. in Planck, 1958b Vol. 2, Doc. 61. Also pub. in: Physikalische Zeitschrift 7, 1906, 753-761; Verhandlungen der Gesellschaft Deutscher Naturforscher und Ärzte zu Stuttgart 2, 1906, 61-62.

- (1907a). "Nachtrag zur Besprechung der Kaufmannschen Ablenkungsmessungen." Verhandlungen der Deutschen Physikalischen Gesellschaft 9, 301-305. Repr. in Planck, 1958b. Vol. 2, Doc. 65.

- (1907b). "Zur Dynamik bewegter Systeme." Gesammtsitzung von 13. Juni 1907. Sitzungsberichte der Preußischen Akademie der Wissenschaften, 542-570. Repr. in Planck, 1958b Vol. 2, Doc. 64.

- (1908a). "Bemerkungen zum Prinzip der Aktion und Reaktion in der allgemeinen Dynamik." Physikalische Zeitschrift 9, 828-830. Repr. in Planck, 1958b. Vol. 2, Doc. 67.

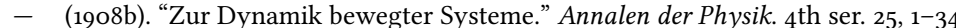

- (1909). "Die Einheit des Physikalischen Weltbildes." Vortrag gehalten am 9.12 .1908 in Leiden. Physikalische Zeitschrift 10, 62-75. Die Einheit des Physikalischen Weltbildes. Vortrag gehalten am 9.12 .1908 in Leiden. Leipzig: Hirzel, 1909.

- (1910a). Acht Vorlesungen über theoretische Physik gehalten an der Columbia university in the City of New York im Frühjahr 19og. Leipzig: Hirzel, 1910

- (1910b). Die Stellung der neueren Physik zur mechanischen Naturanschauung. Vortrag gehalten am 23. September 1910 auf der 82. Versammlung deutscher Naturforscher und Ärzte in Königsberg. Leibzig: Hirzel, 1910. Physikalische Rundblicke. Leipzig: Hirzel, 1922.

- (1910c). "Die Stellung der neueren Physik zur mechanischen Naturanschauung." Vortrag gehalten am 23.9.1910 in Königsberg. PZ 11, 922-932. Repr. in Planck, 1958b Vol. 3, Doc. 1241.

- (1910d). "Gleichförmige Rotation und Lorentz-Kontraktion.” Physikalische Zeitschrift 11, 294. Repr. in Planck, 1958b. Vol. 2, Doc. 72.

- (1910e). "Zur Theorie der Wärmestrahlung.” Annalen der Physik 31, 758-768. Repr. in Planck, 1958a Vol. 2, $237-247$.

- (1958a). Physikalische Abhandlungen und Vortäge. Ed. by Verband Deutscher Physikalischer Gesellschaften and Max-Planck-Gesellschaft zur Förderung der Wissenschaften. 3 vols. Braunschweig: Vieweg, 1958.

- (1958b). Physikalische Abhandlungen und Vorträge. Ed. by Verband Deutscher Physikalischer Gesellschaften and Max-Planck-Gesellschaft zur Förderung der Wissenschaften. 3 vols. Braunschweig: Vieweg, 1958.

Poincaré, Henri (1905). “Sur la dynamique de l'électron.” Séance du Lundi 5 Juin 1905. Comptes Rendus de l'Académie des Sciences 140, $1504-1508$. Repr. in Poincaré, 1928-1956. Vol. 9, 488-491.

- (1906). "Sur la dynamique de l'électron." Adunanza del 23 luglio 1905. Stampato il 14-16 dicembre 1905. Rendiconti del Circolo matematico di Palermo 21, 129-176. Repr. in Poincaré, 1928-1956. Vol. 9, 494-550.

- (1928-1956). Oeuvres de Henri Poincaré. Ed. by Paul Appell and Gaston Darboux. 11 vols. Paris: Gauthier-Villars, $1928-1956$.

Potters, Jan (2019). "Heuristics Versus Norms: On the Relativistic Responses to the Kaufmann Experiments." Studies in History and Philosophy of Science Part B: Studies in History and Philosophy of Modern Physics 66, 69-89.

Pyenson, Lewis (1976). "Einstein's Early Scientific Collaboration.” Historical Studies in the Physical Sciences 7, iv-123.

- (1979). "Physics in the Shadow of Mathematics. The Göttingen Electron-theory Seminar of 1905." Archive for History of Exact Sciences 21, $55^{-89}$.

- (1985). The Young Einstein. The Advent of Relativity. Bristol [and Others: Hilger, 1985

Reichenbächer, Ernst (1917). “Grundzüge zu einer Theorie der Elektrizität und der Gravitation.” Annalen der Physik 357, 134-173.

- (1920). "Inwiefern läßt sich die moderne Gravitationstheorie ohne die Relativität begründen?” Die Naturwissenschaften 8, 10o8-1010.

Renn, Jürgen (2005). "Einstein's Invention of Brownian Motion.” Annalen der Physik 14 (Supplement, 23-37), 23-37.

Rey, Abel (1908). Die Theorie der Physik bei den modernen Physikern. Trans. by Rudolf Eisler. Leipzig: Klinkhardt, 1908.

Ritz, Walther (1908a). "Recherches critiques sur l'Électrodynamique Générale." Annales de Chimie et de Physique 13, 145-275.

- (1908b). "Über die Grundlagen der Elektrodynamik und die Theorie der schwarzen Strahlung." Physikalische Zeitschrift 9, 903-907.

Rowe, David E. (2008). "Max von Laue’s Role in the Relativity Revolution." The Mathematical Intelligencer 30, 54-6o. 
Ryckman, Thomas (2017). Einstein. New York: Routledge, 2017.

Rynasiewicz, Robert and Jürgen Renn (2006). "The Turning Point for Einstein's Annus Mirabilis." Studies in History and Philosophy of Science. Part B: Studies in History and Philosophy of Modern Physics 37, 5-35.

Sauer, Tilman (2008). The Einstein-Varićak Correspondence on Relativistic Rigid Rotation. In: The Eleventh Marcel Grossmann Meeting on recent Developments in Theoretical and Experimental General Relativity, Gravitation and Relativistic field Theories. Ed. by R. T. Jantzen H. Kleinert and R. Ruffini. Singapore Hackensack, N.J.: World Scientific Pub. Co., 20o8, 2453-2455.

Schickore, Jutta (2018). Scientific Discovery. Ed. by Edward N. Zalta. 2018.

Schilpp, Paul Arthur, ed. (1949). Albert Einstein. Philosopher-Scientist. Evanston: The Library of Living Philosophers, 1949.

Schwarzschild, Karl (1903a). "Zur Elektrodynamik. I: Zwei Formen des Princips der Action in der Elektronentheorie." Nachrichten von der Königlichen Gesellschaft der Wissenschaften zu Göttingen, Mathematisch-physikalische Klasse, 126-131.

- (1903b). "Zur Elektrodynamik. II: Die elementare elektrodynamische Kraft." Nachrichten von der Königlichen Gesellschaft der Wissenschaften $z u$ Göttingen, Mathematisch-physikalische Klasse, 132-141.

- (1903c). "Zur Elektrodynamik. III: Ueber die Bewegung des Elektrons.” Nachrichten von der Königlichen Gesellschaft der Wissenschaften zu Göttingen, Mathematisch-physikalische Klasse, 245-278.

Searle, George Frederick Charles (1897). "On the Steady Motion of an Electrified Ellipsoid.” Philosophical Magazine 5, 329-341.

Seelig, Carl (1954). Albert Einstein. Eine dokumentarische Biographie. Zürich: Europa Verlag, 1954.

- (1960). Albert Einstein. Leben und Werk eines Genies unserer Zeit. Vienna: Europa Verlag, 1960.

Seth, Suman (2004). "Quantum Theory and the Electromagnetic World-View." Historical Studies in the Physical and Biological Sciences 35, 67-93.

- (2010). Crafting the Quantum. Arnold Sommerfeld and the Practice of Theory, 1890-1926. Cambridge: MIT Press, 2010.

Shankland, Robert S. (1963). "Conversations with Albert Einstein." American fournal of Physics 31, 47-57.

Sommerfeld, Arnold (1904a). "Zur Elektronentheorie. I. Allgemeine Untersuchung des Feldes eines beliebig bewegten Elektrons.” Nachrichten von der Königlichen Gesellschaft der Wissenschaften zu Göttingen, Mathematisch-physikalische Klasse, 99-130. Repr. in Sommerfeld, 1968. Vol. 2, 39-70.

- (1904b). "Zur Elektronentheorie. II. Grundlagen für eine allgemeine Dynamik des Elektrons.” Nachrichten von der Königlichen Gesellschaft der Wissenschaften zu Göttingen, Mathematisch-physikalische Klasse, 363-439. Repr. in Sommerfeld, 1968. Vol. 2, 71-147.

- (1905). "Zur Elektronentheorie. III. Ueber Lichtgeschwindigkeits- und Ueberichtgeschwindigkeits-Elektronen." Nachrichten von der Königlichen Gesellschaft der Wissenschaften zu Göttingen, Mathematisch-physikalische Klasse, 201-235. Repr. in Sommerfeld, 1968 Vol. 2, 148-182.

- (1906-1907). "Maxwell'sche Theorie u. Elektronen." DMA NL o89 (o28) Winter term. 1906-1907.

- (1907a). "Ein Einwand gegen die Relativtheorie der Elektrodynamik und seine Beseitigung." Physikalische Zeitschrift 8, 841-842.

- (1907b). "Theorie der Strahlung." DMA NL o89 (o28) Summer Term. 1907.

- (1909). "Über die Zusammensetzung der Geschwindigkeiten in der Relativtheorie.” Physikalische Zeitschrift 10, 826-829. Repr. in Sommerfeld, 1968 Vol. 2, 185-188

- (1910a). "Zur Relativitätstheorie. I. Vierdimensionale Vektoralgebra.” Annalen der Physik. 4th ser. 28, 749-776. Repr. in Sommerfeld, 1968. Vol. 2, 189-216.

- (1910b). "Zur Relativitätstheorie. II. Vierdimensionale Vektoranalysis.” Annalen der Physik. 4th ser. 33, 649-689. Repr. in Sommerfeld, 1968 Vol. 2, 217-258.

- (1911). "Das Plancksche Wirkungsquantum und seine allgemeine Bedeutung für die Molekularphysik." Verhandlungen der Gesellschaft Deutscher Naturforscher und Arzte 83, 31-50. Repr. in Sommerfeld, 1968 Vol. 1, Doc. 1. Also pub. in: Physikalische Zeitschrift 12, 1911, 1057-1069.

- (1915). "Review of Born, Dynamik der Kristallgitter [Born, 1915]." Die Naturwissenschaften 50, 669-670.

- (1968). Gesammelte Schriften. Ed. by Fritz Sauter et al. Vol. 4. Braunschweig: Vieweg, 1968.

Stachel, John (200o). "Introduction." In: Einstein. The Formative Years 1879-1909. Ed. by Don Howard and John Stachel. Boston: Univeristy of Notre Dame Press, 2000, 1-22. Repr. in Stachel, 2002 122-139.

- (2002). Einstein from ' $B$ ' to ' $Z$ '. Boston: Birkhäuser, 2002.

Staley, Richard (2008). Einstein's Generation. The Origins of the Relativity Revolution. Chicago: University of Chicago Press, 2008.

Stevens, Syman (2014). "The Dynamical Approach to Relativity as a Form of Regularity Relationalism.” PhD thesis. Exeter College, Oxford University, 2014.

Swann, William Francis Gray (1941). "The Relation of Theory to Experiment in Physics." Reviews of Modern Physics 13, 190-196.

Szarvassi, Arthur (1909). "Die Theorie der elektromagnetischen Erscheinungen in bewegten Körpern und das Energieprinzip." Physikalische Zeitschrift 10, 811-813.

Varićak, Vladimir (1911). "Zum Ehrenfestschen Paradoxon.” Physikalische Zeitschrift 12, 169-170.

Walter, Scott A. (1996). "Hermann Minkowski et la mathématisation 1905-1915." PhD thesis. Université Paris Diderot, 1996.

- (1999). "Minkowski, Mathematicians, and the Mathematical Theory of Relativity. Einstein on the Invariant Content of Space-time Theories." In: The Expanding Worlds of General Relativity. Ed. by Hubert F. M. Goenner et al. Boston: Birkäuser, 1999, 45-86.

- (2007). "Breaking in the 4-Vectors. The Four-Dimensional Movement in Gravitation, 1905-1910." In: The Genesis of General Relativity. Ed. by Jürgen Renn et al. Vol. 3. 4 vols. Dordrecht: Springer, 2007, 193-252.

- (2018). "Ether and Electrons in Relativity Theory." In: 2018.

Weyl, Hermann (1918a). "Gravitation und Elektrizität." Sitzungsberichte der Preußischen Akademie der Wissenschaften, 465-480. Repr. in Weyl, 1968 Vol. 2, Doc. 31.

- (1918b). Raum-Zeit-Materie. Vorlesungen über allgemeine Relativitätstheorie. Berlin: Springer, 1918.

- (1921). Raum-Zeit-Materie. Vorlesungen über allgemeine Relativitätstheorie. $4^{\text {th }}$ ed. Berlin: Springer, 1921

- (1968). Gesammelte Abhandlungen. Ed. by Komaravolu Chandrasekharan. 4 vols. Berlin: Springer, 1968.

Whittaker, Edmund T. (1910). A History of the Theories of Aether and Electricity. From the Age of Descartes to the Close of the Nineteenth Century. London/New York: Longmans, Green/Co., 1910.

- (1953). A History of the Theories of Aether and Electricity. Vol. 2: The modern theories, 1900-1926. London: Nelson, 1953. The first volume appeared as Whittaker, 1910

Wien, Wilhelm (1901). "Ueber die Möglichkeit einer elektromagnetischen Begründung der Mechanik." Aus den Berichten der Société hollandaise des sciences à Harlem. Jubelband für H. A. Lorentz, 11. Dezember 1900. Annalen der Physik. 4th ser. 4, 501-513.

- (1904a). "Erwiderung auf die Kritik des Hrn. M. Abraham." Annalen der Physik. 4th ser. 4, 635-637.

- (1904b). "Zur Elektronentheorie." Physikalische Zeitschrift 5, 393-344 
Wigner, Eugene Paul (1949). "Invariance in Physical Theory." Proceedings of the American Philosophical Society 93 : Theory of Relativity in Contemporary Science. Papers Read at the Celebration of the Seventieth Birthday of Professor Albert Einstein in Princeton, 521-526.

Witte, Hans (1906). "Über den gegen Stand der Frage nach einer mechanischen Erklärung der elektrischen Erscheinungen.” Physikalische Zeitschrift 7, 779-786.

Wolff, Stefan L. (2010). "Das frühe Forschungsprogramm von Max Planck zur Etablierung des zweiten Hauptsatzes der Thermodynamik als allgemeines Naturprinzip." In: Max Planck und die moderne Physik. Ed. by Dieter Hoffmann. Berlin/Heidelberg: Springer, $2010,49-66$.

Wünsch, Daniela (2005). "Einstein, Kaluza, and the fifth Dimension." In: The Universe of General Relativity. Ed. by A. J. Kox and Jean Eisenstaedt. Boston: Birkhäuser, 2005, 277-302. 